\title{
Parton distribution functions and benchmark cross sections at next-to-next-to-leading order
}

\author{
S. Alekhin, ${ }^{1,2, *}$ J. Blümlein, ${ }^{1, \dagger}$ and S. Moch ${ }^{1, \ddagger}$ \\ ${ }^{1}$ Deutsches Elektronensynchrotron DESY, Platanenallee 6, D-15738 Zeuthen, Germany \\ ${ }^{2}$ Institute for High Energy Physics, 142281 Protvino, Moscow region, Russia
}

(Received 22 February 2012; published 10 September 2012)

\begin{abstract}
We present a determination of parton distribution functions (ABM11) and the strong coupling constant $\alpha_{s}$ at next-to-leading order and next-to-next-to-leading order (NNLO) in QCD based on world data for deep-inelastic scattering and fixed-target data for the Drell-Yan process. The analysis is performed in the fixed-flavor number scheme for $n_{f}=3,4,5$ and uses the $\overline{\mathrm{MS}}$ scheme for $\alpha_{s}$ and the heavy-quark masses. At NNLO we obtain the value $\alpha_{s}\left(M_{Z}\right)=0.1134 \pm 0.0011$. The fit results are used to compute benchmark cross sections at hadron colliders to NNLO accuracy and to compare to data from the LHC.
\end{abstract}

DOI: 10.1103/PhysRevD.86.054009

PACS numbers: 12.38.Qk, 12.38.Bx

\section{INTRODUCTION}

Parton distribution functions (PDFs) in the nucleon are an indispensable ingredient of modern collider phenomenology and their study has a long history. In the perturbative approach to the gauge theory of the strong interactions, quantum chromodynamics (QCD), factorization allows for the computation of the hard parton scattering processes as a power series in the strong coupling constant $\alpha_{s}$ and, typically, to leading power $1 / Q^{2}$ dominating for large momentum transfer $Q^{2}$. Predictions for physical cross sections involving initial hadrons, however, do require further nonperturbative information, that is knowledge of the PDFs in the nucleon as well as the value of $\alpha_{s}(Q)$ and of the heavy quark masses. Since PDFs cannot be calculated in perturbative $\mathrm{QCD}$, they need to be extracted from a comparison of theory predictions to available experimental precision data on deep-inelastic scattering (DIS), on the production of lepton-pairs (Drell-Yan process) or jets in hadron collisions or any other suitable hard scattering reaction.

The accuracy of PDF determinations in such analyses has steadily improved over the years, both due to more accurate experimental input and due to refined theory predictions for the hard parton scattering reactions including higher orders in perturbation theory. As of now, complete next-to-next-to-leading order (NNLO) calculations in perturbative QCD form the backbone of this endeavor. These allow for the computation of many important benchmark cross sections, e.g., in the proton-proton collisions at the LHC, with an unprecedented precision. The determination of PDFs to NNLO accuracy in QCD was pioneered more than a decade ago in Ref. [1] and builds in particular on the known corrections for the PDF evolution $[2,3]$ as well as on the hard scattering corrections for DIS [4-10],

\footnotetext{
*sergey.alekhin@ihep.ru

†johannes.bluemlein@desy.de

¥sven-olaf.moch@desy.de
}

and hadronic $W$-and $Z$-gauge boson production, both at an inclusive [11,12] and a differential level [13-15].

Presently, NNLO PDFs have been obtained by a number of different groups. In detail, these are ABKM09 [16,17], HERAPDF1.5 [18,19], JR09 [20,21], MSTW [22], and NN21 [23], while CT10 [24] still remains at next-toleading order (NLO) accuracy only. There exist, of course, differences between these PDF sets. These arise from variations in the choice of the parameters, e.g., the value of $\alpha_{s}\left(M_{Z}\right)$, but also from a different theoretical footing for the data analysis. In the latter case, this comprises for instance, the treatment of the heavy-quark contributions in DIS, the corrections for nuclear effects, the inclusion of higher twist (HT) terms, and so on. The implications for precision predictions at $\mathrm{TeV}$-scale hadron colliders can be profound, though, as benchmark cross sections at NNLO in QCD for the production of $W$ - and Z-gauge bosons or the Higgs boson through gluon-gluon-fusion (ggF) show; see e.g., the recent discussion in Refs. [25-30].

In this article we present the PDF set ABM11, which is an updated version of the PDF analyses of ABKM09 [16] and ABM10 [17] in the 3-, 4-, and 5-flavor scheme at NNLO in QCD. These PDFs are obtained from an analysis of the world DIS data combined with fixed-target data for the Drell-Yan (DY) process and for dimuon production in neutrino-nucleon DIS is given in Sec. II A. In the ABM11 fit we are now using the final version of the DIS inclusive data collected by the HERA experiments in run I [18] together with new data of the H1 Collaboration from the HERA low-energy run [31]. Moreover, our update is based on theoretical improvements. For instance, the treatment of the heavy-quark contributions in DIS now employs the running-mass definition in the $\overline{\mathrm{MS}}$ scheme for the heavy quarks [32].

The strong coupling constant $\alpha_{s}\left(M_{Z}\right)$ or, respectively, the QCD scale $\Lambda_{\mathrm{QCD}}$, is a mandatory parameter to be fitted in DIS analyses of world data. Its correct value is of paramount importance for many processes in DIS and at hadron colliders, in particular for Higgs boson production 
in $\mathrm{ggF}$ [26]. An essential criterion for the selection of additional precision data on top of the world DIS data (e.g., those for the DY process or for hadronic weak-boson and jet production cross sections) in the measurement of the QCD scale is the compatibility of these data sets with respect to the experimental systematics in the different measurements. Furthermore, strictly speaking, combined analyses require a theoretical description at the same perturbative order. Because of these reasons, the combination of different data sets needs great care if performed with the goal of a precision measurement of $\alpha_{s}\left(M_{Z}\right)$. Combinations of a wide range of hard scattering data sets of differing quality, as sometimes used in more global fits, are useful only if they indeed lead to a statistically and systematically improved value of $\alpha_{s}\left(M_{Z}\right)$. Of course, a careful check is always required when new data sets are added. As a result of our new analysis we determine in the ABM11 fit the strong coupling constant at NNLO in the $\overline{\mathrm{MS}}$ scheme and present a detailed discussion of the uncertainties and of the impact of individual experiments, showing the great stability in the obtained value of $\alpha_{s}\left(M_{Z}\right)$.

The paper is organized as follows. In Sec. II we describe the theoretical framework of our analysis. In Sec. II A, in particular, the perturbative QCD input including the framework for heavy-quark DIS. Sections II B and IIC are concerned with a detailed account of the nonperturbative corrections and nuclear corrections, which have already been applied in previous PDF analyses [16,17]. Section III features in detail the data analyzed with an emphasis on the systematic and normalization uncertainties. This comprises data on inclusive DIS from the HERA collider and fixed-target experiments in Sec. III A, on the DY process in Sec. III B, and on dimuon production in neutrino-nucleon DIS in Sec. III C.

The main results of the present work are contained in Sec. IV, where we present all PDF parameters along with illustrations of the shapes of PDFs. The numerous checks include studies of the pulls and the statistical quality for all individual experiments as well as a detailed assessment of the power corrections induced by the higher twist terms. As we work in a scheme with a fixed number $n_{f}$ of light quark flavors, a detailed discussion is also devoted to the generation of heavy-quark PDFs. Our determination of the strong coupling constant $\alpha_{s}$ at NNLO in QCD leads to the value $\alpha_{s}\left(M_{Z}\right)=0.1134 \pm 0.0011$. We show the impact of the individual data sets on $\alpha_{s}\left(M_{Z}\right)$ and compare with the determinations from other PDF fits and other measurements included in the current world average. Finally, Sec. IV is complemented with a comparison of moments of PDFs with recent lattice results.

The consequences of the new PDF set ABM11 on standard candle cross section benchmarks are illustrated in Sec. V. We provide cross section values for $W$ and $Z$ boson production in schemes with $n_{f}=4$ and $n_{f}=5$ flavors, and we address the accuracy of theory predictions for all dominant Higgs boson search channels at the LHC. The PDF uncertainties for top-quark pair production are also illustrated highlighting the combined uncertainty in the gluon PDF, $\alpha_{s}$, and the top-quark mass $m_{t}$. Section V finishes with comments on the issue of hadronic jet production, especially from the Tevatron, and the impact of its data on PDF fits. We conclude in Sec. VI and summarize our approach for the handling of the correlated systematic and normalization uncertainties along with the explicit tables for the covariance matrix of the ABM11 fit in the Appendix.

\section{THEORETICAL FRAMEWORK}

Here, we briefly recall the theoretical basis of our PDF analysis, which is conducted in the so-called fixed-flavor number scheme (FFNS) for $n_{f}$ light (massless) quarks. That is to say, we consider QCD with $n_{f}$ light quarks in the PDF evolution, while heavy (massive) quarks only appear in the final state. As far as QCD perturbation theory is concerned, we specifically focus on aspects relevant to NNLO accuracy. For completeness our treatment of power corrections and also of nuclear corrections as needed e.g., for DIS from fixed-target experiments, is documented in Secs. II B and II C. The latter have already been used in our previous PDF determinations $[16,17]$.

\section{A. Perturbative QCD}

The ability to make quantitative predictions in QCD, which is a strongly coupled gauge theory, rests entirely on its factorization property. A cross section for the production of some final state $X$ from scattering of initial state hadrons can be expressed in lepton-nucleon $(e p)$ DIS as

$$
\begin{aligned}
\sigma_{e p \rightarrow l X}= & \sum_{i} \int d z f_{i}\left(z, \alpha_{s}\left(\mu_{r}\right), \mu_{f}^{2}\right) \\
& \times \hat{\sigma}_{e i \rightarrow X}\left(z, Q^{2}, \alpha_{s}\left(\mu_{r}\right), \mu_{r}^{2}, \mu_{f}^{2}\right),
\end{aligned}
$$

for $l=e, \nu$ and in proton-proton collisions $(p p)$ as

$$
\begin{aligned}
\sigma_{p p \rightarrow X}= & \sum_{i j} \int d z_{1} d z_{2} f_{i}\left(z_{1}, \alpha_{s}\left(\mu_{r}\right), \mu_{f}^{2}\right) f_{j}\left(z_{2}, \alpha_{s}\left(\mu_{r}\right), \mu_{f}^{2}\right) \\
& \times \hat{\sigma}_{i j \rightarrow X}\left(z_{1}, z_{2}, Q^{2}, \alpha_{s}\left(\mu_{r}\right), \mu_{r}^{2}, \mu_{f}^{2}\right),
\end{aligned}
$$

where the PDFs in the nucleon $f_{i}(i=q, \bar{q}, g)$ are the objects of our primary interest. They describe the nucleon momentum fraction $z$ (or $z_{1}, z_{2}$ ) carried by the parton and the sums in Eqs. (2.1) and (2.2) run over all light (anti) quarks and the gluon. The parton cross sections denoted $\hat{\sigma}$ are calculable in perturbation theory in powers of the strong coupling constant $\alpha_{s}$ and describe the hard interactions at short distances of order $\mathcal{O}(1 / Q)$. We have also displayed all implicit and explicit dependence on the renormalization and factorization scales, $\mu_{r}$ and $\mu_{f}$. Throughout our analysis, however, we will identify them, $\mu_{r}=\mu_{f}=\mu$. All dependencies of $\sigma_{e p \rightarrow I X}$ and $\sigma_{p p \rightarrow X}$ on the kinematics 
and, likewise the integration boundaries of the convolutions, have been suppressed in Eqs. (2.1) and (2.2), as these are specific to the observable under consideration.

In standard DIS, the (semi-)inclusive cross section $\sigma_{e p \rightarrow l X}$ in Eq. (2.1) depends on the Bjorken variable $x$, the inelasticity $y$, and on $Q^{2}$, the (spacelike) momentum transfer between the scattered lepton and the nucleon. Moreover, it admits a decomposition in terms of the well-known DIS (unpolarized) structure functions $F_{i}$, $i=1,2,3$. QCD factorization applied to the DIS structure functions implies

$$
\begin{aligned}
a_{k} F_{k}\left(x, Q^{2}\right)= & \sum_{i} \int_{x}^{1} \frac{d z}{z} f_{i}\left(z, \alpha_{s}(\mu), \mu^{2}\right) \\
& \times C_{k, i}\left(\frac{x}{z}, Q^{2}, \alpha_{s}(\mu), \mu^{2}\right),
\end{aligned}
$$

where $a_{1}=2, a_{2}=1 / x, a_{3}=1$, and $C_{k, i}$ denote the Wilson coefficients. $F_{L}=F_{2}-F_{T}$ defines the longitudinal structure function in terms of the transverse structure function $F_{T}=2 x F_{1}$; see also Eq. (3.2) below for the relation including target masses. Equation (2.3) integrated over $x$ gives rise to the standard Mellin moments,

$$
F_{k}\left(N, Q^{2}\right)=\int_{0}^{1} d x x^{N-1} a_{k} F_{i}\left(x, Q^{2}\right) .
$$

These link the theoretical description of DIS to the operator-product expansion (OPE) on the light cone. The OPE allows one to express the DIS structure functions as a product of (Mellin moments of) the Wilson coefficients $C_{k, i}$ and operator matrix elements (OMEs) of leading twist (twist-2). Moreover, it admits a well-defined extension in powers of $1 / Q^{2}$ (twist-4, twist-6, and so on), cf. Sec. II B.

The scale dependence of the PDFs is contained in the well-known evolution equations

$$
\begin{aligned}
\frac{d}{d \ln \mu^{2}}\left(\begin{array}{c}
f_{q_{i}}\left(x, \mu^{2}\right) \\
f_{g}\left(x, \mu^{2}\right)
\end{array}\right)= & \sum_{j} \int_{x}^{1} \frac{d z}{z}\left(\begin{array}{cc}
P_{q_{i} q_{j}}(z) & P_{q_{i} g}(z) \\
P_{g q_{j}}(z) & P_{g g}(z)
\end{array}\right) \\
& \times\left(\begin{array}{c}
f_{q_{j}}\left(x / z, \mu^{2}\right) \\
f_{g}\left(x / z, \mu^{2}\right)
\end{array}\right),
\end{aligned}
$$

at leading twist, which is a system of coupled integrodifferential equations corresponding to the different possible parton splittings. The splitting functions $P_{i j}$ in Eq. (2.5) have been determined at NNLO in Refs. [2,3], which implies knowledge on the first three terms in the powers series in $\alpha_{s}$ (suppressing parton indices),

$$
P=\alpha_{s} \sum_{l=0}^{\infty} \alpha_{s}^{l} P^{(l)} .
$$

The PDFs $f_{i}$ are subject to sum rule constraints due to conservation of the quark number and the momentum in the nucleon, which imply at each order in perturbation theory a vanishing first (second) Mellin moment for specific (combinations of) splitting functions $P_{i j}$ in Eq. (2.6). These sum rule constraints relate the PDF fit parameters used in the parametrizations of the input distributions; see Sec. IV. The accuracy of the numerical solution of the differential Eq. (2.5) up to NNLO was tested by comparison to programs such as QCD-PEGASUS [33] or HOPPET [34].

For the massless DIS structure functions we will be using the following input from perturbative QCD at leading twist:

$$
F_{k}=\sum_{l=0}^{\infty} \alpha_{s}^{l} F_{k}^{(l)}, \quad k=2,3, \quad F_{L}=\alpha_{s} \sum_{l=0}^{\infty} \alpha_{s}^{l} F_{L}^{(l)},
$$

where, again, (NLO) NNLO accuracy is defined by the first (two) three terms in the power series in $\alpha_{s}$, cf. Eq. (2.6). The Wilson coefficients for $F_{2}, F_{3}$ in Eq. (2.7) are known to NNLO from Refs. [4-8], and, actually, even to next-tonext-to-next-to-leading order $\left(\mathrm{N}^{3} \mathrm{LO}\right)$ from Refs. [10,35], and for $F_{L}$ to NNLO from Refs. $[9,10]$. Note that in the latter case the perturbative expansion starts at order $\alpha_{s}$, thus NNLO accuracy for $F_{L}$ actually requires three-loop information, which is numerically not unimportant.

Likewise, for the partonic cross sections of the DY process in Eq. (2.2), i.e., for hadronic $W$ and $Z$ boson production, we use

$$
\hat{\sigma}_{i j \rightarrow W^{ \pm} / Z}=\sum_{l=0}^{\infty} \alpha_{s}^{l} \hat{\sigma}_{i j}^{(l)},
$$

with the NNLO results of Refs. [11-15].

At the level of NNLO accuracy, QCD perturbation theory is expected to provide precise predictions as generally indicated by the numerical size of the radiative corrections at successive higher orders and their pattern of apparent convergence. The residual theoretical uncertainty from the truncation of the perturbative expansion is conventionally estimated by studying the scale stability of the prediction, i.e., by variation of the renormalization and factorization scales $\mu_{r}$ and $\mu_{f}$ in Eqs. (2.1) and (2.2). As stated above, we set $\mu_{r}=\mu_{f}=\mu$ in our analysis, and moreover, identify the scale $\mu$ with the relevant kinematics of the process, e.g., $\mu=Q$ for DIS. Currently no PDF fits with an independent variation of $\mu_{r}$ and $\mu_{f}$ are available and we leave this issue for future studies.

One important aspect is the production of heavy quarks in DIS both for the neutral-current (NC) and the chargedcurrent (CC) exchange. In the former case, pair production of charm quarks accounts for a considerable part of the inclusive DIS cross section measured at HERA, especially at small Bjorken- $x$, while the latter case is needed in the description of neutrino-nucleon DIS. At not too large values of $Q^{2}$, the NC reaction is dominated by the photon-gluon fusion process $\gamma^{*} g \rightarrow c \bar{c} X$, while the CC case proceeds through $W^{*} s \rightarrow c$, so that the perturbative 
expansion of the respective heavy-quark structure functions reads

$$
\begin{aligned}
& F_{k, \mathrm{NC}}^{q}\left(x, Q^{2}, m_{q}^{2}\right)=\alpha_{s} \sum_{l=0}^{\infty} \alpha_{s}^{l} F_{k}^{q,(l)}, \\
& F_{k, \mathrm{CC}}^{q}\left(x, Q^{2}, m_{q}^{2}\right)=\sum_{l=0}^{\infty} \alpha_{s}^{l} F_{k}^{q,(l)},
\end{aligned}
$$

where $k=2,3, L$ and $m_{q}$ is the heavy-quark mass. The heavy-quark Wilson coefficients are known exactly to NLO, both for NC [36] and CC [37,38]. The NNLO results for $F_{k, \mathrm{NC}}^{q}$ are, at present, approximate only and based on the logarithmically enhanced terms near threshold [39-41] (see Ref. [42] for threshold resummation in the CC case). As is well known [43], the heavy-flavor corrections to $F_{2}$ are represented with an accuracy of $O(1 \%)$ and better for $Q^{2} / m_{q}^{2} \gtrsim 10$ by the asymptotic expressions, which do not include the terms proportional to powers of $m_{q}^{2} / Q^{2}$. Under this condition the Wilson coefficients are given by Mellin convolutions of massive OMEs [43-46] and the massless Wilson coefficients [4-10]. Fixed Mellin moments of the heavy-quark OMEs have also been computed at three loops in Ref. [47] and first results for general values of Mellin- $N$ have been calculated in Ref. [48]. Mellin space expressions for the NC and CC Wilson coefficients up to $\mathcal{O}\left(\alpha_{s}^{2}\right)$ are available in Refs. [49,50].

In the current PDF analysis, the bulk of data from DIS experiments can be described in a scheme with $n_{f}=3$ light flavors. At asymptotically large scales $Q \gg m_{c}, m_{b}$ the genuine contributions for heavy charm and bottom quarks in a FFNS with $n_{f}=3$ grow as $\alpha_{s}(Q) \ln \left(Q^{2} / m^{2}\right)$, as the quark masses screen the collinear divergence. The standard PDF evolution equations in Eq. (2.5) resum these logarithms at the expense of matching the effective theories, i.e., QCD with $n_{f}$ and $n_{f}+1$ light flavors. This defines a variable-flavor number scheme (VFNS) and gives rise to the so-called heavy-quark PDFs for charm and bottom quarks in QCD with effectively $n_{f}=4$ and $n_{f}=5$ light flavors. The heavy-quark PDFs are generated from the light flavor PDFs in an $n_{f}=3$-flavor FFNS as convolutions with OMEs; see e.g., Refs. [16,44]. The VFNS requires the matching conditions both for the strong coupling $\alpha_{s}$ and the PDFs (through the corresponding OMEs), which are known to $\mathrm{N}^{3} \mathrm{LO}[51,52]$ for $\alpha_{s}$ and to NNLO for the OMEs $[44,46]$. An extensive discussion of the VFNS implementation has been presented in our previous analysis [16].

The heavy-quark masses in Eq. (2.9) are well defined within a specific renormalization scheme, the most popular ones being the on-shell and the $\overline{\mathrm{MS}}$ scheme. The former uses the so-called pole mass $m_{q}$, defined to coincide with the pole of the heavy-quark propagator at each order in perturbative QCD, and known to have intrinsic theoretical limitations. As a novelty of our analysis, we employ the $\overline{\mathrm{MS}}$ scheme for $m_{q}$, which enters both in the massive OMEs and in the Wilson coefficients and introduces a running-mass $m_{q}(\mu)$ depending on the scale $\mu$ of the hard scattering in complete analogy to the running coupling $\alpha_{s}(\mu)$. As a benefit, predictions for the heavy-quark structure functions in terms of the $\overline{\mathrm{MS}}$ mass display better convergence properties and greater perturbative stability at higher orders [32], thus reducing the inherent theoretical uncertainty.

The Fortran code OPENQCDRAD for the numerical computation of all hard scattering cross sections within the present PDF analysis is publicly available [53]. It comprises, in particular, the theory predictions for the DIS structure functions including the heavy-quark contributions as well as for the hadronic $W$ and $Z$ boson production, and it is capable of computing the benchmark cross sections to NNLO accuracy in QCD in Sec. V.

We neglect all effects due to quantum electrodynamics on the PDF evolution. For reasons of consistency, quantum electrodynamics effects (including a photon PDF, see e.g., the analysis in Ref. [54]) are sometimes needed in computations of cross sections including electroweak corrections at higher orders. Quite generally the effects are small though. The NNLO QCD corrections to the photon's parton structure are known [55,56], and we will address this issue in a future publication.

\section{B. Power corrections}

The leading twist approximation to the QCD improved parton model is valid only at asymptotically large momentum transfers $Q^{2}$, and the factorization underlying Eqs. (2.1) and (2.2) is not sensitive to the finite hadron size effects or, equivalently, to soft hadronic scales like the nucleon mass $M_{N}$. At low momentum transfer comparable to the nucleon mass such hadronic effects cannot be ignored, and the standard factorization ansatz acquires power corrections in $1 / Q^{2}$. In the case of PDF analyses the higher twist terms are especially important for the DIS data since they cover a kinematical range down to $Q^{2} \sim M_{N}^{2}$. The power corrections for the kinematics of DY data used in our fit (cf. Sec. III) are negligible due to the large momentum transfer $Q^{2} \gg M_{N}^{2}$ in this case. Therefore, we do not consider power corrections for the DY process.

In DIS the power corrections arise from kinematic considerations once the hadron mass effects are taken into account, i.e., the so-called target mass correction (TMC). The TMC can be calculated in a straightforward way from the leading twist PDFs within the OPE [57]. In our analysis the TMCs are taken into account in the form of the GeorgiPolitzer prescription [57]. For relevant observables, i.e., the structure function $F_{2}$ and the transverse one $F_{T}$, it reads

$$
\begin{aligned}
F_{T}^{\mathrm{TMC}}\left(x, Q^{2}\right)= & \frac{x^{2}}{\xi^{2} \gamma} F_{T}\left(\xi, Q^{2}\right) \\
& +2 \frac{x^{3} M_{N}^{2}}{Q^{2} \gamma^{2}} \int_{\xi}^{1} \frac{d \xi^{\prime}}{\xi^{\prime 2}} F_{2}\left(\xi^{\prime}, Q^{2}\right),
\end{aligned}
$$


and

$$
\begin{aligned}
F_{2}^{\mathrm{TMC}}\left(x, Q^{2}\right)= & \frac{x^{2}}{\xi^{2} \gamma^{3}} F_{2}\left(\xi, Q^{2}\right) \\
& +6 \frac{x^{3} M_{N}^{2}}{Q^{2} \gamma^{4}} \int_{\xi}^{1} \frac{d \xi^{\prime}}{\xi^{\prime 2}} F_{2}\left(\xi^{\prime}, Q^{2}\right),
\end{aligned}
$$

respectively, which holds up to $\mathcal{O}\left(M_{N}^{2} / Q^{2}\right)$. Here $\xi=2 x /(1+\gamma)$ and $\gamma=\left(1+4 x^{2} M_{N}^{2} / Q^{2}\right)^{1 / 2}$ is the Nachtmann variable [58]. The quantities on the righthand side of Eqs. (2.10) and (2.11) are the leading twist structure functions introduced in Eq. (2.7) above.

Power corrections can also arise dynamically as socalled higher twist terms from correlations of the partons inside the hadron. The twist- 4 terms in the nucleon structure function $F_{2}$ turn out to be non-negligible at large $x$ $[59,60]$. Moreover, the higher twist terms in the longitudinal structure function appear to be necessary for the description of the NMC Collaboration data at moderate $x$ $[27,61]$ and the SLAC data on the structure function $R=\sigma_{L} / \sigma_{T}$ [62], where $\sigma_{L}$ and $\sigma_{T}$ are the absorption cross sections for the longitudinally and the transversely polarized virtual photons, respectively [see also Eq. (3.2)]. The OPE provides the framework for the systematic classification of the higher twist terms referring to local composite operators of twist-4 and higher [63]. Nonetheless the shapes of the higher twist terms are poorly known. Therefore they cannot be accounted for on the same solid theoretical footing as the leading twist contributions discussed in Sec. II A. Furthermore, both, the scaling violations and the Wilson coefficients for the various higher twist contributions have not been computed to the same order in perturbation theory as for the leading twist part.

Basically two strategies exist to address the issue of power corrections in the PDF analysis. The first one imposes kinematical cuts on the data. For DIS, these cuts are performed at high hadronic invariant masses $W^{2}=Q^{2}(1 / x-1)+M_{N}^{2}$ where the nucleon mass is included in the kinematical considerations. In this way, one aims at a data sample with reduced sensitivity to power corrections. Typical values for cuts on $W^{2}$ are of the order of $12 \mathrm{GeV}^{2}$. As a drawback of this procedure one eliminates a rather large fraction of data at low $Q^{2}$ with excellent statistical precision. A more serious concern, however, is due to the generally poor theoretical understanding of those nonperturbative QCD effects beyond leading twist factorization. One simply cannot estimate from first principles the region of $Q^{2}$ (or $W^{2}$ ), where power corrections can be safely neglected. Therefore, the present analysis (following [64]) examines both, TMC and higher twist contributions, in detail in order to control and quantify their impact in the determination of the standard leading twist PDFs.

In practice, higher twist contributions are usually parameterized independently from the leading twist one with some function of $x$, which is typically polynomial in $x$. In our analysis the power corrections are non-negligible for the case of the DIS data and are defined within an entirely phenomenologically motivated ansatz, as follows:

$$
F_{i}^{\mathrm{ht}}\left(x, Q^{2}\right)=F_{i}^{\mathrm{TMC}}\left(x, Q^{2}\right)+\frac{H_{i}^{\tau=4}(x)}{Q^{2}}+\frac{H_{i}^{\tau=6}(x)}{Q^{4}}+\ldots,
$$

where $F_{i}^{\mathrm{TMC}}$ are given by Eqs. (2.10) and (2.11). The coefficients $H_{i}$ are parameterized by a cubic spline with the spline nodes selected at $x=0,0.1,0.3,0.5,0.7,0.9$, and 1 . This choice provides sufficient flexibility of the coefficients $H_{i}$ with respect to the data analyzed and, at the same time, keeps a reasonable number of nodes. The values of $H_{i}(1)$ are fixed at zero due to kinematic constraints. The values of $H_{i}(0)$ are also put to zero in view of the fact that no clear signs of any powerlike terms can be found in the low- $x$ HERA data. The rest of the spline-node values of $H_{i}$ were fitted to the data simultaneously with the PDF parameters and the value of $\alpha_{s}$. We neglect the $Q^{2}$ dependence of the higher twist operators due to the QCD evolution. Therefore, the coefficients $H_{i}$ do not depend on $Q^{2}$. This treatment could be further refined by considering the individual (quasi)partonic OMEs along with their renormalization, i.e., their $Q^{2}$ dependence, which is known for the twist-4 operators to first order in $\alpha_{s}$ [65]. Another complication is the emergence of more Bjorken-like variables $x_{i}$, the number of which grows with increasing twist. Experimental information on the other hand is only available for the variable $x=\sum_{i} x_{i}$ and $Q^{2}$. We leave these aspects for future studies.

With the kinematical cuts imposed on the DIS data in our analysis (cf. Sec. III), the twist-6 terms are irrelevant [66]; therefore, the coefficients $H_{i}^{\tau=6}$ in Eq. (2.12) are washed out. The target dependence of the higher twist parametrization in Eq. (2.12) has been studied in Refs. [67-69]. The isospin asymmetry in $H_{T}$ is poorly constrained by the data used in our fit. It is comparable with zero within the uncertainties [67] and therefore it was put to zero in our analysis. The isospin asymmetry in $\mathrm{H}_{2}$ is also numerically small; however, due to lower uncertainties it cannot be put to zero without deterioration of the fit quality. In summary, we fit three twist- 4 coefficients, for the proton $H_{2}^{\mathrm{p}}$, for the neutron $H_{2}^{\mathrm{n}}$, and for the nucleon $H_{T}^{\mathrm{N}}$, in addition to the leading twist terms. The impact of the power corrections on the DIS neutrino-nucleon dimuon production data used in our fit is marginal [68], as it is on the inclusive charge-current data [18]. Therefore, they are not considered for the case of charged-current structure functions.

\section{Corrections for nuclear effects}

For our analysis we select primarily the data obtained with proton targets. However, in some cases the necessary constraints on PDFs come only from nuclear target data. 
For example, the neutral-current DIS off deuterium targets allows the separation of the large- $x u$-and $d$-quark distributions, which contribute to the DIS structure functions in the form of linear combination weighted with the quark charges. However, the analysis of the deuterium data requires modeling of nuclear effects. They include the Fermi-motion and off-shellness of the nucleons, excess of pions in the nuclear matter, Glauber shadowing, etc.; cf. Refs. $[70,71]$ for reviews. Among them only the Fermimotion effects can be calculated with an uncertainty better or comparable to the uncertainty in the existing experimental data. The Fermi-motion correction is given as a convolution of the free nucleon structure functions with the deuterium wave function, which in turn is constrained by the low-energy electron-nuclei scattering data. The parameterization of the off-shell effect used in our fit was obtained from the analysis of the world data on DIS off heavy nuclear targets and extrapolated to the deuterium target. In this way we assume that the nuclear model suggested in Ref. [70] can be applied to the case of light nuclei, like deuterium. This assumption has been recently confirmed for the case of the ${ }^{3} \mathrm{He}$ and ${ }^{9} \mathrm{Be}$ targets [72]. However, in order to take a conservative estimate of the deuteron correction uncertainty due to the off-shellness, effect we vary its magnitude by $50 \%$. The uncertainty obtained in this way is comparable with one given in Ref. [71]. Other nuclear effects, like shadowing and pion excesses in nuclei considered in Ref. [70], were found numerically negligible for the case of deuterium. Thus our model of the nuclear effects for deuterium is based on the combination of the Fermi-motion and the offshellness effects only. The nuclear corrections depend both on the deuterium wave function and the free nucleon structure functions, while the latter include the target mass corrections and the twist-4 terms; cf. Eq. (2.12). Because of the structure function dependence, the value of the correction is sensitive to the fitted parameters and ideally it should be recalculated iteratively in the fit. However, this approach turns out to be rather time consuming. Therefore, we calculate the deuteron correction once at the beginning with the PDFs and twist-4 terms that were obtained in Ref. [16]. Since the deuteron model employed in our fit is the same as the one of Ref. [16], this approach introduces only a marginal bias into the fit. The nuclear corrections at the representative kinematics of the deuterium data is given in Fig. 1 for the cases of the deuterium wave function obtained with the Paris potential of Ref. [73] used in our analysis and the Bonn potential of Ref. [74]. The difference between these two cases is marginal as compared to the errors in the data. The data on dimuon production in the (anti)neutrino-nucleon scattering, which are used in our analysis in order to constrain the strange sea distribution, were obtained on iron targets. Contrary to the neutralcurrent case, for this data sample a particular shape of the nuclear correction at large $x$ is unimportant since data do not populate the region of $x \gtrsim 0.3$. At small $x$ the neutrino-nucleon DIS nuclear corrections are enhanced due to the parity nonconserving part of the charged current [75]; however, their impact on the strange sea distribution is still smaller than its error [68]. Therefore, in modeling of the dimuon production data we employ the nuclear corrections of Ref. [75] without consideration of their uncertainties. The DY data used in our fit span the moderate- $x$ region. For such kinematics the nuclear effects are quite smaller than the errors in data [76] and they are not considered in our analysis.
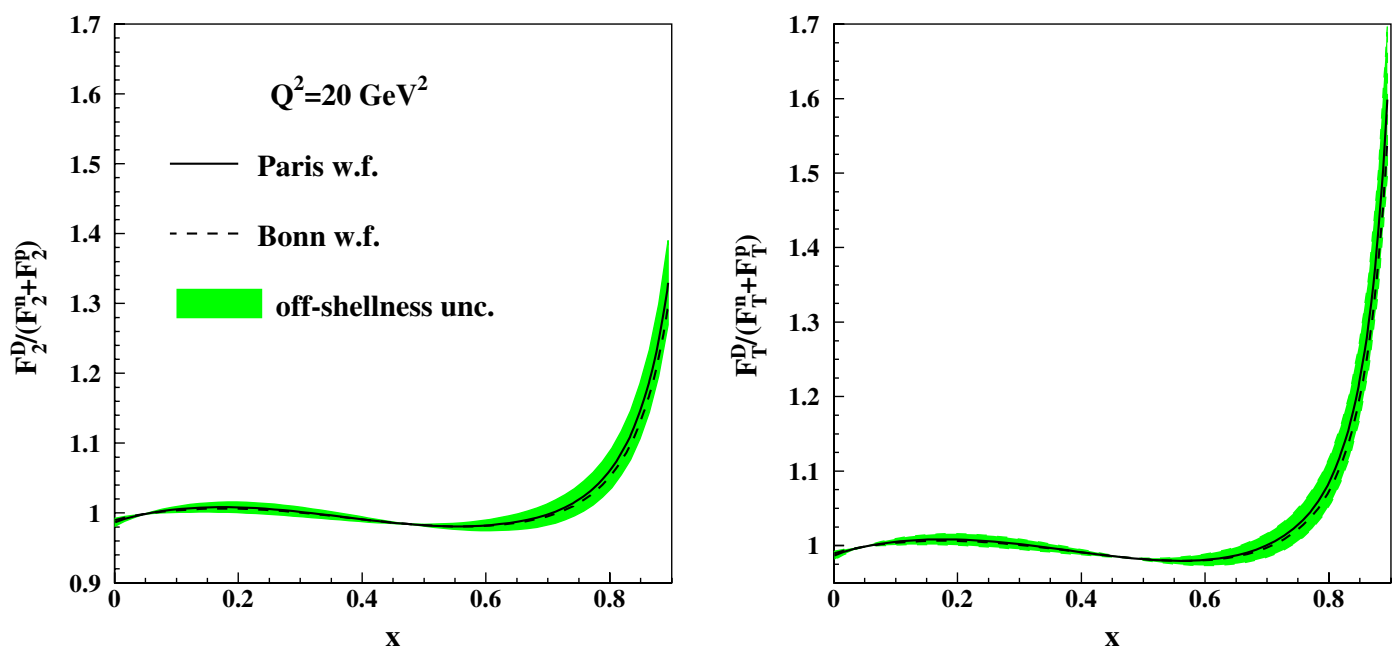

FIG. 1 (color online). The ratio of the deuterium structure function $F_{2}$ (left) and $F_{T}$ (right) with account of the Fermi-motion and offshellness effects of Ref. [70] calculated for the Paris potential of Ref. [73] (solid line) and the Bonn potential of Ref. [74] (dashed line) at the momentum transfer of $20 \mathrm{GeV}^{2}$ to the sum of those for free proton and neutron vs $x$. The shaded area around the solid line gives the uncertainty due to a variation of the off-shell effects by $50 \%$. The calculations are performed at NNLO QCD accuracy using the PDFs and the twist-4 terms obtained in Ref. [16]. 


\section{DATA}

The nucleon PDFs are usually extracted from a combination of hard scattering data, which provides complementary constraints on the different PDF species. A particular choice of which processes to be used in an analysis is commonly driven by the theoretical accuracy of the data modeling and/or the experimental uncertainties in the data. In our fit we employ the data on inclusive DIS, the DY process, and dimuon production in neutrino-nucleon DIS. In combination they allow for a good separation of the quark flavors in a wide range of $x$ and provide good constraints on the gluon distribution at small values of $x$, which are mostly important for the collider phenomenology.

\section{A. Inclusive DIS}

Studies of inclusive DIS date back to the early days of QCD, and since that time a wealth of accurate data has been collected. The first fixed-target DIS experiments at SLAC were followed by data from CERN and Fermilab and then at the electron-proton collider HERA at DESY. The most accurate data of these experiments obtained on the proton and deuterium targets are included in our analysis [31,77-84].

In all cases we employ the data on the inclusive cross section, which is related to the DIS structure functions as follows:

$$
\begin{aligned}
\frac{d^{2} \sigma\left(x, Q^{2}\right)}{d x d Q^{2}}= & \frac{4 \pi \alpha^{2}}{x Q^{4}}\left\{\left(1-y-x y \frac{M_{N}^{2}}{s}\right) F_{2}^{\mathrm{ht}}\left(x, Q^{2}\right)\right. \\
& +\frac{y^{2}}{2}\left(1-\frac{2 m_{l}^{2}}{Q^{2}}\right) F_{T}^{\mathrm{ht}}\left(x, Q^{2}\right) \\
& \left. \pm y\left(1-\frac{y}{2}\right) x F_{3}^{\mathrm{ht}}\left(x, Q^{2}\right)\right\},
\end{aligned}
$$

with the mass of the incident charged-lepton $m_{l}$ and $\pm F_{3}^{\mathrm{ht}}$ corresponding to different polarizations for the case of the charged current. The nucleon structure functions $F_{2}^{\mathrm{ht}}, F_{T}^{\mathrm{ht}}$, and $F_{3}^{\text {ht }}$ are calculated with account of the nuclear correction described in Sec. IIC, if relevant. Note that higher twist contributions to $F_{3}^{\text {ht }}$ are set to zero; cf. Sec. II B. In this way we provide a consistent treatment of the data, contrary to the common procedure in global PDF fits that are based on the data for the structure function $F_{2}$. The structure functions $F_{2}$ and $F_{T}$ in Eq. (3.1) also enter in the ratio of the longitudinally to transversely polarized virtual photon absorption cross sections (see e.g., Ref. [85]),

$$
R\left(x, Q^{2}\right)=\frac{F_{L}\left(x, Q^{2}\right)}{F_{T}\left(x, Q^{2}\right)}=\frac{F_{2}}{F_{T}}\left(1+\frac{4 M_{N}^{2} x^{2}}{Q^{2}}\right)-1 .
$$

In order to avoid contributions from nucleon resonances and the twist- 6 terms, we do not include into the analysis any inclusive DIS data with

$$
Q^{2}<2.5 \mathrm{GeV}^{2}, \quad W<1.8 \mathrm{GeV} .
$$

The kinematics spanned by each DIS data set used in our fit and their systematic uncertainties are described in the following subsections. The normalization uncertainty is a particular case of the systematics. However, it is considered separately since very often the absolute normalization of the DIS experiment is not independently determined. Instead, in such cases it is usually tuned to a selected set of other DIS experiments, which in turn provide the absolute normalization. The wealth of the DIS data used in our fit allows us to extend the basis for this normalization tuning. Therefore, for the experiments lacking an absolute normalization, we consider general normalization factors that are fitted simultaneously with other parameters of our data model. Within this approach we introduce free normalization parameters for the separate early SLAC experiments of Refs. [77-80] and for the NMC data of Ref. [84] at each beam energy. The errors in the normalization factors obtained in our fit are included into the general covariance matrix calculation. In this way we account for the impact of the absolute normalization uncertainty in the data on the PDFs, the higher twist terms, and on the value of $\alpha_{s}$.

Our procedure for the treatment of the DIS data normalization in PDF fits differs substantially from other approaches. For instance, in the MSTW PDF fit [22] free normalization parameters are introduced for all data sets, including even those where the absolute normalization has been determined experimentally. Other PDF fits also commonly employ the NMC data averaged over the beam energies and combined data from the SLAC experiments, rather than the respective individual data sets.

\section{HERA}

In our analysis we use the HERA data on the inclusive neutral-current and the charged-current cross sections [18]. This sample was obtained by a combination of the run I data of the H1 and ZEUS experiments, and includes in particular the data of Refs. [86,87] used earlier in the ABKM09 fit [16]. The HERA data span the region of $Q^{2}$ up to $30000 \mathrm{GeV}^{2}$. However, we impose an additional cut of $Q^{2}<1000 \mathrm{GeV}^{2}$ on the neutral-current sample. This allows one to neglect the $Z$-boson exchange contribution, which is of the order $\sim 1 \%$ at $Q^{2}=1000 \mathrm{GeV}^{2}$. At the same time, the high- $Q^{2}$ part of those data displays only a poor sensitivity to our PDF fit, since the accuracy of those HERA data is $\mathcal{O}(10 \%)$. Therefore, the chosen cut does not distort the fit in any way. The normalization uncertainty in the HERA data of Ref. [18] is 0.5\%, much better than the one in the HERA data of Refs. [86,87]. In particular, due to the improvement in normalization, the new data of Ref. [18] somewhat overshoot the previous H1 data of Ref. [86]. The total number of correlated systematic uncertainties in the HERA data of Ref. [18] is 114, including the uncertainties due to the combination procedure and the general normalization. Many of them are improved as 
compared to the separate experiment samples as a result of cross-calibration in the process of combination.

A complementary set of the inclusive HERA data was obtained by the $\mathrm{H} 1$ Collaboration in the run with a reduced collision energy [31]. These data are particularly sensitive to the structure function $F_{L}$ and thereby to the small- $x$ shape of the gluon distribution. The normalization uncertainty in the low-energy data of Ref. [31] is 3\%. The pointto-point correlated systematic uncertainties come from eight independent sources, and there is also a number of uncorrelated systematic uncertainties in the data.

\section{BCDMS}

The BCDMS data of Refs. [82,83] used in our fit were collected with the CERN muon beam at energies of 100, 120,200 , and $280 \mathrm{GeV}$. Because of the use of both proton and deuterium targets in the same experiment, these data facilitate flavor separation of PDFs at large $x$. The BCDMS absolute normalization was monitored for the beam energy of $200 \mathrm{GeV}$. The general normalization uncertainty in the data due to this monitor is as big as $3 \%$. The absolute normalization of the data obtained at the beam energies of 100,120 , and $280 \mathrm{GeV}$ was calibrated with respect to the case of the beam energy setting of $200 \mathrm{GeV}$. The additional normalization uncertainty due to this calibration ranges from 1 to $1.5 \%$ depending on the beam energy. Other systematic uncertainties in the BCDMS data stem from 5 sources with the most important contributions due to incident and scattered muon energy calibration and the spectrometer resolution. Every source generates a point-to-point correlated uncertainty in the data, while the sources itself are uncorrelated with each other.

\section{3. $N M C$}

The NMC experiment was performed like BCDMS at the CERN muon beam at incident muon energies of 90 , 120, 200, and $280 \mathrm{GeV}$. However, the NMC data span lower values of $x$ and $Q^{2}$ as compared to BCDMS and overlap with the HERA data at the edge of the respective kinematics. We use in our fit the NMC cross section data of Ref. [84] for the proton and deuterium targets. Because of better coverage of the small- $x$ region, those data are also sensitive to the isospin asymmetry in the sea distribution. The absolute normalization for the NMC data of Ref. [84] was determined from tuning for each particular energy setting separately to the BCDMS and SLAC data, which overlap partially with the NMC ones. This tuning in Ref. [84] was based on an empirical data model motivated basically by leading order QCD calculations.

In our analysis, therefore, we fit the NMC normalization factors for each incident beam energy and target simultaneously with the other parameters. In this manner, we ensure consistency with our data model, which in particular includes QCD corrections up to the NNLO; see
TABLE I. NMC normalization factors obtained in our NNLO fit for different incident beam energies and targets.

\begin{tabular}{lcc}
\hline \hline Beam energy $(\mathrm{GeV})$ & Proton & Deuterium \\
\hline 90 & $1.012(12)$ & $0.990(12)$ \\
120 & $1.026(11)$ & $1.005(11)$ \\
200 & $1.034(12)$ & $1.014(11)$ \\
280 & $1.026(11)$ & $1.007(11)$ \\
\hline \hline
\end{tabular}

Ref. [27] for a detailed study of the impact of the NNLO QCD corrections on the interpretation of the NMC data. The normalization factors obtained in the NNLO variant of our fit are given in Table I. In general, they are within the uncertainty of $2 \%$ quoted for the NMC data in Ref. [88]. However, the normalization factors for the proton target are somewhat larger than for the case of deuterium. This is explained by impact of the HERA data of Ref. [18], which slightly overshoot the NMC data in the region of their overlap. The systematic uncertainties in the NMC data are due to the incident and scattered muon energy calibration, the reconstruction efficiency, acceptance, and the electroweak radiative corrections. Some of the systematic uncertainties are correlated for all data, some of them between the proton and deuterium data, and some between beam energies (cf. Ref. [84] for details). In summary, this gives 12 independent sources of systematic uncertainties for the NMC data used in our fit.

\section{SLAC}

The SLAC experiments used in our fit and the number of data points for each experiment after the cut of Eq. (3.3) are listed in Table II. The last and most elaborated in this series is experiment E-140 [81]. In particular, it took advantages of the improved electroweak radiative corrections and the accurate determination of the data absolute normalization, which is as big as $1.8 \%$ for the deuterium sample. Other point-to-point correlated systematic uncertainties are due to background contamination, the spectrometer acceptance, and the electroweak radiative corrections [89]. The rest of systematic error sources for the experiment E-140 are uncorrelated.

The earlier SLAC data used in our fit are collected with various experimental setups and data processing chains. In particular, the electroweak radiative corrections applied to the data differ in details, various methods are used to determine absolute normalization of the data, etc. To overcome this diversity the early SLAC data of Refs. [77-80] were reanalyzed within a uniform approach and the leveled set of the SLAC data was obtained in Ref. [62]. As a part of this leveling the absolute normalization factors for the data of Refs. [77-80] were calibrated with the help of the E-140 data of Ref. [81]. Because of the lack of the E-140 proton data, this calibration is straightforward only for the deuterium case. The proton data normalization tuning was 
TABLE II. List of the SLAC experiments used in our fit (first column: the experiment number; third column: the number of data points (NDP) used in the fit; fourth column: the number of correlated systematic errors (NSE) in the data; fifth column: the normalization factor applied to the data in our fit; sixth column: the normalization factor applied to the data in the reanalysis of Ref. [62]). Note, that the normalization factors of the fifth column apply to the data, which were renormalized in Ref. [62] by the factors given in the sixth column.

\begin{tabular}{lccccc}
\hline \hline Experiment & Target & NDP & NSE & $\begin{array}{c}\text { Normalization } \\
\text { (our fit) }\end{array}$ & $\begin{array}{c}\text { Normalization } \\
\text { (Ref. [62]) }\end{array}$ \\
\hline E-49a [77] & proton & 59 & 3 & $1.022(11)$ & 1.012 \\
& deuterium & 59 & 3 & $0.999(10)$ & 1.001 \\
E-49b [77] & proton & 154 & 3 & $1.028(10)$ & 0.981 \\
& deuterium & 145 & 3 & $1.008(10)$ & 0.981 \\
E-87 [77] & proton & 109 & 3 & $1.032(10)$ & 0.982 \\
& deuterium & 109 & 3 & $1.017(10)$ & 0.986 \\
E-89a [78] & proton & 77 & 4 & 1.0 & 0.989 \\
& deuterium & 71 & 5 & 1.0 & 0.985 \\
E-89b [79] & proton & 90 & 3 & $1.016(10)$ & 0.953 \\
& deuterium & 72 & 3 & $0.996(10)$ & 0.949 \\
E-139 [80] & deuterium & 17 & 3 & $1.014(10)$ & 1.008 \\
E-140 [81] & deuterium & 26 & 5 & 1.0 & 1.0 \\
\hline \hline
\end{tabular}

performed in two steps. First, the normalization of the deuterium data of experiment E-49b was determined with the help of the E-140 deuterium data. Then, the proton data normalization for all other experiments was tuned to the E-49b proton data, assuming equal normalization for the proton and deuterium samples of the E- $49 \mathrm{~b}$ experiment. The data of experiment E-89a are kinematically separated from other SLAC experiments considered. Therefore, their normalization tuning was based on the elastic scattering samples obtained in the experiments E-89a, E-89b, and E-140 (cf. Ref. [62] for the details). In view of the fact that we do not include elastic data in the fit, we keep the normalization of the SLAC experiment E-89a at the value obtained in Ref. [62]. At the same time, in order to take into account the uncertainties in the E-89a data normalization, we add to those data the general normalization uncertainty of $2.8 \%$ and an additional normalization uncertainty of $0.5 \%$ for the case of deuterium, which are quoted in Ref. [62]. The normalization factors for the early SLAC experiments of Refs. [77,79,80] are considered as free parameters of the fit. The SLAC normalization factors for the NNLO variant of our fit are given in Table II in comparison with the ones of Ref. [62], which were obtained with an empirical QCD-motivated model of the data. The deuterium normalization factors obtained in our fit are in a good agreement with the ones of Ref. [62]. For the proton target case our normalization factors are somewhat bigger, in particular, due to a wider set of data being used for the normalization tuning in our case.

\section{B. Drell-Yan process}

The data for the DY process provide a complementary constraint on the PDFs. In particular, they allow one to separate the sea and the valence quark distributions in combination with the DIS data. We use for this purpose the data obtained by the fixed-target Fermilab experiments E-605 [90] and E-866 [91].

The experiment E-605 collected proton-copper collisions data at the center-of-mass energy of $38.8 \mathrm{GeV}$ for dimuon invariant masses in the range of $7-17 \mathrm{GeV}$. At this kinematics the DY data are sensitive to the PDFs down to $x \sim 0.03$. The normalization uncertainty in the E-605 data is $15 \%$. However, other systematic uncertainties in the data are not fully documented in Ref. [90]. The point-to-point correlated systematic is estimated as $+10 \%$ for low dimuon masses and $-10 \%$ for higher masses. Because of lacking details in Ref. [90], we assume a linear dependence of this systematic error on the dimuon mass. Additional uncorrelated systematic uncertainties in the E-605 data due to the Monte Carlo acceptance calculation are combined with the statistical ones in quadrature.

The data of the E-866 experiment on the ratio of the proton-proton and proton-deuterium collision cross sections [91] are particularly sensitive to the isospin asymmetry of the sea quark distributions. The absolute normalization uncertainty cancels in this ratio. Other E-866 systematic uncertainties stem from five independent sources with the biggest contributions due to the deuterium composition and the event detection/ reconstruction. The unpublished data on the absolute DY cross sections for the proton and deuterium targets are also available [92]. However, they are in poor agreement with the DIS data (cf. Ref. [93] for a detailed comparison). Therefore, we do not employ the data of Ref. [92] in our fit. Note that in the MSTW PDF fit [22] the E-866 data on the absolute cross sections are shifted upwards by 
$8.7 \%$ in order to bring them into agreement with the other data sets.

\section{Dimuon production in $\nu \boldsymbol{N}$ DIS}

The production of dimuons in neutrino-nucleon collisions provides unique information about the strange sea distribution in the nucleon. One of the muons produced in this reaction may be resulting from the decay of a charmed hadron. Thus, the production of the $c$ quarks in neutrinonucleon collisions is directly related to initial state strange quarks. Therefore, by relying upon a $c$-quark fragmentation model one can determine the (anti)strange sea distribution from the data on dimuon production in an (anti)neutrino beam. The details of the fragmentation model are quite important in this context due to kinematic cuts imposed to suppress a background of muons coming from the light mesons. Herewith the absolute normalization of the model is defined by the semileptonic branching ratio $B_{\mu}$ of the charmed hadrons. The value of $B_{\mu}$ is poorly known due to the uncertainty in the hadronic charm production rate for the neutrino-nucleon interactions. On the other hand, the value of $B_{\mu}$ is also constrained by the dimuon data themselves $[68,94]$. Therefore, for consistency, we fit the value of $B_{\mu}$ simultaneously with the PDF parameters imposing available independent constraints on $B_{\mu}$ coming from emulsion experiments (cf. Ref. [68] for the details).

We use in the fit the dimuon data provided by two Fermilab experiments, CCFR and $\mathrm{NuTeV}$, and corrected for the cut of $5 \mathrm{GeV}$ imposed on the muon decay energy in order to suppress the light-meson background $[95,96]$. The data of the NuTeV experiment were normalized through the use of the inclusive single muon event rates. Therefore, the normalization error in the data is marginal and it is not considered in our fit. Besides, eight independent sources contribute to the point-to-point correlated systematic uncertainties. The neighboring $\mathrm{NuTeV}$ data points are also correlated due to smearing of the kinematic variables. These correlations are not documented in Ref. [95]. Instead the errors in the data are inflated in such a way that the fit of a model to the data with inflated errors is equivalent to the regular fit with account of the data correlations (cf. Ref. [95] for details). The average data error inflation factor is about 1.4. Therefore, the normal value of $\chi^{2}$ for the inflated-error fit is about one half of the NDP. The CCFR data of Ref. [95] were processed similarly to the NuTeV ones. In particular, the errors in the data were also inflated by a factor of about 1.4 in order to take into account the data point correlations. However, only the combined systematic errors in the CCFR data are available. In view of the lack of any detailed information about the systematic error correlations, we employ in our fit the combined systematic errors quoted in Ref. [95], assuming them to be fully point-topoint correlated.

\section{RESULTS}

We are now in a position to present the results of our analysis ABM11 to NLO and NNLO in QCD for $n_{f}=3$ in a FFNS. The PDF sets for $n_{f}=4$ and 5 are then generated by matching as described above, and we will comment on the changes in the PDFs obtained compared to the ABKM09 set [16]. In addition to the fit results and the covariance matrix for the correlations of the fit parameters, we also present the pulls for separate experiments, which reflect the compatibility of these data sets with respect to the experimental systematics. The discussion of the value of the strong coupling obtained in ABM11 is supplemented by a compilation of $\alpha_{s}\left(M_{Z}\right)$ determinations in NLO and NNLO analyses extending previous studies in Ref. [97]. For a valence distribution we also compute the lowest Mellin moment of our PDFs and compare it with the latest available data from lattice simulations.

\section{A. PDF parameters}

In the new analysis the shape of the PDFs has been updated and the number of fit parameters has been slightly enlarged compared to ABKM09 [16]. In detail, we are using the following parametrizations at the starting scale $\mu^{2}=Q_{0}^{2}=9.0 \mathrm{GeV}^{2}$ in the scheme with $n_{f}=3$ flavors:

$$
\begin{gathered}
x q_{v}\left(x, Q_{0}^{2}\right)=\frac{2 \delta_{q u}+\delta_{q d}}{N_{q}^{v}} x^{a_{q}}(1-x)^{b_{q}} x^{P_{q v}(x)}, \\
x u_{s}\left(x, Q_{0}^{2}\right)=x \bar{u}_{s}\left(x, Q_{0}^{2}\right)=A_{u s}(1-x)^{b_{u s}} x^{a_{u s} P_{u s}(x)}, \\
x \Delta\left(x, Q_{0}^{2}\right)=x d_{s}\left(x, Q_{0}^{2}\right)-x u_{s}\left(x, Q_{0}^{2}\right) \\
=A_{\Delta} x^{a_{\Delta}}(1-x)^{b_{\Delta}} x^{P_{\Delta}(x)}, \\
x s\left(x, Q_{0}^{2}\right)=x \bar{s}\left(x, Q_{0}^{2}\right)=A_{s} x^{a_{s}}(1-x)^{b_{s}}, \\
x g\left(x, Q_{0}^{2}\right)=A_{g} x^{a_{g}}(1-x)^{b_{g}} x^{a_{g} P_{g}(x)},
\end{gathered}
$$

where $q=u, d$ and $\delta_{q q^{\prime}}$ denotes the Kronecker function in Eq. (4.1), and the strange quark distribution is taken to be charge symmetric; cf. Ref. [68]. The polynomials $P(x)$ in Eqs. (4.1), (4.2), (4.3), (4.4), and (4.5) are given by

$$
\begin{gathered}
P_{q v}(x)=\gamma_{1, q} x+\gamma_{2, q} x^{2}+\gamma_{3, q} x^{3}, \\
P_{u s}(x)=\left(1+\gamma_{3, u s} \ln x\right)\left(1+\gamma_{1, u s} x+\gamma_{2, u s} x^{2}\right), \\
P_{\Delta}(x)=\gamma_{1, \Delta} x, \\
P_{g}(x)=\gamma_{1, g} x .
\end{gathered}
$$

The new functional form with the additional parameters $\gamma_{3, u}$ and $\gamma_{3, u s}$ provides sufficient flexibility in the small- $x$ $u$-quark distribution with respect to the analyzed data, and 
we have checked that no additional terms are required to improve the quality of the fit. All 24 PDF parameters are given in Table III together with their $1 \sigma$ uncertainties computed from the propagation of the statistical and systematic errors in the data; cf. the Appendix. Note that the normalization parameters for the valence quarks, $N_{q}^{v}$, and gluons, $A_{g}$, are related to the other PDF parameters due to conservation of fermion number and of momentum, respectively.

As in our previous analysis ABKM09 [16], the small- $x$ exponent $a_{\Delta}$ for the difference between the up- and the down-quark sea is fixed to $a_{\Delta}=0.7$ in Eq. (4.3) as an ansatz because of lacking neutron-target data in this region of small values of $x$. This is in agreement with the values obtained for the small- $x$ exponents of the valence quark distributions. The uncertainty on $a_{\Delta}$ is then determined with help of an additional pseudomeasurement of $a_{\Delta}=$ $0.7 \pm 0.3$ added to the data set (and with the error on $a_{\Delta}$ released) in order to quantify the impact on the other parameters of the PDF fit. This provides us with the result given in Table III. The value of the charmed hadron semileptonic branching ratio $B_{\mu}$ obtained from our NNLO fit is $0.0917 \pm 0.0034$. This is in agreement with the earlier determination [68] within the errors.

The three other parameters of our fit to be discussed in detail in Secs. IVB and IVC, are the strong coupling constant $\alpha_{s}$ in the $\overline{\mathrm{MS}}$ scheme and the heavy-quark masses $m_{c}$ and $m_{b}$, which we take in the $\overline{\mathrm{MS}}$ scheme as well. The latter represents a novel feature of our analysis as all previous PDF determinations have always used the pole mass definition for $m_{c}$ and $m_{b}$. As an advantage, we can constrain the central values of both, $m_{c}\left(m_{c}\right)$ and $m_{b}\left(m_{b}\right)$, directly to their particle data group (PDG) results [98] without having to rely on a perturbative scheme transformation between a running $\overline{\mathrm{MS}}$ mass and a pole mass. It is well known that at low scales such as $\mu \simeq m_{c}$ this scheme transformation is poorly convergent in perturbation theory. Thus, in the present analysis, we add the following pseudodata as input:

$$
\begin{aligned}
& m_{c}\left(m_{c}\right)=1.27 \pm 0.08 \mathrm{GeV}, \\
& m_{b}\left(m_{b}\right)=4.19 \pm 0.13 \mathrm{GeV},
\end{aligned}
$$

and, subsequently, release the uncertainty of the quark masses to test its sensitivity to the other PDF parameters. The value for $\alpha_{s}\left(M_{Z}\right)$, on the other hand, is determined entirely from data in the fit, cf. Sec. IV B. The 24 PDF parameters of Table III, $\alpha_{s}, m_{c}$, and $m_{b}$ provide us in our analysis with 27 correlated parameters in total. Their covariance matrix is presented in the Appendix.

It is instructive to study the pulls of the individual data sets included in the fit. This provides a mean of assessing the quality of the fit in detail and allows for an investigation of specific kinematical regions. In Figs. 2-4 we display the detailed dependence of the pulls on the momentum transfer $Q^{2}$ and $x$ for the HERA NC and CC inclusive DIS cross section data of Ref. [18] as well as the low $Q^{2}$ data of Ref. [31] with respect to our NNLO fit. We find overall a very good description of the data, even at the edges of the kinematical region of HERA, i.e., at smallest values of $x$ and largest values of $Q^{2}$. The respective $\chi^{2}$ values for the fit at NLO and NNLO are given in Table IV.

Next, in Figs. 5-7 we show the respective pulls of the BCDMS [82,83], NMC [84], and SLAC [77-81] inclusive DIS cross section data as a function of $x$ and binned in the momentum transfer $Q^{2}$. Again, our fit provides a very good description (see Table IV for the $\chi^{2}$ values), in particular at low $W^{2}$ thanks to the phenomenological ansatz for the structure functions with the higher twist terms of Table V.

In Fig. 8 we plot pulls of the data for the (anti)neutrino induced dimuon production cross section of Ref. [95], which constrains the strange PDF. We give both, the pulls for the $\mathrm{NuTeV}$ and for the CCFR experiment. Finally, in Fig. 9 we display the pulls of DY cross section data of Refs. [90,91], which depends on the muon pair rapidity $Y$ and the invariant mass $M_{\mu \mu}$ of the muon pair. It is obvious from Figs. 8 and 9, and Table IV that we achieve again a very good description in all cases.

The last missing piece of information on the PDF fit concerns the shape of the higher twist terms for the inclusive DIS structure functions introduced in Eq. (2.12). As outlined in Sec. II B, we fit three twist-4 coefficients for a complete description of both, proton and nucleon targets. In detail, the proton $H_{2}^{\mathrm{p}}$, the nonsinglet $H_{2}^{\mathrm{ns}}=H_{2}^{\mathrm{p}}-H_{2}^{\mathrm{n}}$, and the proton $H_{T}^{\mathrm{p}}$ contribute 15 parameters in total, and we assume $H_{T}^{\text {ns }}=0$. The respective coefficients are listed in

TABLE III. Parameters of the PDFs in Eqs. (4.1), (4.2), (4.3), (4.4), and (4.5) and their $1 \sigma$ errors obtained in the scheme with $n_{f}=3$ flavors.

\begin{tabular}{lccccc}
\hline \hline & $a$ & $b$ & $\gamma_{1}$ & $\gamma_{2}$ & $\gamma_{3}$ \\
\hline$u_{v}$ & $0.712 \pm 0.081$ & $3.637 \pm 0.138$ & $0.593 \pm 0.774$ & $-3.607 \pm 0.762$ & $3.718 \pm 1.148$ \\
$d_{v}$ & $0.741 \pm 0.157$ & $5.123 \pm 0.394$ & $1.122 \pm 1.232$ & $-2.984 \pm 1.077$ & \\
$u_{s}$ & $-0.363 \pm 0.035$ & $7.861 \pm 0.433$ & $4.339 \pm 1.790$ & & $0.0280 \pm 0.0036$ \\
$\Delta$ & $0.70 \pm 0.28$ & $11.75 \pm 1.97$ & $-2.57 \pm 3.12$ & $0.0808 \pm 0.0122$ \\
$s$ & $-0.240 \pm 0.055$ & $7.98 \pm 0.65$ & & $0.316 \pm 0.385$ \\
$g$ & $-0.170 \pm 0.012$ & $10.71 \pm 1.43$ & $4.00 \pm 4.21$ & & $0.085 \pm 0.017$ \\
\hline \hline
\end{tabular}




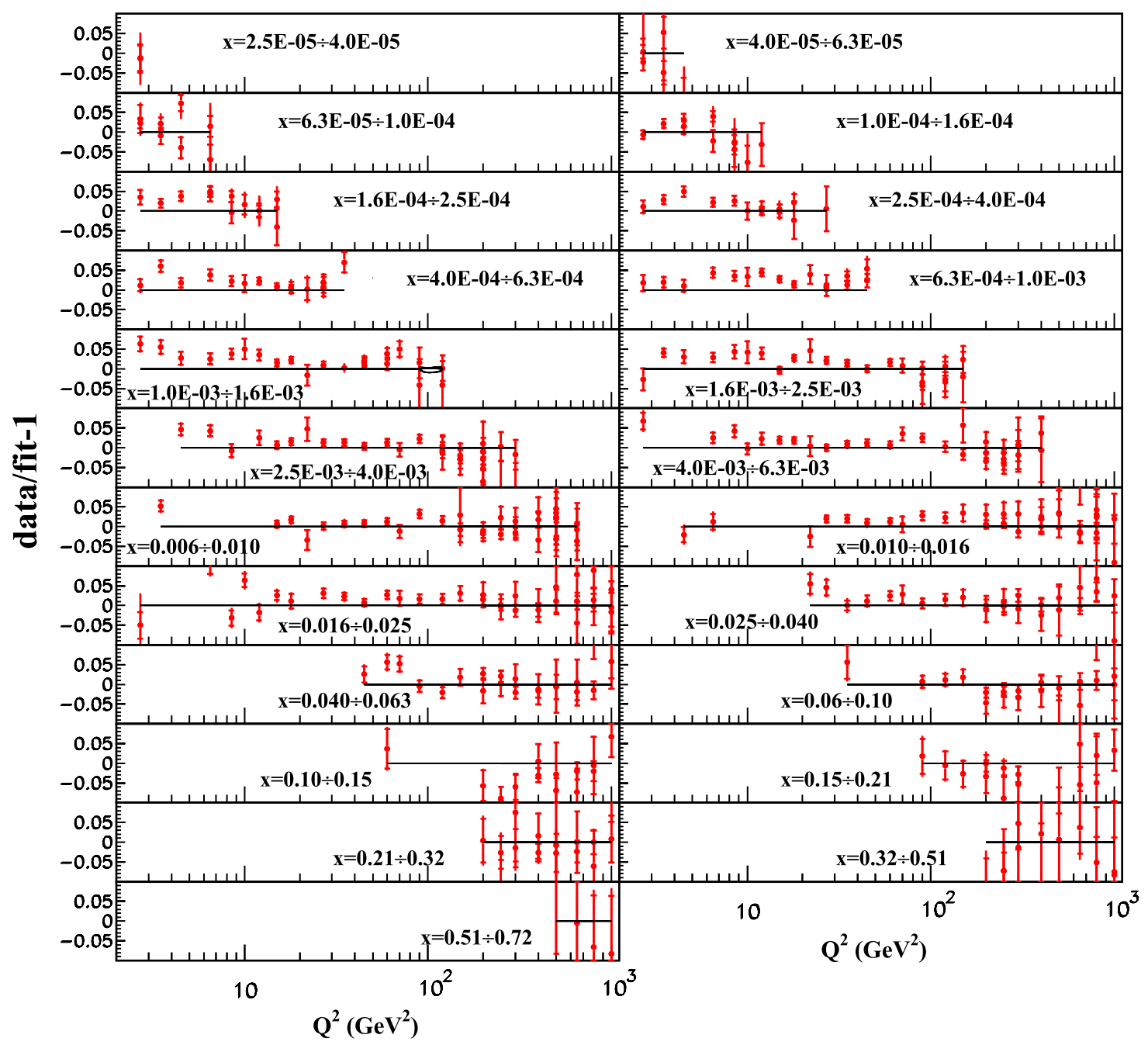

FIG. 2 (color online). Pulls vs momentum transfer $Q^{2}$ for the HERA neutral-current inclusive DIS cross section data of Ref. [18] binned in $x$ with respect to our NNLO fit. Data points with different inelasticity $y$ still may overlap in the plot. Inner bars show statistical errors in data, and outer bars the statistical and systematic errors combined in quadrature.

Table V and shown in Fig. 10, all in units of $\mathrm{GeV}^{-2}$. They are in agreement with the earlier results of Refs. [59,60] up to the parameterization of the HT contribution (compare Eq. (2.12) with Eq. (35) of Ref. [60]). The magnitude of the HT terms reduces from the NLO to the NNLO case, see Fig. 10; however, the change is comparable with the coefficient uncertainties and the NNLO twist-4 coefficients do not still vanish, in line with the results of Ref. [60]. The nonsinglet twist-4 term in $F_{T}$ is comparable to zero within uncertainties; therefore, it was fixed at zero in our analysis as discussed in Sec. II B. The nonsinglet twist- 4 term in $F_{2}$ is negative at $x \leq 0.5$. This is also in line with earlier results [59], again taking into account the difference in the HT parametrizations. The HT terms are mostly important at small hadronic invariant mass $W$. This was confirmed in a comparison of the low- $W$ JLAB data [99] with predictions based on the ABKM09 PDFs with account of the twist- 4 terms, which were extracted in the analysis of Ref. [16] similarly to the present one. However, even with the cut of $W^{2}>12.5 \mathrm{GeV}^{2}$ as commonly imposed in global PDF fits, the HT terms are numerically important for the region of $x \leqq 0.3$, which is not affected by this cut.

From Fig. 11 it is evident that calculations, which are based on our NNLO PDFs but do not include the HT terms, systematically overshoot the SLAC data at $x \lesssim 0.3$ due to the HT terms being negative in this region. The value of $\chi^{2} /$ NDP for the SLAC data at

$$
W^{2}>12.5 \mathrm{GeV}^{2}, \quad Q^{2}>2.5 \mathrm{GeV}^{2}
$$

is $699 / 246$ in this case. This is much worse than the value of $\chi^{2} / \mathrm{NDP}=292 / 246$ obtained in our fit for the same subset of data. We have also performed similar comparisons taking the published 3-flavor NNLO MSTW [22] and NLO NN21 [100] PDFs as an input of our fitting code. The NNLO MSTW and NLO NN21 predictions obtained in this way without accounting for the HT terms and with the cut of Eq. (4.11) imposed lead to a poor description of the SLAC data; cf. Fig. 11. For the case of NN21 PDFs, the 


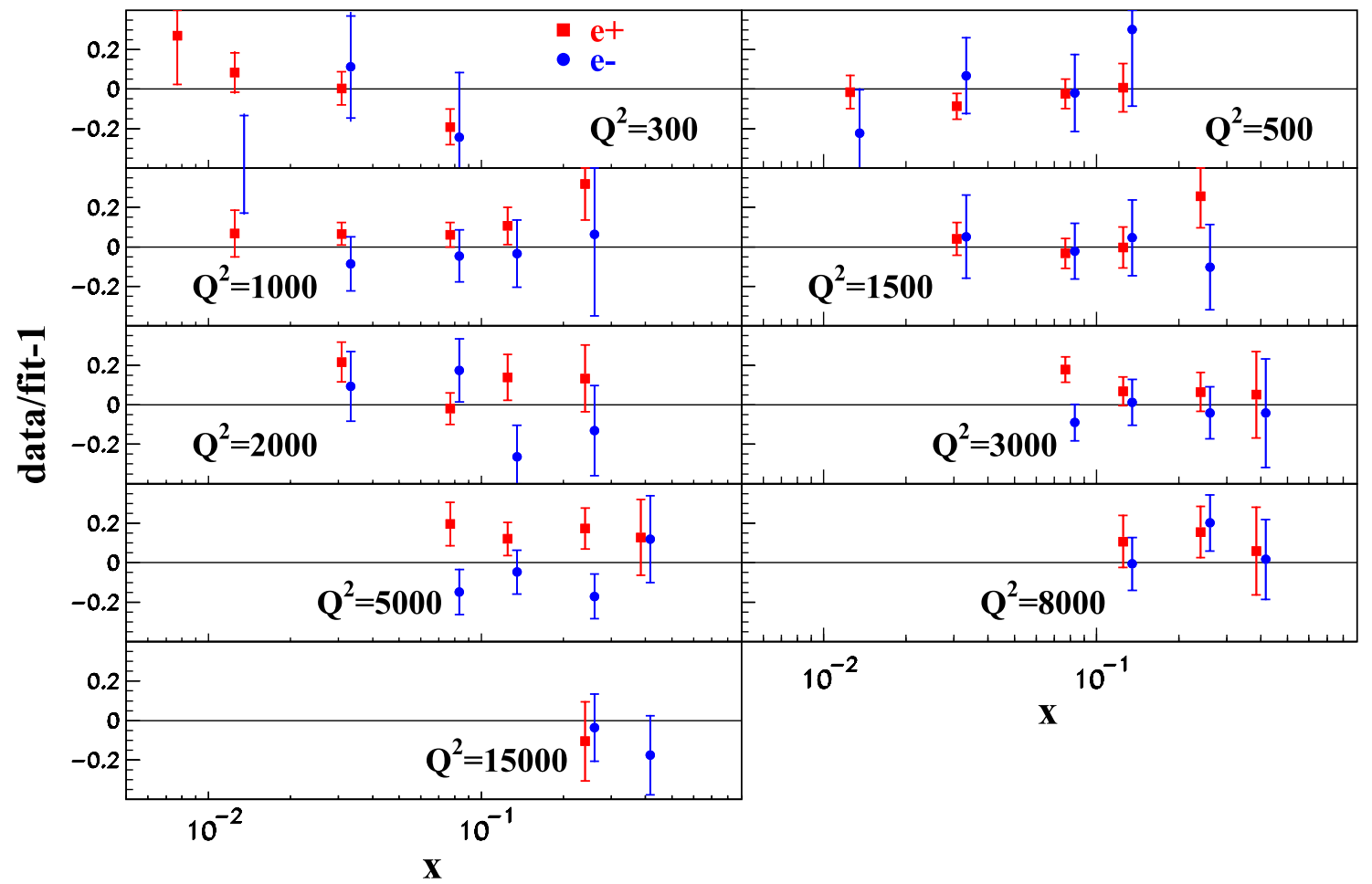

FIG. 3 (color online). Same as Fig. 2 for the pulls of the HERA charged-current inclusive DIS cross section data of Ref. [18] binned in the momentum transfer $Q^{2}$ in units of $\mathrm{GeV}^{2}$ vs $x$ (squares: positron beam; circles: electron beam).

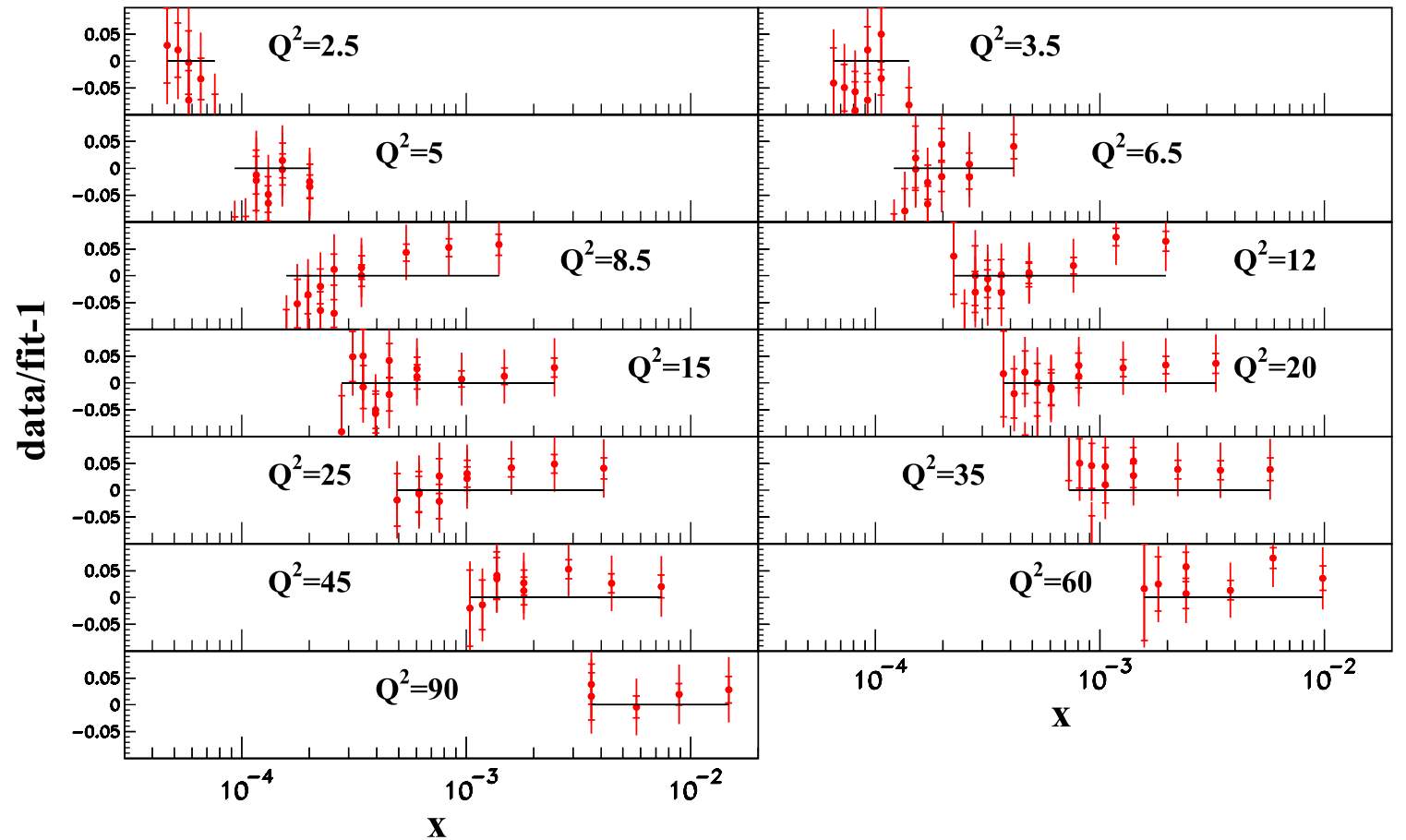

FIG. 4 (color online). Same as Fig. 2 for the pulls of the H1 neutral-current inclusive DIS cross section data of Ref. [31] binned in the momentum transfer $Q^{2}$ in units of $\mathrm{GeV}^{2}$ vs $x$. 
TABLE IV. Value of $\chi^{2}$ obtained in the NNLO and NLO fits for different data sets.

\begin{tabular}{lcccc}
\hline \hline & Experiment & NDP & $\chi^{2}$ (NNLO) & $\chi^{2}$ (NLO) \\
\hline DIS inclusive & H1\&ZEUS [18] & 486 & 537 & 531 \\
& H1 [31] & 130 & 137 & 132 \\
& BCDMS [82,83] & 605 & 705 & 695 \\
& NMC [84] & 490 & 665 & 661 \\
& SLAC-E-49a [77] & 118 & 63 & 63 \\
& SLAC-E-49b [77] & 299 & 357 & 357 \\
& SLAC-E-87 [77] & 218 & 210 & 219 \\
& SLAC-E-89a [78] & 148 & 219 & 215 \\
& SLAC-E-89b [79] & 162 & 133 & 132 \\
& SLAC-E-139 [80] & 17 & 11 & 11 \\
Drell-Yan & SLAC-E-140 [81] & 26 & 28 & 29 \\
& FNAL-E-605 [90] & 119 & 167 & 167 \\
DIS dimuon & FNAL-E-866 [91] & 39 & 52 & 55 \\
Total & NuTeV [95] & 89 & 46 & 49 \\
\hline \hline
\end{tabular}

agreement with the data is very bad, with an offset reaching up to $\sim 10 \%$ at $x \sim 0.15$ and a value of $\chi^{2} / \mathrm{NDP}=$ $518 / 246$. The MSTW value of $\chi^{2} / \mathrm{NDP}=514 / 246$ is also far from ideal in this case, obviously due to the missing HT terms. Also, MSTW does not take into account the target mass corrections. Note, that for the comparison performed without the twist- 4 terms the ABM11 value of $\chi^{2}$ is worse than the ones of MSTW and NN21 since those PDFs are obtained disregarding the HT terms. This also shows that parts of the twist- 4 terms obtained in our fit are effectively absorbed into the MSTW and NN21 PDFs.

The relative contribution of the higher twist terms to the ratio $R=\sigma_{L} / \sigma_{T}$ in Eq. (3.2) is particularly important reaching up to one half at moderate $x$ [101]. The value of $R$ calculated including the NNLO QCD corrections and the twist-4 terms of Table $\mathrm{V}$ is in reasonable agreement with the SLAC data on $R$ [62] and the parameterization of those data $R_{1990}$; cf. Fig. 12 . The latter is based on the empirical combination of the QCD-like terms with the twist-4 and twist-6 terms, pretending to describe the data down to scales $Q^{2} \sim 1 \mathrm{GeV}^{2}$. Because of the twist-6 term, which provides saturation of $R_{1990}$ at small $Q$, the shape of $R_{1990}$ is somewhat different from our calculation, while both agree with the data at $Q^{2}>2 \mathrm{GeV}^{2}$ within the errors. The leading twist NNLO contribution to $R$ undershoots the full calculation by a factor of $1.5-2$, depending on $x$; cf. Fig. 12. The leading twist NLO calculations based on the 3-flavor NN21 PDFs are in a good agreement with our NNLO leading twist term and go by a factor of 1.5-2 lower than the data as well. The leading-twist NNLO calculations for the 3-flavor MSTW PDFs are larger than the NLO NN21 ones and are in better agreement with the data. Note, that this is related to the fact that the data on $R$ of Ref. [62] are included into the MSTW fit allowing for a better description of the SLAC data as compared to the NN21 case. At the same time this leads to an effective absorption of the twist- 4 terms into the fitted PDFs.

The NNLO ABM11 PDFs obtained in the present analysis are compared in Fig. 13 with our earlier ABKM09 PDFs. The biggest change between these two sets is observed for the small- $x$ gluon and sea distributions. First, this change happens since the HERA NC inclusive data [18] used in the present analysis lie by several percent higher than the HERA data of Refs. [86,87] used in the ABKM09 analysis, due to improvements in the monitor calibration. Second, the small- $x$ PDFs are particularly sensitive to the treatment of the heavy-quark electroproduction and, therefore, they change due to the NNLO corrections and the running-mass scheme implemented in the present PDF fit. Other ABM11 PDFs are in agreement with the ABKM09 ones within the uncertainties.

The NNLO PDFs obtained by other groups are compared with the NNLO ABM11 PDFs in Fig. 14. The agreement between the various PDFs is not ideal, a fact that may be explained by the differences in the data sets used to constrain the PDFs, by the factorization scheme employed, by the treatment of the data error correlation, and so on. A detailed clarification of these issues is beyond the scope of the present paper. Therefore, we discuss only the most significant differences, e.g., the gluon distributions at small $x \lessgtr 0.001$, which are quite different for all PDF sets considered in Fig. 14.

To that end, we compare in Fig. 15 the small- $x$ data on $F_{L}$ obtained by the H1 Collaboration [31] with the predictions based on these PDFs. The $F_{L}$ data are quite sensitive to the small- $x$ gluon PDFs. Moreover, in order to provide a consistent comparison, all predictions are taken in the running-mass 3-flavor scheme with the heavy-quark 


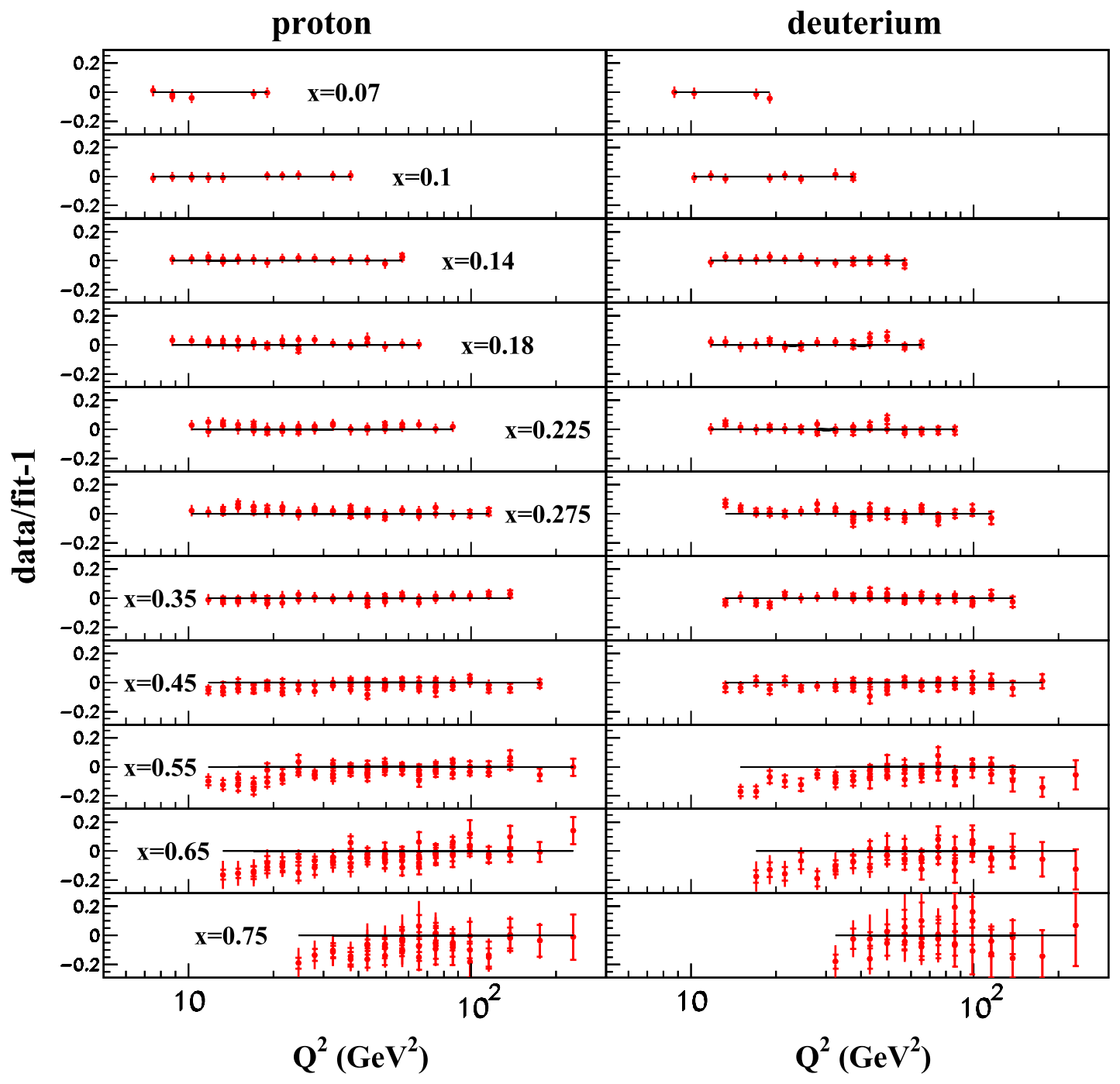

FIG. 5 (color online). Same as Fig. 2 for the pulls of the BCDMS inclusive DIS cross section data of Ref. [82,83] for the proton target (left) and the deuterium target (right).

masses of Eq. (4.10) and with the 3-flavor PDFs. The H1 data on $F_{L}$ are in a good agreement with the NNLO ABM11 predictions. Although these data were not included into the fit of Ref. [20], they are also in a good agreement with the NNLO JR09 predictions. The NNLO MSTW and NLO NN21 predictions, on the other hand, miss the $\mathrm{H} 1$ data. Thus, the latter can be used to consolidate the small- $x$ behavior of the gluon PDFs provided by different groups. Likewise, the SLAC DIS cross section data of Refs. [77-81] can also be of help in consolidating the results of the different PDF fits. As one can see in Fig. 11, the MSTW and NN21 predictions systematically overshoot the SLAC data at $x \sim 0.2$. As we discussed above, this happens due to the omission of the higher twist terms. Once the latter are neglected in the fit, the power corrections are partially absorbed in the leading twist PDFs. Therefore, this discrepancy is evidently also related to the difference of those PDFs with the ABM11 ones at moderate $x$; cf. Fig. 14. On the other hand, the ABM11 large- $x$ gluon distribution goes lower than the NN21 and MSTW ones, because we do not include the Tevatron inclusive jet data into the fit; cf. Ref. [102] and Sec. V D.

Another striking difference in Fig. 12 is related to the strange sea distribution, which is commonly constrained by the data on the dimuon production in the $\nu N$ DIS in all PDF fits considered. Nonetheless, for the NN21 and JR09 sets it goes significantly lower at $x \geqslant 0.02$ than for the MSTW and ABM11 ones. The difference between the NN21 and ABM11 strange sea distributions appears to be due to Eq. (34) of Ref. [100] for the dimuon production cross section, which contains an additional factor of $\left(1+m_{c}^{2} / Q^{2}\right)$ as compared, e.g., to Eq. (3) of Ref. [38] employed in our analysis; cf. also Ref. [50]. At small $Q^{2}$ this factor reaches a numerical value of 2 and the strange sea is suppressed correspondingly in the fit to the data. We have convinced ourselves that with this factor taken into 
proton

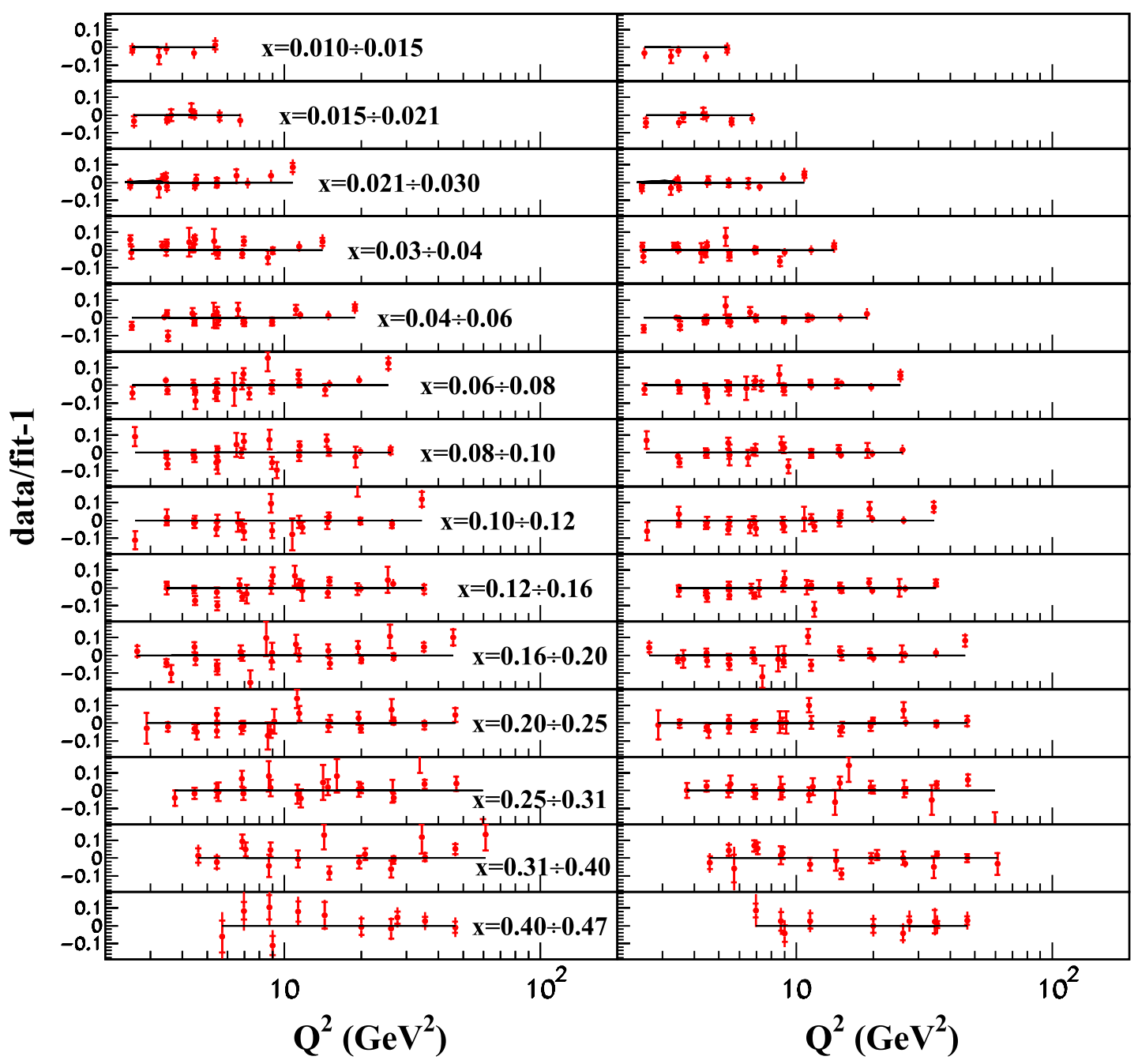

FIG. 6 (color online). Same as Fig. 2 for the pulls of the NMC inclusive DIS cross section data of Ref. [84] for the proton target (left) and the deuterium target (right).

account, the NN21 PDFs deliver a satisfactory description of the CCFR and NuTeV dimuon data. On the other hand, the discrepancy between the results of JR09 and ABM11 in Fig. 14 can directly be traced back to the ansatz $s\left(x, Q_{0}^{2}\right)=$ $\bar{s}\left(x, Q_{0}^{2}\right)=0$ of JR09, i.e., the assumption of vanishing strangeness at the low starting scale of $Q_{0}^{2}<1 \mathrm{GeV}^{2}$ in the dynamical valencelike PDF model of JR09. This is different from ours; cf. Eq. (4.4). Moreover, JR09 has not used the data on dimuon production in neutrino-nucleon collisions in their fit; see Sec. III C.

Finally, the difference in the large- $x$ nonstrange quark distributions appears partly due to the general normalization of the data, which is often a matter of choice in the PDF fits. In order to quantify impact of the choice of the data normalization on the PDFs (cf. Sec. III), we have performed a variant of our NNLO fit with the same normalization factor settings as employed in the MSTW fit [22]. The relative difference between the $u$-and $d$-quark distributions obtained in this variant of the fit and our nominal one is displayed in Fig. 16. Clearly, the impact of the data normalization choice is most pronounced at large $x$, where the trend is different for the cases of $d$ and $u$ quarks. Therefore, the effect is amplified in the ratio $d / u$ which is important for the interpretation of the chargedlepton asymmetry data from hadron colliders; cf. Sec. VA. As shown in Fig. 16, the relative difference in the ratio $d / u$ reaches up to $5 \%$ at $x \sim 0.5$.

\section{B. Strong coupling constant}

For a precision determination of $\alpha_{s}\left(M_{Z}\right)$ the fit of systematically compatible data sets is a necessary prerequisite. Here the individual data sets determine the average in such a way that their individual effect is closely compatible with the central value within the errors. Enhancing the precision from NLO to NNLO, and in some cases to even higher orders, the central values and the values 
proton

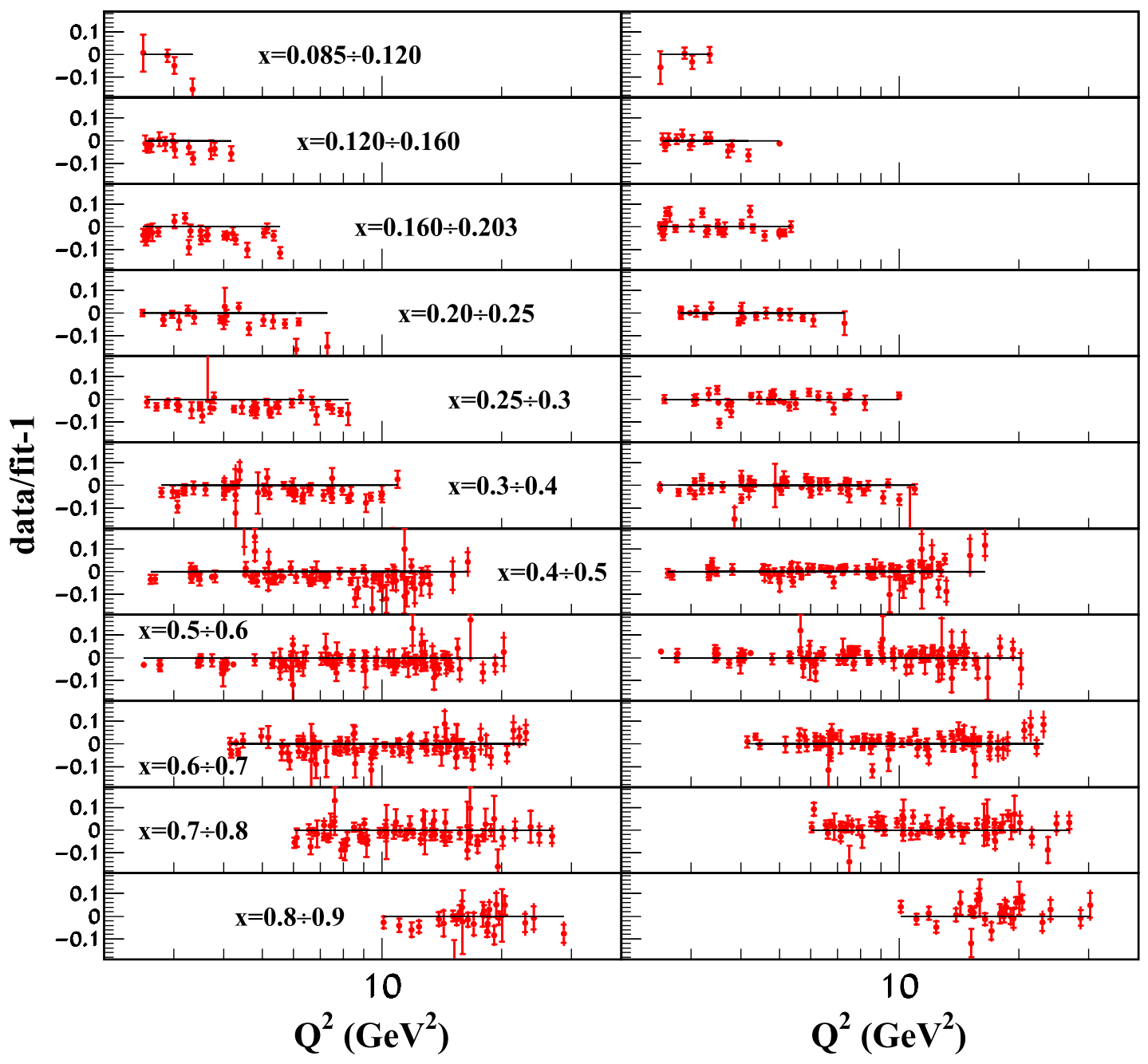

FIG. 7 (color online). Same as Fig. 2 for the pulls of the SLAC inclusive DIS cross section data of Refs. [77-81] for the proton target (left) and the deuterium target (right).

obtained for the individual data sets both stabilize. Moreover, the values of $\alpha_{s}\left(M_{Z}\right)$ obtained by individual experiments, capable to measure $\alpha_{s}$ from their data alone, have to be consistently reproduced. One observes a decreasing sequence of differences $\left|\Delta \alpha_{s}\left(M_{Z}\right)\right|$ between the sequential orders; see e.g., Refs. [60,103].

TABLE V. Parameters of the twist- 4 contribution to the DIS structure functions in Eq. (2.12) for the fit to NNLO accuracy in QCD.

\begin{tabular}{lccc}
\hline \hline & $H_{2}^{\mathrm{p}}(x) / \mathrm{GeV}^{2}$ & $H_{2}^{\mathrm{ns}}(x) / \mathrm{GeV}^{2}$ & $H_{T}^{\mathrm{p}}(x) / \mathrm{GeV}^{2}$ \\
\hline$x=0.1$ & $-0.036 \pm 0.012$ & $-0.034 \pm 0.023$ & $-0.091 \pm 0.017$ \\
$x=0.3$ & $-0.016 \pm 0.008$ & $0.006 \pm 0.017$ & $-0.061 \pm 0.012$ \\
$x=0.5$ & $0.026 \pm 0.007$ & $-0.0020 \pm 0.0094$ & $0.0276 \pm 0.0081$ \\
$x=0.7$ & $0.053 \pm 0.005$ & $-0.029 \pm 0.006$ & $0.031 \pm 0.006$ \\
$x=0.9$ & $0.0071 \pm 0.0026$ & $0.0009 \pm 0.0041$ & $0.0002 \pm 0.0015$ \\
\hline \hline
\end{tabular}

In the present analysis based on the measured scattering cross sections and with account of higher twist contributions, c.f. also Ref. [27], we obtain from the data sets described in Sec. III,

$$
\begin{gathered}
\alpha_{s}\left(M_{Z}\right)=0.1180 \pm 0.0012 \text { at NLO, } \\
\alpha_{s}\left(M_{Z}\right)=0.1134 \pm 0.0011 \text { at NNLO. }
\end{gathered}
$$

The value at NNLO is shifted by $\Delta \alpha_{s}\left(M_{Z}\right)=0.0046$ downward if compared to the NLO value. This range of uncertainty is well compatible with the scale uncertainty observed in a variation of the factorization and renormalization scales at NLO of $O(0.0050)$; cf. [104]. The present data allow a measurement of $\alpha_{s}\left(M_{Z}\right)$ with an accuracy of $O(1 \%)$. Therefore, NNLO analyses are mandatory, since the NLO results exhibit much too large theory errors. The response to the fitted $\alpha_{s}$ dependence is measured using the 


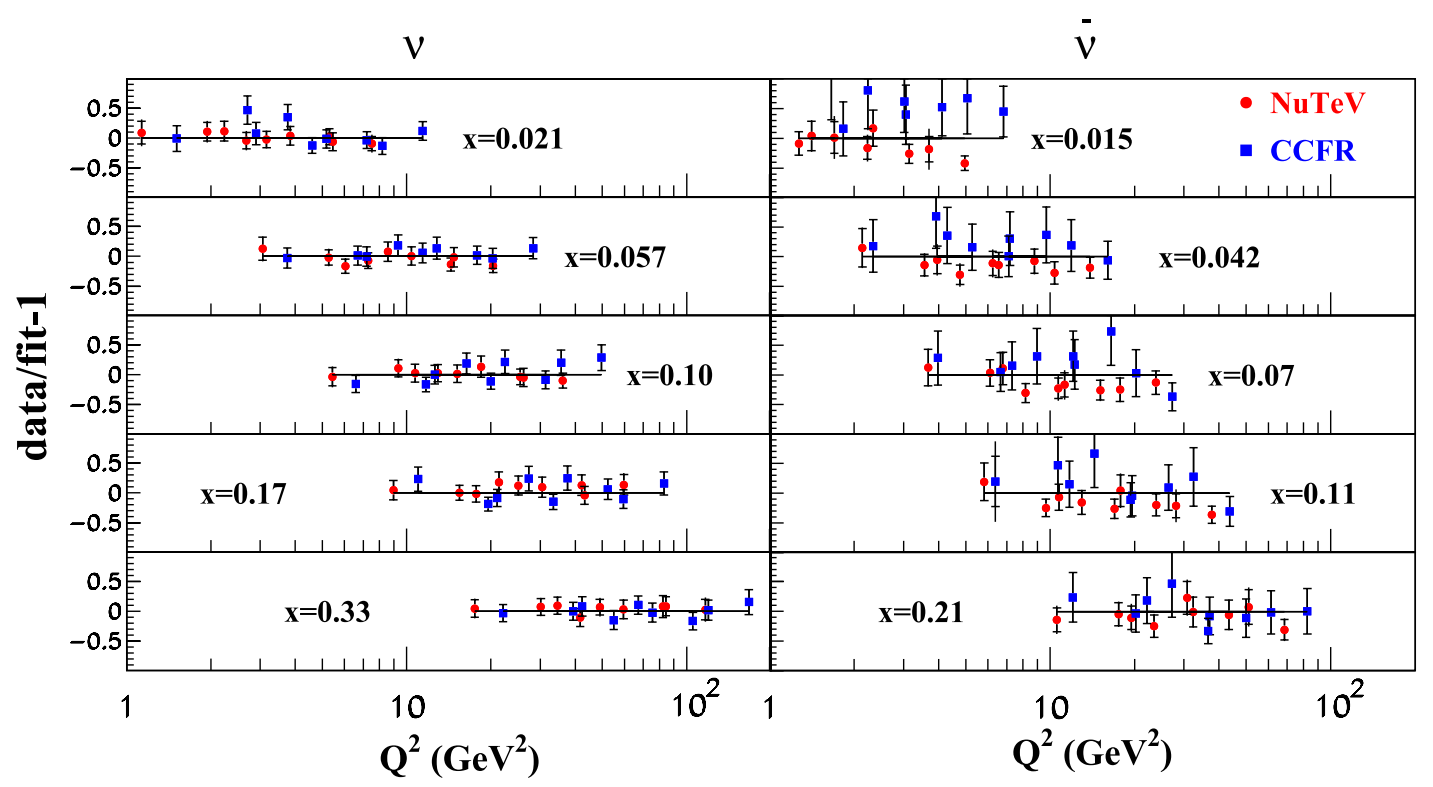

FIG. 8 (color online). Same as Fig. 2 for the pulls of neutrino (left) and antineutrino (right) induced dimuon production cross section data of Ref. [95] (circles: NuTeV experiment; squares: CCFR experiment).

$\chi^{2}$ functional of Eq. (A1). In Fig. 17 the dependence of $\chi^{2}$ of $\alpha_{s}\left(M_{Z}\right)$ is illustrated at NLO and NNLO.

In Table VI we compare the values for $\alpha_{s}\left(M_{Z}\right)$ obtained for the individual data sets at NLO and NNLO in the present fit and with results obtained by some of the experiments. Here the $\alpha_{s}$ value for BCDMS was reevaluated using the value of $\Lambda_{\mathrm{QCD}}^{\mathrm{NLO}}=224 \mathrm{MeV}$ and $\alpha_{s}(10 \mathrm{GeV})=$ 0.160 [83]. These values correspond to a NLO fit with $n_{f}=4$ in the $\overline{\mathrm{MS}}$ scheme. We evolved this value back to the charm threshold keeping $n_{f}=4$ and determined then $\alpha_{s}\left(M_{Z}\right)$ evolving forward passing the bottom threshold. In
Ref. [59] higher twist contributions and $\Lambda_{\mathrm{QCD}}^{n_{f}=4}$ were fitted together for the BCDMS $\mu p$ and $\mu d$ data resulting in the somewhat larger value $\Lambda_{\mathrm{QCD}}^{n_{f}=4}=263 \pm 42 \mathrm{MeV}$ and the NLO value $\alpha_{s}\left(M_{Z}\right)=0.113 \pm 0.003(\exp )$, which was also obtained $[60,103]$. Both values are compatible within errors.

In Fig. 18 we plot the $\chi^{2}$ profile using Eq. (A1) at NLO and NNLO. To that end, we compare the fit result with the $\alpha_{s}$ behavior of the individual data set, fixing all other parameters. The minimum and variation $\left(\Delta \chi^{2} \equiv 1\right)$ then
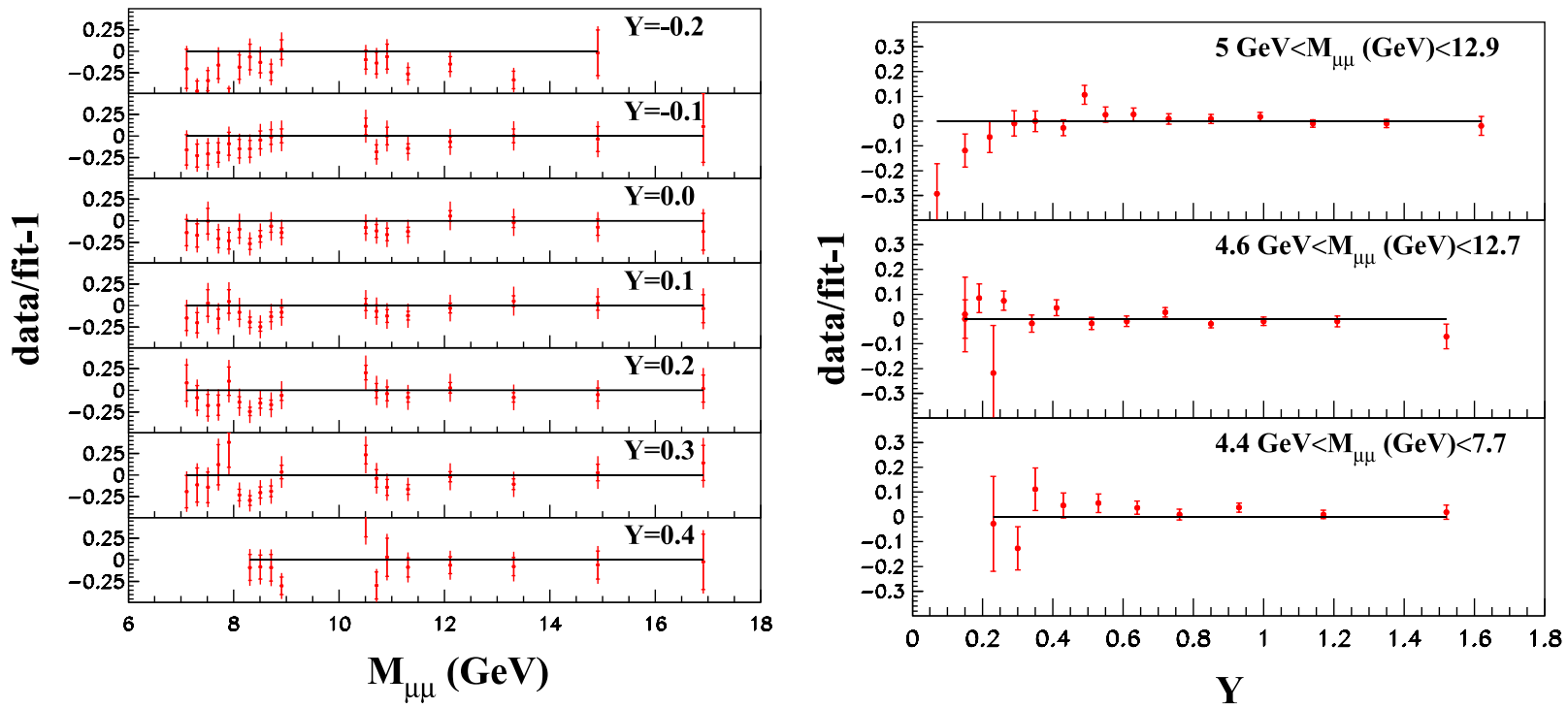

FIG. 9 (color online). Same as Fig. 2 for the pulls of the DY process cross section data of Ref. [90] binned in the muon pair rapidity $Y$ vs the invariant mass $M_{\mu \mu}$ of the muon pair (left) and the ones of Ref. [91] binned in $M_{\mu \mu}$ vs $Y$ (right). 

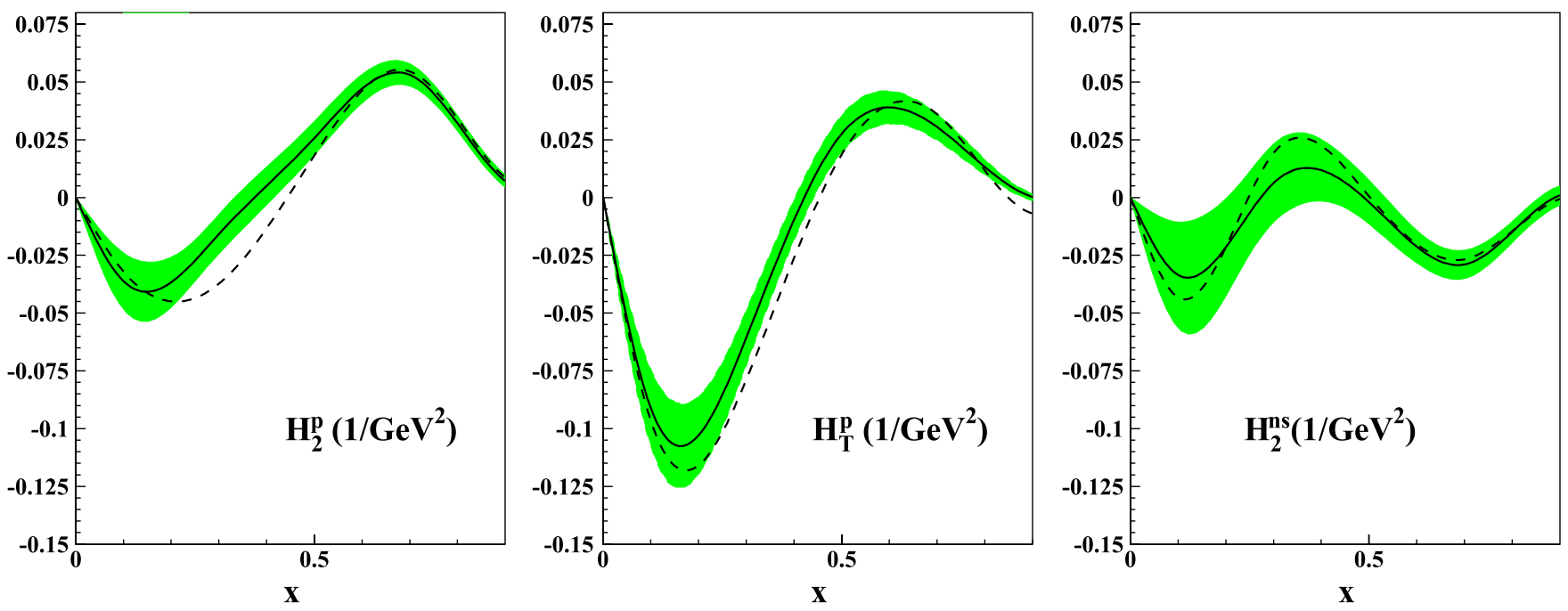

FIG. 10 (color online). Central values (solid line) and the $1 \sigma$ bands (shaded area) for the coefficients of the twist-4 terms of Eq. (2.12) in the inclusive DIS structure functions obtained from our NNLO fit (left: $F_{2}$ of the proton; middle: $F_{T}$ of the proton; right: nonsinglet $F_{2}$ ). The central values of the twist-4 coefficients obtained from our NLO fit are shown for comparison (dashed line).

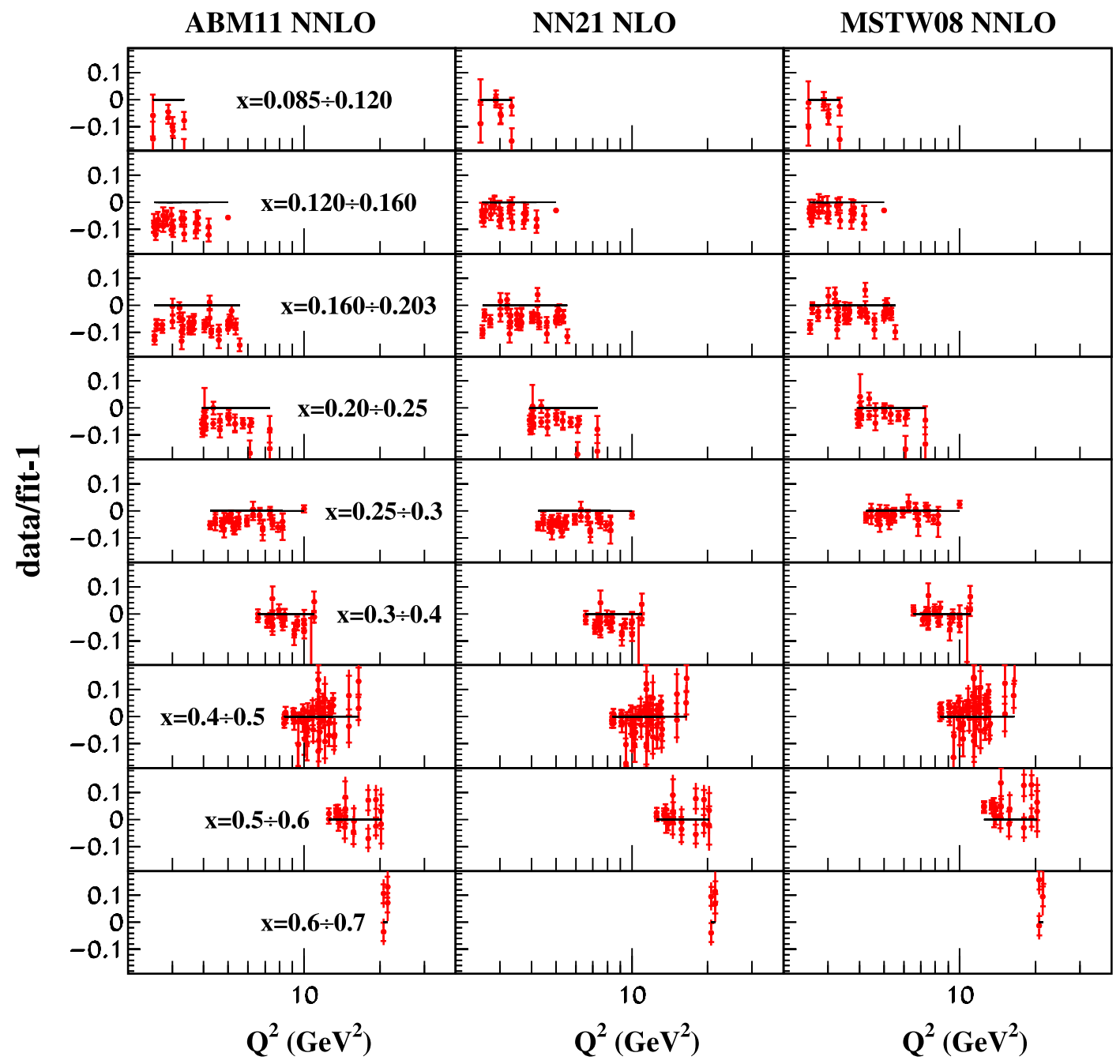

FIG. 11 (color online). Same as Fig. 7 without the HT terms taken into account and for various 3-flavor PDFs (left: present analysis; right: MSTW [22]). NLO calculations based on the 3-flavor NLO NN21 PDFs [100] are given for comparison (middle). Only the data surviving after the cut of Eq. (4.11) are shown; the proton and deuterium data points are superimposed. 


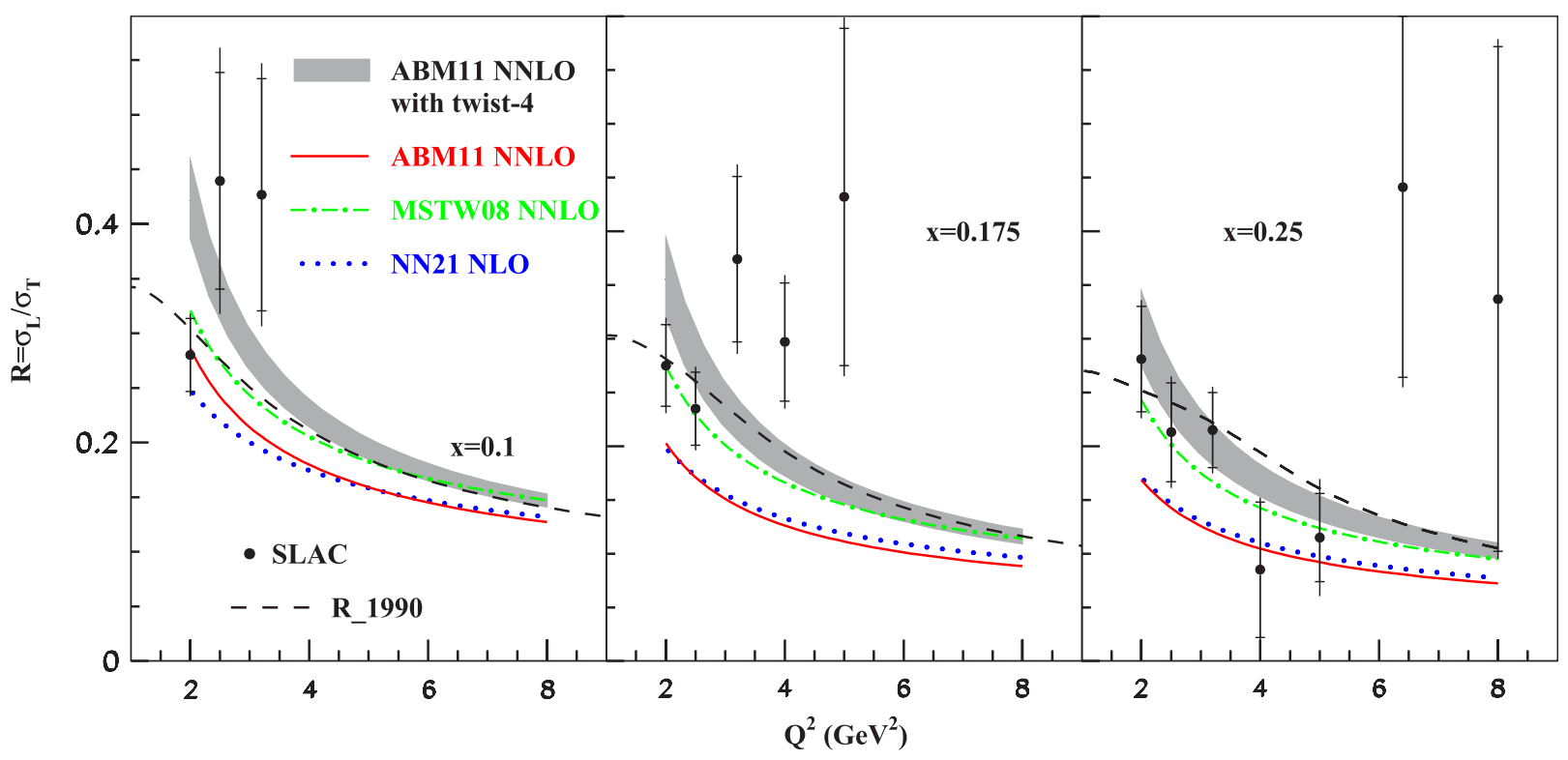

FIG. 12 (color online). Shaded area gives $1 \sigma$ band of the NNLO predictions for the ratio $R=\sigma_{L} / \sigma_{T}$; cf. Eq. (3.2), based on the ABM11 PDFs and the twist-4 terms obtained from our fit at different values of $x$ vs the momentum transfer $Q^{2}$. Central values of the NNLO predictions for $R$ based on the MSTW PDFs [22] (dashed-dotted line), NNLO predictions based on the ABM11 PDFs (solid line), and NLO predictions based on the NN21 PDFs [100] (dotted line), all taken in the 3-flavor scheme and without twist-4 terms, are given for comparison. Data points show values of $R$ extracted from the SLAC proton and deuterium data [62] with the empirical parameterization of those data $R_{1990}$ obtained in Ref. [62] superimposed (dashed line).

determine the values given in Table VI; see also Fig. 18. For BCDMS and NMC we find complete consistency to the values given by the experiments and the present analysis. The downward shift $\Delta \alpha_{s}\left(M_{Z}\right)$ that is consistently observed when going from NLO to NNLO amounts to values between 0.0030 and 0.0055 , with a lower sensitivity for the DY data, which yield rather low values with large errors. The fitted central values are well covered by the individual data sets. Figure 18 shows the response with respect to $\alpha_{s}\left(M_{Z}\right)$ of the individual data sets fixing the nonperturbative shape parameters in the global fit. One may refit these parameters in changing $\alpha_{s}\left(M_{Z}\right)$; cf. Fig. 19. However, here the change in the other PDF parameters remains undocumented, in particular, if the corresponding covariance matrices are not publicly available, as is the case for some of the global fits. We have performed this analysis only to compare to the MSTW and NNPDF analyses below but still prefer the results of Fig. 18. Both results are given in Table XI for comparison. In comparing Figs. 18 and 19, one finds that the shape parameters in the case of the BCDMS and HERA data remain widely stable, and larger shifts are introduced for the NMC and SLAC data. The stability of the results, on the other hand, allows one to conclude that fully compatible sets of precision data were used.

We have also performed NLO fits, including the Tevatron jet data [108-111]. Furthermore, we have formally extended the analysis fitting the DIS and DY data at NNLO while treating the Tevatron jet data at NLO and supplementing threshold corrections based on soft gluon resummation [112] for the single jet inclusive data. This latter approximation we denoted by NNLO*; cf. Ref. [102]. A NLO measurement of $\alpha_{s}\left(M_{Z}\right)$ was also performed by CDF [113], with larger errors than in Ref. [114], $\alpha_{s}\left(M_{Z}\right)=0.1178_{-0.0095}^{+0.0081}(\mathrm{exp})_{-0.0047}^{+0.007}(\mathrm{scale}) \pm 0.0059(\mathrm{PDF})$. At NLO the different sets of Tevatron jet data do not modify the value obtained in our standard analysis. A consistent NNLO is not yet possible since the corresponding scattering cross sections still have to be calculated. Again a systematic downward shift of $\Delta \alpha_{s}\left(M_{Z}\right)=$ 0.0029-0.0047 is obtained upon going from NLO to NNLO*. The corresponding central values are $1 \sigma$ compatible with our NNLO central value in Eq. (4.13). We would like to mention that already our former ABKM09 results [16] give a very good description of the CMS jet data [115] and also the Tevatron 3-jet data [116]. We note that in a recent NLO analysis of the 5-jet cross section at LEP a value of $\alpha_{s}\left(M_{Z}\right)=0.1156_{-0.0034}^{+0.0041}$ was obtained [117].

The higher twist terms play an important role in the determination of $\alpha_{s}$ from the DIS data [59]. In our analysis they contribute up to $10 \%$ of the cross section at the low margin of $Q^{2}$ and $W$ given by Eq. (3.3). As a result, the value of $\alpha_{s}$ is strongly correlated with the twist-4 coefficients, which are extracted from the fit simultaneously with $\alpha_{s}$; cf. Fig. 20. The correlation is more pronounced for $\mathrm{H}_{2}$ at large $x$ and for $H_{T}$ at small $x$. The latter affects the determination of $\alpha_{s}$ even in the case of a more stringent cut on $W$ since the low- $x$ part of the data is not sensitive to this 


\section{$\mu=2 \mathbf{G e V}, \mathbf{n}_{\mathbf{f}}=\mathbf{4}$}
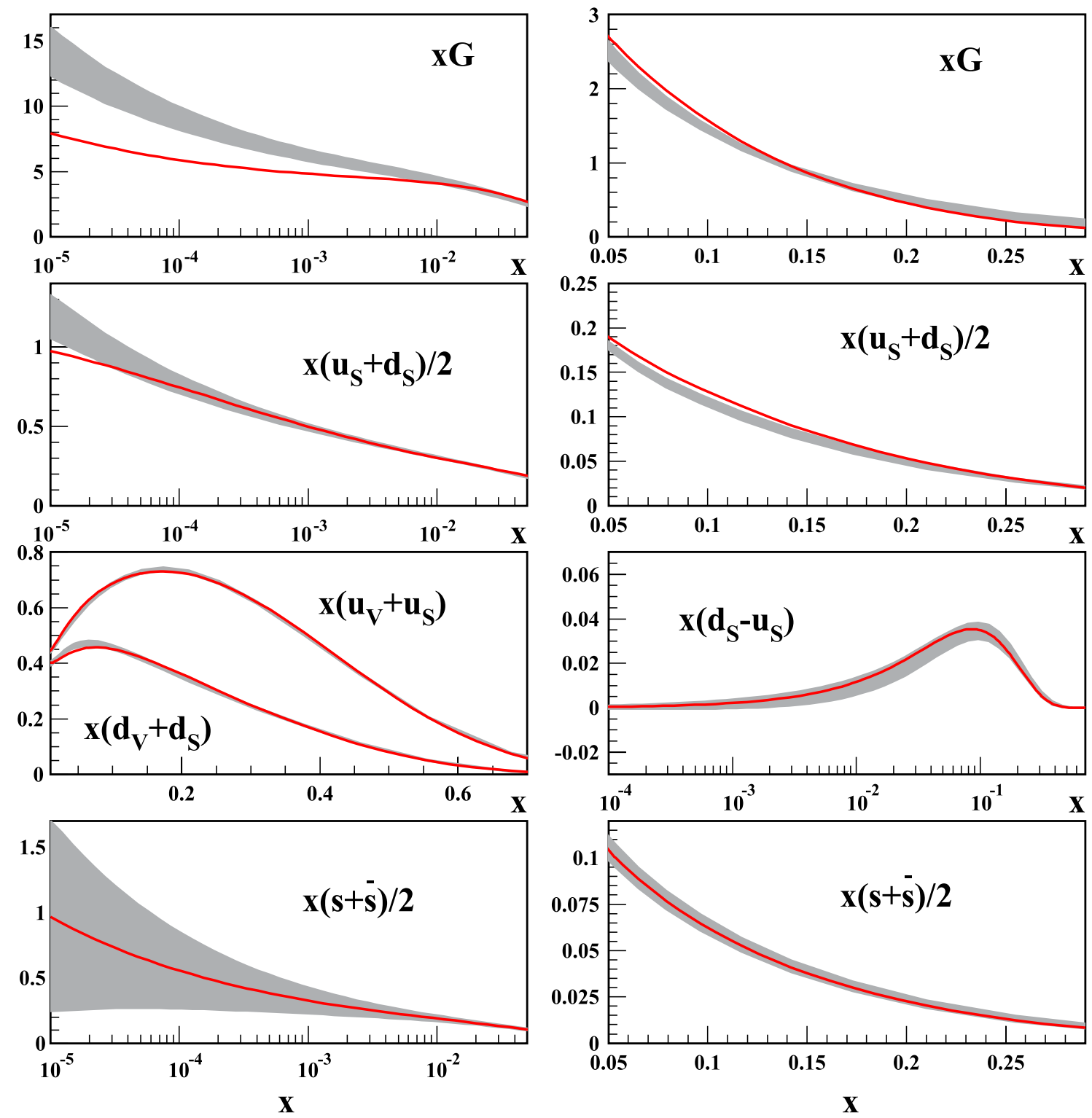

FIG. 13 (color online). $1 \sigma$ band for the 4-flavor NNLO ABKM09 PDFs [16] at the scale of $\mu=2$ GeV vs $x$ (shaded area) compared with the central values for ones of this analysis (solid lines).

cut. For the variant of our NNLO fit with the cut of Eq. (4.11) imposed and the higher twist terms set to zero, we obtain the value of $\alpha_{s}\left(M_{Z}\right)=0.1191(6)$, much bigger than our nominal result of Eq. (4.13). For comparison, the same fit with the higher twist terms fixed at the values of Table $\mathrm{V}$ gives $\alpha_{s}\left(M_{Z}\right)=0.1131(5)$ comparable with Eq. (4.13). Note that in both cases the error in $\alpha_{s}$ is smaller than the one of Eq. (4.13) despite the reduced data set used in the fit. This says, that the uncertainty in $\alpha_{s}$ is essentially controlled by the higher twist term variation. To get rid of the impact of the higher twist terms on $\alpha_{s}$, an even more stringent cut on $Q^{2}$ is necessary, in addition to the cut on $W$. With the NNLO variant of our fit and using

$$
W^{2}>12.5 \mathrm{GeV}^{2}, \quad Q^{2}>10 \mathrm{GeV}^{2},
$$

the value of $\alpha_{s}\left(M_{Z}\right)=0.1134(8)$ is obtained, if the higher twist terms are set to zero and $\alpha_{s}\left(M_{Z}\right)=0.1135(8)$, if the higher twist terms are fixed at the values of Table V. From this comparison we conclude that $\alpha_{s}$ is pushed to larger values due to the neglect of the higher twist terms in the case of a cut as in Eq. (4.11), which is commonly imposed in the global PDF fits. Likewise, it is less sensitive to the details of the fit ansatz. As we found earlier [27], the value of $\alpha_{s}$ extracted from our fit is quite sensitive to the treatment of the NMC data [88]. Normally, we use the NMC data on the cross section in the fit; cf. Sec. III A. If, 


\section{$\mu=2 \mathrm{GeV}, \mathbf{n}_{\mathbf{f}}=4$}
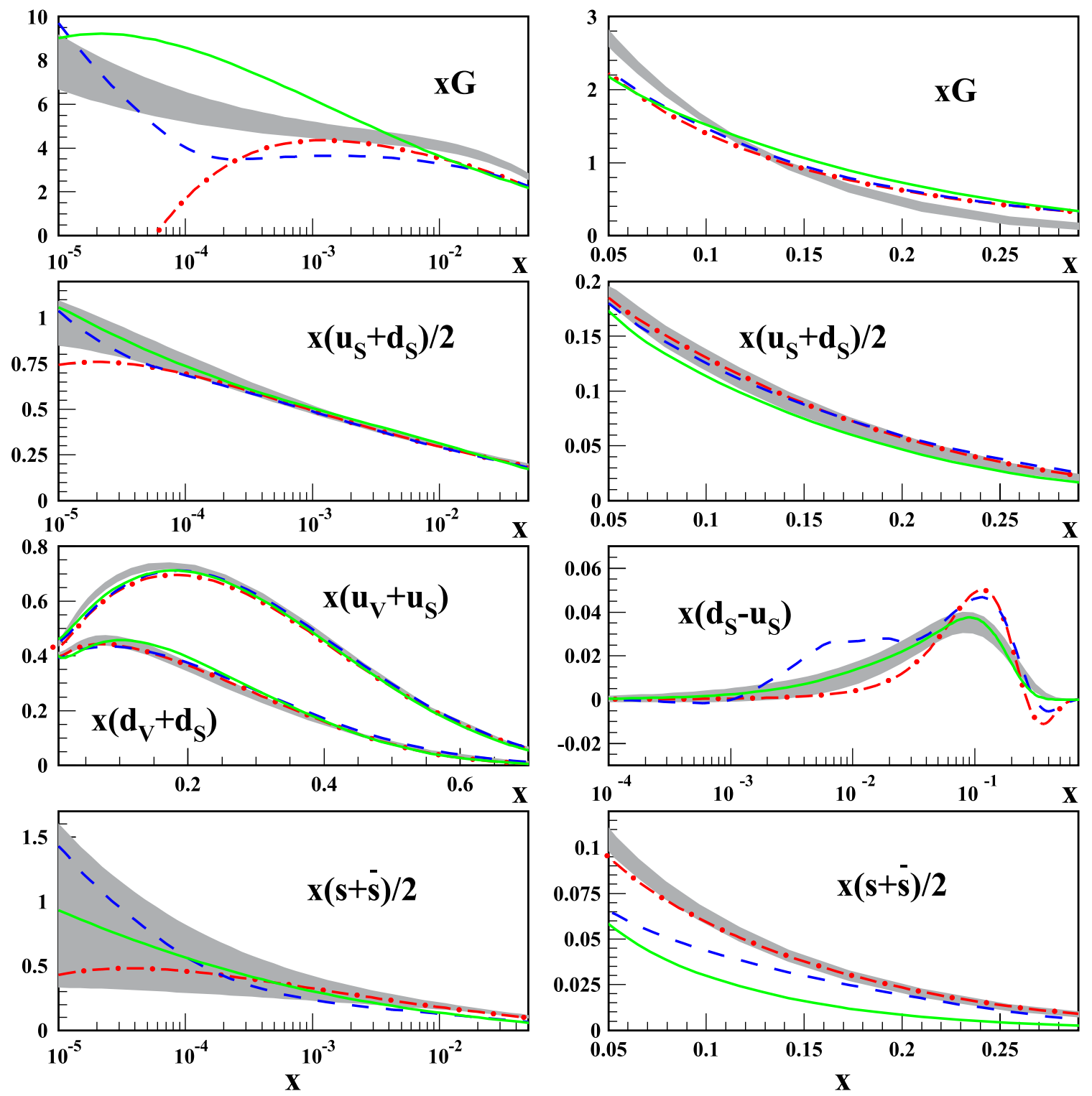

FIG. 14 (color online). $1 \sigma$ band for the 4-flavor NNLO ABM11 PDFs at the scale of $\mu=2$ GeV vs $x$ obtained in this analysis (shaded area) compared with the ones obtained by other groups (solid lines: JR09 [20]; dashed-dotted lines: MSTW [22]; dashed lines: NN21 [23]).

however, we employ instead the NMC data on $F_{2}$ extracted by the NMC Collaboration with their own assumptions about the value of $R$, the value of $\alpha_{s}\left(M_{Z}\right)$ increases by +0.0035 for the case of our earlier ABKM09 NNLO fit [27]. In comparison, for the variant of the ABKM09 with the cut of Eq. (4.11) imposed and the high-twist terms set to zero, the value of $\alpha_{s}\left(M_{Z}\right)$ is shifted by +0.0003 only. This is in agreement with the size of the $\alpha_{s}$ variation obtained in the variant of the MSTW08 fit with an improved treatment of the NMC data [28]. Note, however, that the details of the DIS data treatment employed in the improved analysis of Ref. [28], are still different from ours. In places, this has an impact on the $\alpha_{s}$ value. For example, if we combine the errors in the NMC and HERA data in quadrature, as it is done in Refs. [22,28], the NNLO ABKM09 value of $\alpha_{s}\left(M_{Z}\right)$ is shifted upwards by +0.0029 and its sensitivity to the NMC data treatment is reduced to +0.0011 . This effect may, in particular, explain the relatively big value of $\alpha_{s}\left(M_{Z}\right)=0.1164$ observed in Ref. [28] with the cuts similar to ones of Eq. (4.14).

The impact of the NMC data treatment on the fit has also been studied for NN21 in Ref. [30], where little effect on the PDFs has been found. However, the value of $R$ obtained in that analysis is still much lower than the one obtained in 


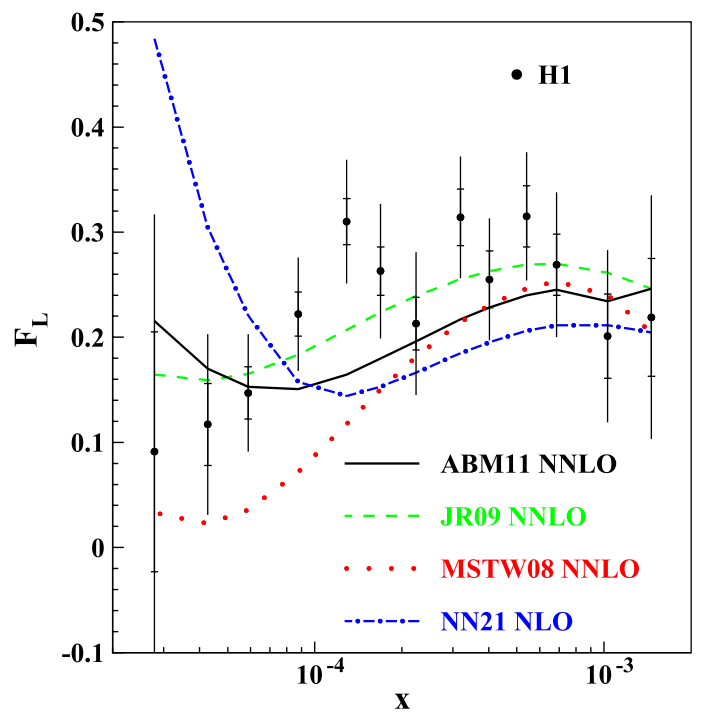

FIG. 15 (color online). Data on $F_{L}$ vs $x$ obtained by the H1 Collaboration [31] confronted with the 3-flavor scheme NNLO predictions based on the different PDFs (solid line: this analysis; dashed line: JR09 [20]; dotted line: MSTW [22]). NLO predictions based on the 3-flavor NN21 PDFs [100] are given for comparison (dashed-dotted line). Value of $Q^{2}$ for the data points and the curves in the plot rises with $x$ in the range of $1.5-45 \mathrm{GeV}^{2}$.

our fit and closer to the value of $R$ used by NMC to extract the value of $F_{2}$ from the cross section (compare Fig. 2 in Ref. [30] and Fig. 1 in Ref. [27]). Note in this context that the value of $\chi^{2} / N D P \approx 1.7$ obtained in Ref. [30] for the NMC data is much bigger than in our case; cf. Table IV. Furthermore, in the NN21 study the value of $\alpha_{s}$ is fixed. Therefore, its correlation with the PDFs is not considered. Finally, the expressions for cross sections used in the NN21 analysis do not include the power corrections in $Q^{2}$, which are numerically important at small $Q^{2}$ (compare Eqs. (2), (3) in Ref. [30] with Eq. (1) in Ref. [88], and Eqs. (3.1) and (3.2) of the present article). These differences make a detailed comparison of the results of Ref. [27,30] difficult.

The value of $\alpha_{s}$ obtained in our fit is substantially constrained by the BCDMS data of Refs. [82,83], which almost entirely survive after the cut of Eq. (3.3). Meanwhile, the authors of Ref. [118] suggested to cut in addition the most inaccurate BCDMS data with low inelasticity $y$. The value of $\alpha_{s}\left(M_{Z}\right)$ reported in Ref. [118] with such cut is by $\sim 0.009$ larger than the one obtained from the analysis of the whole set of the BCDMS data at NNLO. The pulls of the low- $y$ data rejected in the analysis of Ref. [118] with respect to our NNLO fit are given in Fig. 21. The fit is in reasonable agreement with data within the errors. Furthermore, rejecting these data points from the NNLO fit, we obtain a value of $\alpha_{s}\left(M_{Z}\right)=0.1139(12)$, which is somewhat bigger than the one in Eq. (4.13). The statistical significance of the shift, however, is marginal. The discrepant findings of Ref. [118] concerning the impact of the low- $y$ BCDMS data may appear due to the fact that the systematic uncertainties in the data are not taken into account in Ref. [118]. In our case the systematic errors are included into the value of $\chi^{2} ; \mathrm{cf}$. Sec. A. Therefore, the low- $y$ data points with an enhanced systematic uncertainty have reduced weight and do not affect the fit.

We turn now to comparisons with other NNLO analyses that will be performed studying the contribution of different data sets to $\alpha_{s}\left(M_{Z}\right)$. We first compare to the flavor nonsinglet analyses [60,103]. In Ref. [103] the valence analysis is performed by accounting for the remnant sea quark and gluon contributions to $F_{2}\left(x, Q^{2}\right)$ in the region $x>0.35$ through the PDFs taken from Ref. [16]. The results are summarized in Table VIII. The values of $\alpha_{s}\left(M_{Z}\right)$ at NLO turn out to be lower than those obtained in singlet analyses; cf. Tables VI and XII. However, they are consistent within the scale variation errors. At NNLO both analyses lead to the same values. Also note the anticorrelation of the size of higher twist contributions in the large- $x$ region with the inclusion of higher orders at leading twist; cf. Refs. $[60,61,67,103]$. The next order, denoted by $\mathrm{N}^{3} \mathrm{LO}^{*}$, yields information on the remaining theoretical uncertainty. At $\mathrm{N}^{3} \mathrm{LO}^{*}$, the nonsinglet three-loop Wilson coefficients are used $[9,10]$ and the four-loop nonsinglet anomalous dimension is estimated with a Padé approximation and accounting for a $100 \%$ error. In fact the latter extrapolation agrees within $20 \%$ with the second moment of the nonsinglet four-loop anomalous dimension $[135,136]$. For the three experiments, which give the bulk information on $\alpha_{s}\left(M_{Z}\right)$, the shift due to the $\mathrm{N}^{3} \mathrm{LO}^{*}$ contributions amount to $\left|\Delta \alpha_{s}\left(M_{Z}\right)\right|=0.0002-0.0006$ and globally to 0.0007 . At NNLO, the $\alpha_{s}\left(M_{Z}\right)$ values of the individual data sets vary by 0.0032 , consistent within the $1 \sigma$ errors.

Next, in Table IX, we compare with the fit results of NN21 [106,137] for individual data sets for DIS and other hadronic hard scattering data. The labels for those data sets in Table IX follow the original notation of NN21 in Refs. [106,137] (and, likewise in Table X for MSTW [107]). The references corresponding to the data sets are given additionally. At NLO the $\alpha_{s}\left(M_{Z}\right)$ values range from 0.1135 (E866, DY) to 0.1252 (NuTeV), with a corresponding range at NNLO of 0.1111 (D0 jet) to 0.1225 (CDF jet). The value of $\alpha_{s}\left(M_{Z}\right)=0.1204 \pm 0.0015$ for the BCDMS data at NLO differs significantly from that given by the experiment $0.1111 \pm 0.0018$ [83]. Comparing the change of the $\alpha_{s}\left(M_{Z}\right)$ values between the NLO and NNLO analyses, one finds downward shifts between $0.0075(\mathrm{NuTeV})$ and 0.003 (CDF R2KT) and upward shifts between 0.0055 (CDF Zrap) and 0.0061 (ZEUS H2); see Table IX. The values for $\alpha_{s}\left(M_{Z}\right)$ obtained for the SLAC data are found to be larger than 0.124 both at NLO and NNLO; cf. Fig. 2 in Ref. [106]. The $\chi^{2}$ values for the scans in $\alpha_{s}\left(M_{Z}\right)$ at NNLO turn out to be worse than in NLO in the global analysis for the data sets of NMC, BCDMS, HERA I, CHORUS, ZEUS 


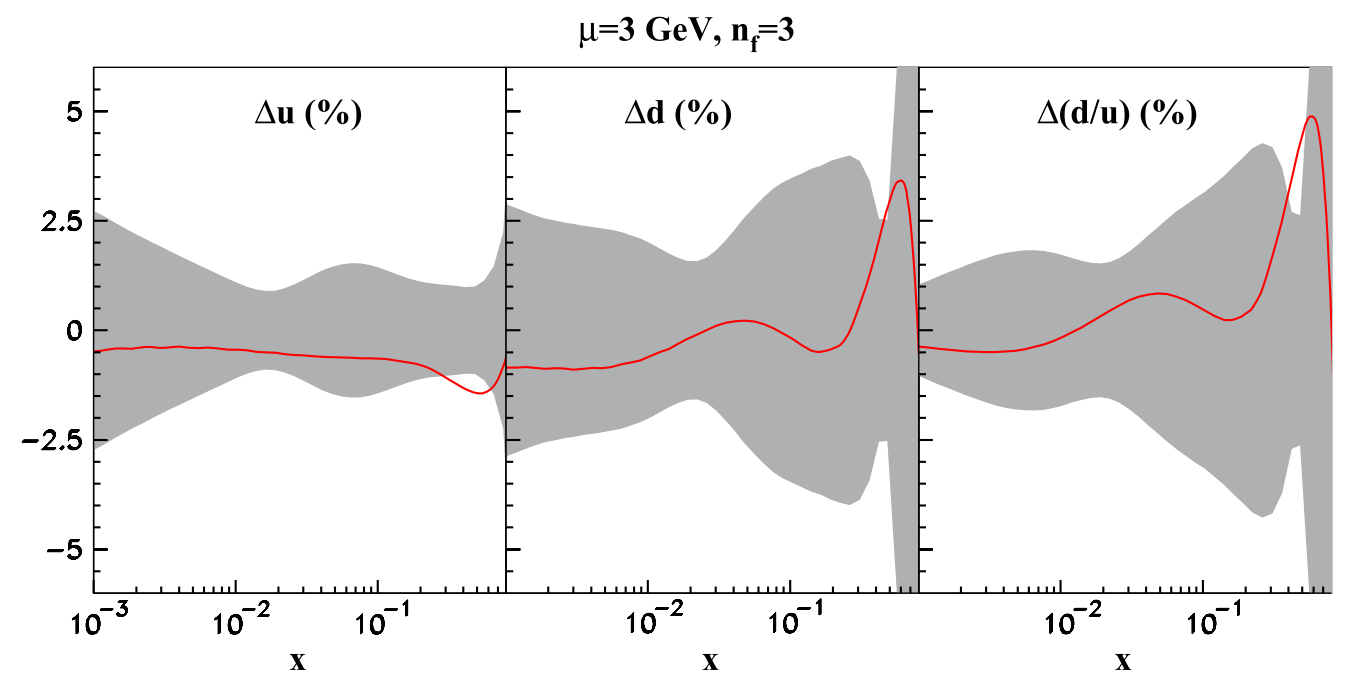

FIG. 16 (color online). $1 \sigma$ band for the 3-flavor NNLO $u$-quark distribution (left), $d$-quark distribution (middle), and the $d / u$ ratio (right) at the scale of $\mu=2 \mathrm{GeV}$ vs $x$ in comparison to the central value of the fit variant with the data normalization changed from the settings of Sec. III to the ones of Ref. [22] (solid curves).

F2C, DY E866, CDF Zrap, and D0 Zrap; again see Fig. 2 in Ref. [106]. Comparing the DIS only fit to the global analysis, it is found that the $\chi^{2}$ values improve significantly, except for NMCp, and to a lesser extent for SLAC at higher values of $\alpha_{s}\left(M_{Z}\right)$; cf. Fig. 5 in Ref. [106].

In Table X the $\alpha_{s}$ values determined by MSTW at NLO and NNLO [107] are compared for individual data sets in the fit. Here, the $1 \sigma$ errors, as defined by MSTW, are read off the corresponding plots in Ref. [153]. This definition of

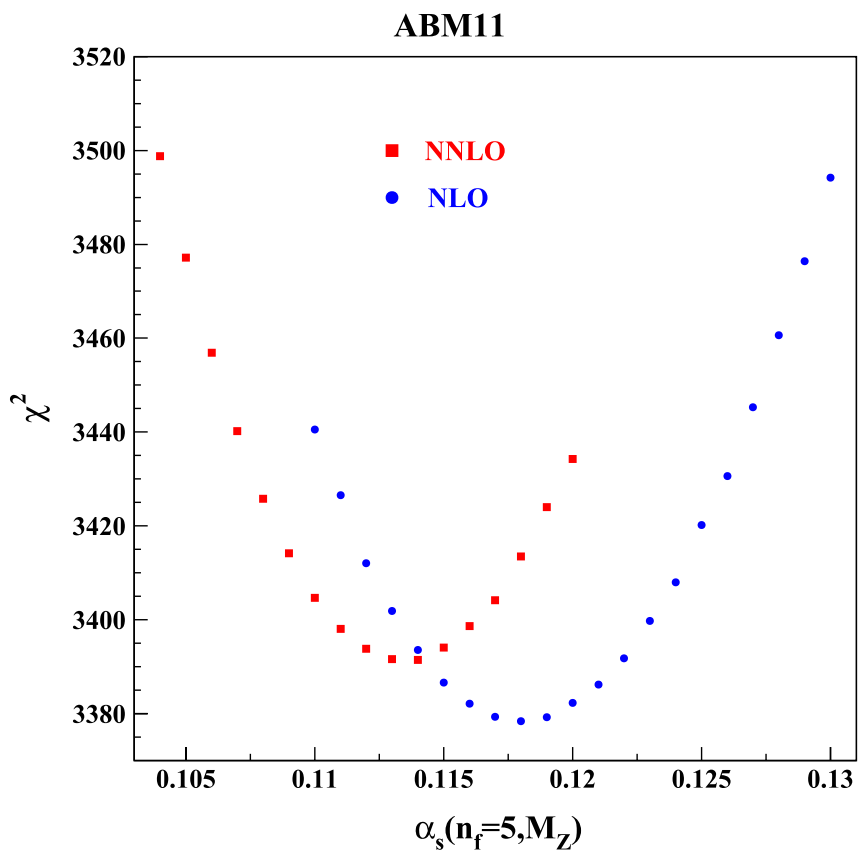

FIG. 17 (color online). $\quad \chi^{2}$-profile, Eq. (A1), as a function of $\alpha_{s}\left(M_{Z}\right)$ in the present analysis at NLO (circles) and NNLO (squares).
" $1 \sigma$ " is obtained for values of $\Delta \chi^{2}$ much larger than one. Moreover, these values do strongly vary between the different measurements used, which is unlike the case for the $\mathrm{ABM}$ and NN21 analyses. This procedure leads to an enlargement of errors, which, e.g., in the case of BCDMS translates into a NNLO value $\alpha_{s}\left(M_{Z}\right)=0.1085 \pm 0.0095$ rather than the experimental one of $\Delta \alpha_{s}\left(M_{Z}\right)= \pm 0.0018$. The latter accuracy is reflected in other analyses, however; cf. $\Delta \alpha_{s}\left(M_{Z}\right)= \pm 0.0007 \quad(\mathrm{BB}), \quad \Delta \alpha_{s}\left(M_{Z}\right)= \pm 0.0015$ (NN21), and $\Delta \alpha_{s}\left(M_{Z}\right)= \pm 0.0013$ (ABM11). Similar effects are present for various other data sets, as can be seen by comparing the values given in Tables VI, VII, VIII, IX, and $\mathrm{X}$. In this way, almost all individual $\alpha_{s}\left(M_{Z}\right)$ errors, even at NNLO, are larger than the typical theory uncertainty of about \pm 0.0050 at NLO. We stress that the present analysis (ABM11) correctly accounts for the experimental systematic errors of all data sets used; cf. Tables VI and VII, and that an enlargement of errors has not been necessary. In view of this fact, it is somewhat surprising that the final error in the MSTW analysis, i.e., $\Delta \alpha_{s}\left(M_{Z}\right)=$ \pm 0.0014 (MSTW), fully agrees with those obtained by BB, ABM11, and NN21; cf. Table XI. Unlike the case of NN21, the $\alpha_{s}\left(M_{Z}\right)$ values of MSTW become generally lower at NNLO if compared to NLO, with the exception of the D0 run II $Z$-boson rapidity data set, where the NNLO value is slightly higher.

It is also interesting to compare the $\alpha_{s}\left(M_{Z}\right)$ values obtained in the NN21 [106,137], MSTW [107], and ABM11 analyses with respect to the individual data sets used in those fits. At NLO, NN21 obtains lower values for D0 R2CON than MSTW and ABM and a significantly higher value for D0 ZRAP than MSTW; see Tables IX and X. At NNLO, the individual $\alpha_{s}\left(M_{Z}\right)$ value for the data set CDF Zrap moves upward with respect to the NLO value with a significantly larger error, while for MSTW the value 
TABLE VI. Comparison of the values of $\alpha_{s}\left(M_{Z}\right)$ obtained by BCDMS [83] and NMC [105] at NLO with the individual results of the fit in the present analysis at NLO and NNLO for the HERA data [18,31], the NMC data [84], the BCDMS data [82,83], the SLAC data [62,77-81], and the DY data $[90,91]$.

\begin{tabular}{lccc}
\hline \hline & & $\alpha_{s}\left(M_{Z}\right)$ & \\
Experiment & $\mathrm{NLO}_{\exp }$ & $\mathrm{NLO}$ & $\mathrm{NNLO}$ \\
\hline BCDMS & $0.1111 \pm 0.0018$ & $0.1150 \pm 0.0012$ & $0.1084 \pm 0.0013$ \\
NMC & $0.117_{-0.016}^{+0.011}$ & $0.1182 \pm 0.0007$ & $0.1152 \pm 0.0007$ \\
SLAC & & $0.1173 \pm 0.0003$ & $0.1128 \pm 0.0003$ \\
HERA comb. & $0.1174 \pm 0.0003$ & $0.1126 \pm 0.0002$ \\
DY & & $0.108 \pm 0.010$ & $0.101 \pm 0.025$ \\
ABM11 & $0.1180 \pm 0.0012$ & $0.1134 \pm 0.0011$ \\
\hline \hline
\end{tabular}

remains the same as at NLO. The values of ABM11 given in Table VII for Tevatron jet data are rather close to those of MSTW, both at NLO an NNLO*. For the NuTeV data the NLO and NNLO $\alpha_{s}\left(M_{Z}\right)$ values show a bigger difference for NN21 than for MSTW, while the NNLO values are rather similar.

In Table XI we compare the $\alpha_{s}$ values of the ABM11, BBG, NN21, and MSTW analyses for those data sets that are commonly used at NNLO. An NLO comparison would still be subject to a scale error of $\sim 0.0050$, which is usually too large to differentiate between the various fit results. For ABM11 we present the $\alpha_{s}$ values extracted from Figs. 18 and 19. For the BCDMS data ABM11, BBG, and MSTW obtain lower values, while NN21 differs, e.g., by $+2 \sigma$ [or $\Delta \alpha_{s}\left(M_{Z}\right)=0.0030$ ] from MSTW and $+7 \sigma$ [or $\left.\Delta \alpha_{s}\left(M_{Z}\right)=0.0110\right]$ from ABM11. For the NMC data $\mathrm{ABM}, \mathrm{BBG}$, and NN21 do agree very well, while the value of MSTW shows an upward shift of $\Delta \alpha_{s}\left(M_{Z}\right)=0.0066$

ABM11
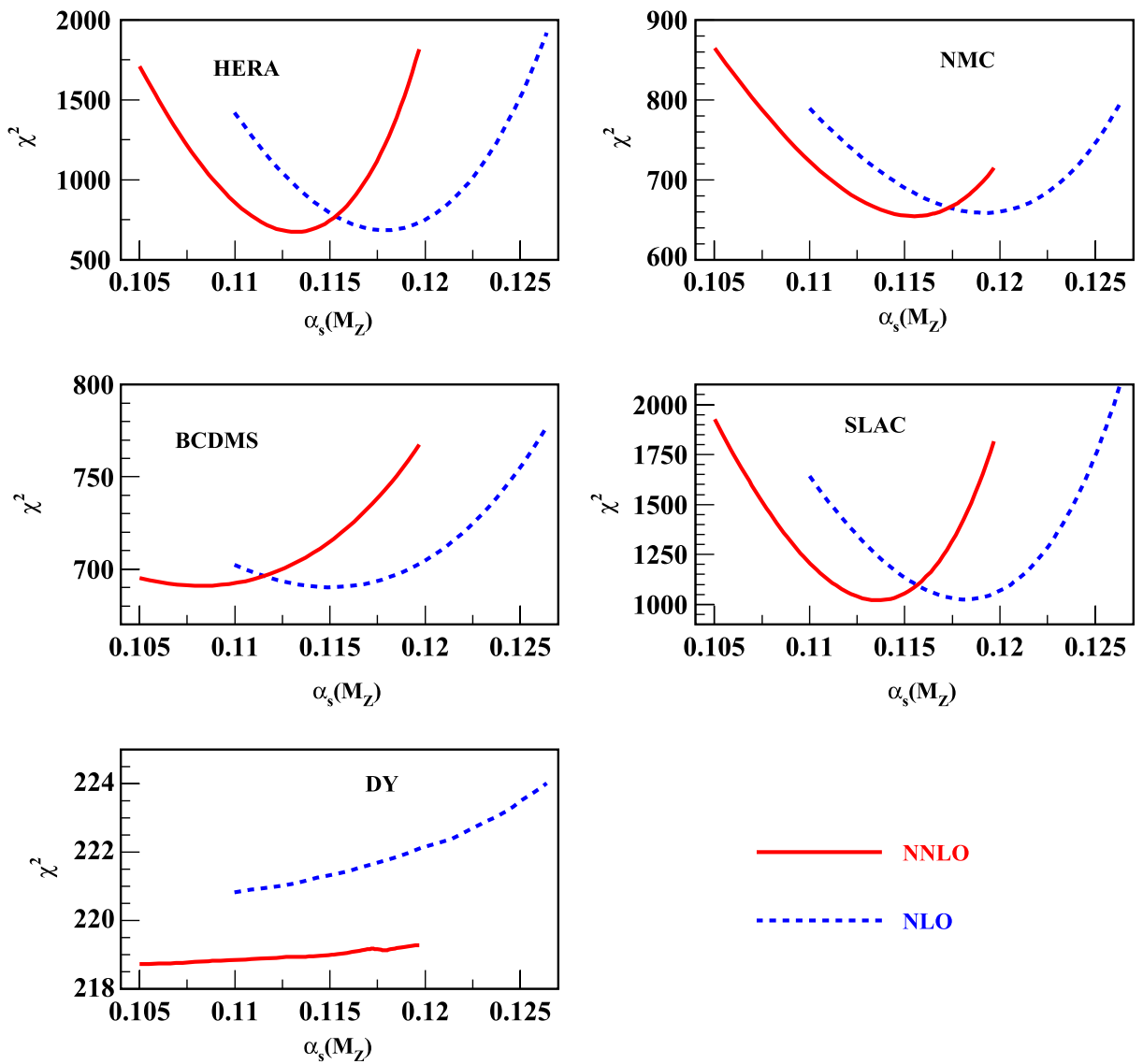

FIG. 18 (color online). $\quad \chi^{2}$-profile vs the value of $\alpha_{s}\left(M_{Z}\right)$, Eq. (A1), for the data sets of Table VI, all calculated with the PDF and HT parameters fixed at the values obtained from the fits with $\alpha_{s}\left(M_{Z}\right)$ released (solid lines: NNLO fit; dashed lines: NLO fit). 
ABM11
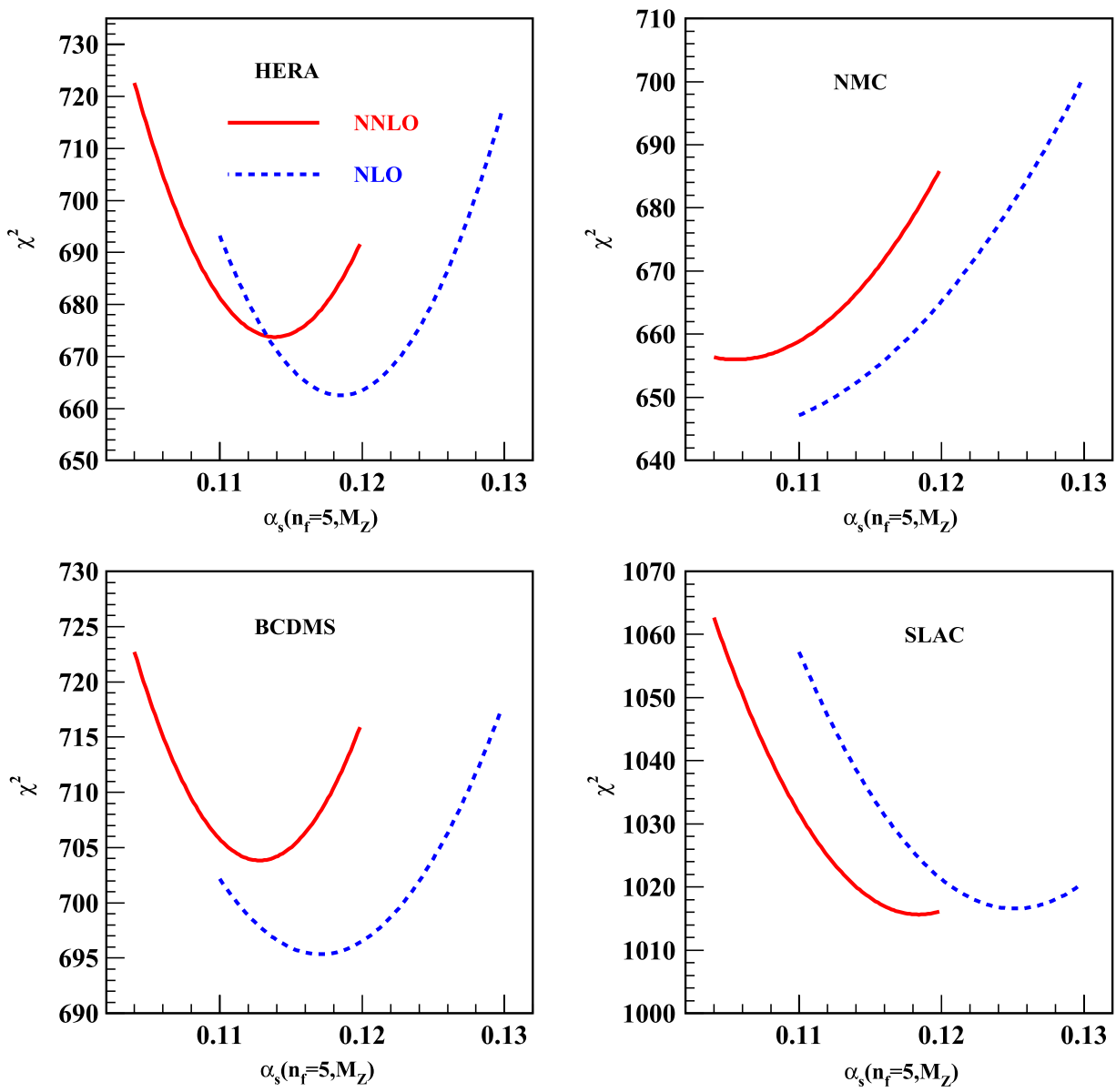

FIG. 19 (color online). $\quad \chi^{2}$-profile vs the value of $\alpha_{s}\left(M_{Z}\right)$, Eq. (A1), for the data sets of Table VI all obtained in variants of the present analysis with the value of $\alpha_{s}$ fixed and all other parameters fitted (solid lines: NNLO fit; dashed lines: NLO fit).

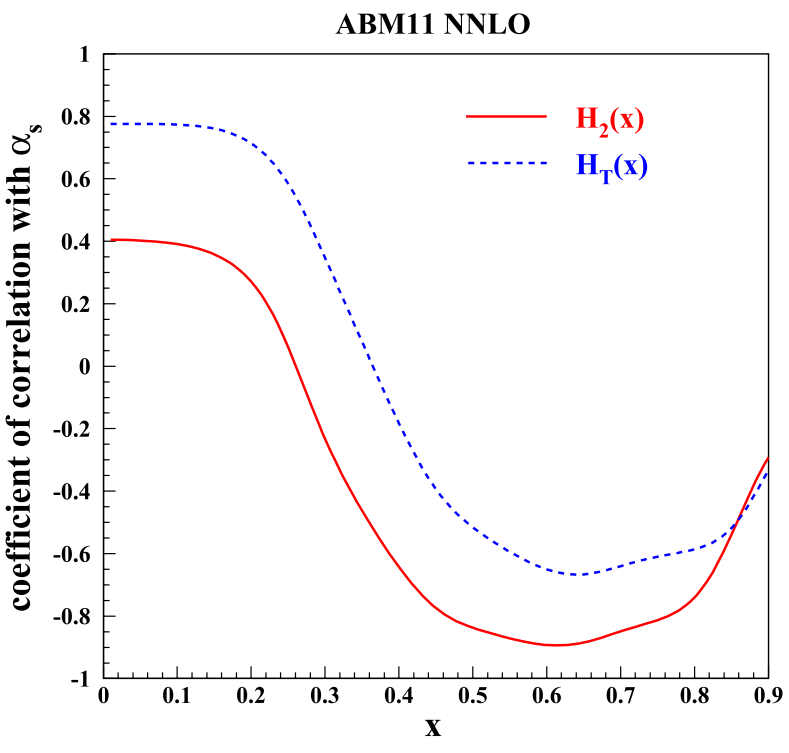

FIG. 20 (color online). Correlation coefficient of $\alpha_{s}\left(M_{Z}\right)$ with the nucleon twist-4 coefficients $H_{2}$ (solid line) and $H_{T}$ (dashed line) vs $x$ as obtained in our NNLO fit. compared to NN21. The size of both of these shifts is of the order of NLO scale uncertainty and should not be present at NNLO. For the SLAC ep data, the values by ABM11, BBG, and MSTW are consistent within errors. On the other hand, MSTW reports a much larger value for the SLAC ed data than obtained by ABM11 and BBG. NN21 obtains partial $\alpha_{s}\left(M_{Z}\right)$ values $>0.124$ both at NLO and NNLO both in their global and DIS only analyses. This is in contrast to the present results, to $\mathrm{BB}$, and to MSTW for the $e p$ data. In the nonsinglet $\mathrm{BBG}$ analysis the influence of the HERA data is strongly reduced, since most of these data are located within the quark-sea region. The fit results of NN21 and MSTW lead to values of $\alpha_{s}\left(M_{Z}\right) \sim 0.120$ while those of ABM11 yield a much lower value of 0.1139 (0.1126). Note that the MSTW analysis does not yet include the HERA run I combined data set [18]. The results for the DY data are consistent between ABM11 and MSTW, although the sensitivity of these data to $\alpha_{s}\left(M_{Z}\right)$ is comparably small. In summary, despite the fact that NN21 and MSTW obtain nearly the same global fit values for $\alpha_{s}\left(M_{Z}\right)$, the above discussion shows that quite a series 


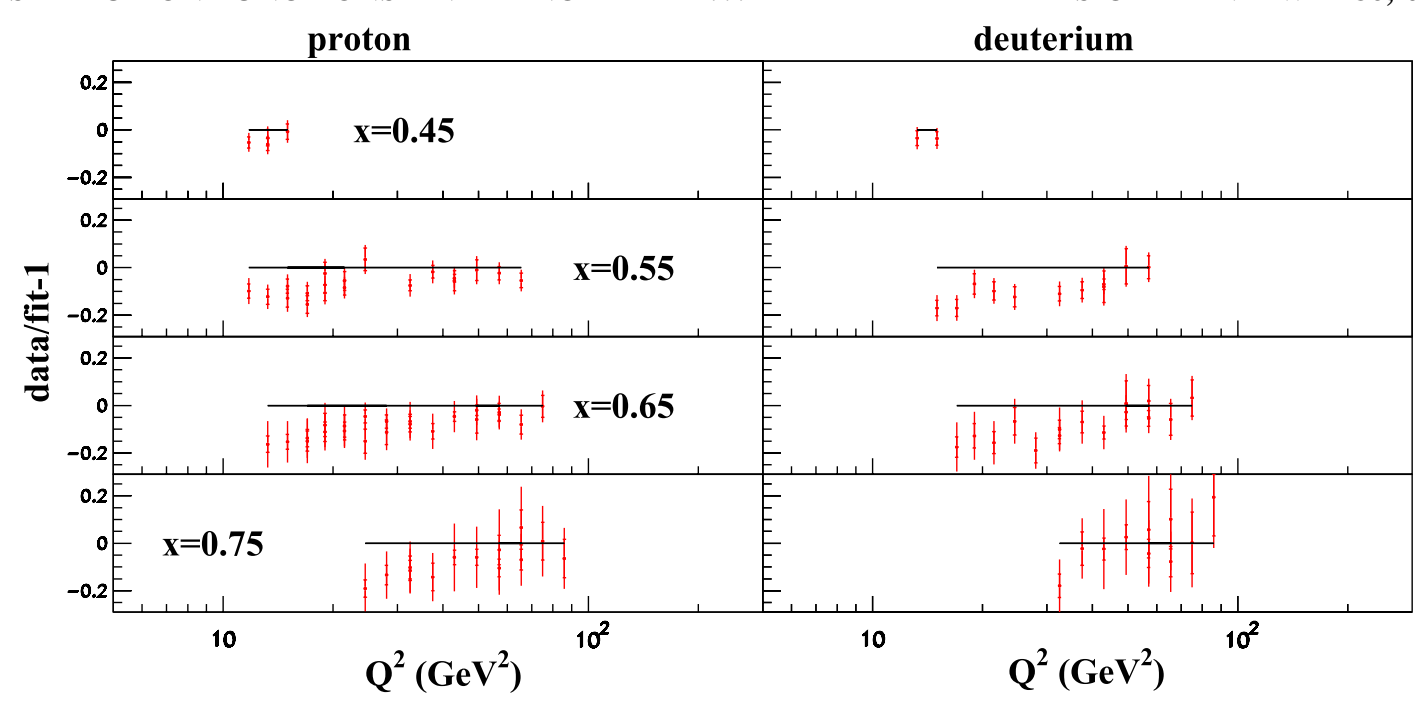

FIG. 21 (color online). Same as in Fig. 5 for the data points rejected in the analysis of Ref. [118].

of individual pulls are different. Neither ABM11 nor MSTW confirm the relatively large $\alpha_{s}\left(M_{Z}\right)$ values for the data sets of BCDMS and CDF R2KT and also the rise, at NNLO, of the NN21 value for CDF Zrap calls for further clarification; cf. Table IX.

Summarizing the comparison of the present results on $\alpha_{s}\left(M_{Z}\right)$ with the analyses [60,103,106,107,137,153] we observe a good agreement with Refs. [60,103] and find differences in a series of data sets for Refs. [106,107,137,153] both at NLO and NNLO, with partly different deviations in the case of Refs. [106,107,137,153]. NN21 does not agree with the BCDMS result. We find lower values of $\alpha_{s}\left(M_{Z}\right)$ both for the HERA and the SLAC data. We do not confirm part of the $\alpha_{s}\left(M_{Z}\right)$ values found in Refs. [106,107,137,153] for the jet data. We would like to mention once more that in our analysis no rescaling of errors is performed, which varies for different data sets in the analysis [107,153], but we

TABLE VII. Comparison of the values of $\alpha_{s}\left(M_{Z}\right)$ obtained by D0 in Ref. [114] with the ones based on including individual data sets of Tevatron jet data [108-111] into the analysis at NLO. The NNLO* fit refers to the NNLO analysis of the DIS and DY data together with the NLO and soft gluon resummation corrections (next-to-leading logarithmic accuracy) for the 1 jet inclusive data; cf. Refs. [102,112].

\begin{tabular}{lccc}
\hline \hline & & $\alpha_{s}\left(M_{Z}\right)$ & \\
Experiment & $\mathrm{NLO}_{\text {exp }}$ & NLO & NNLO* $^{*}$ \\
\hline D0 1 jet & $0.1161_{-0.0048}^{+0.0041}$ & $0.1190 \pm 0.0011$ & $0.1149 \pm 0.0012$ \\
D0 2 jet & & $0.1174 \pm 0.0009$ & $0.1145 \pm 0.0009$ \\
CDF 1 jet & & $0.1181 \pm 0.0009$ & $0.1134 \pm 0.0009$ \\
(cone) & & & \\
CDF 1 jet & $0.1181 \pm 0.0010$ & $0.1143 \pm 0.0009$ \\
$\left(k_{\perp}\right)$ & & & \\
ABM11 & & $0.1180 \pm 0.0012$ & $0.1134 \pm 0.0011$ \\
\hline \hline
\end{tabular}

have accounted for the systematic errors given by the experiments directly. The procedure of Refs. [107,153] naturally leads to reweighting of the impact of different data sets on the value of $\alpha_{s}\left(M_{Z}\right)$.

Finally we would like to summarize the results of different determinations of $\alpha_{s}\left(M_{Z}\right)$ at NNLO and $\mathrm{N}^{3} \mathrm{LO}$ (or $\mathrm{N}^{3} \mathrm{LO}^{*}$ ) in Table XII. Some part of these results has been reported in Ref. [129]. Flavor nonsinglet analyses of the DIS world data were performed in Refs. [60,103,122], with an accuracy of $\Delta \alpha_{s}\left(M_{Z}\right) \simeq 2 \%$ at NNLO. The difference between the value at $\mathrm{N}^{3} \mathrm{LO}^{*}$ and $\mathrm{NNLO}$ amounts to $\sim 0.0007$, which provides an estimate for the size of a remaining uncertainty. The ABKM09 analysis [16] is a combined flavor singlet/nonsinglet fit of the DIS world data, DY, and dimuon data. Here a remaining difference of $\Delta \alpha_{s}\left(M_{Z}\right)=0.0006$ due to the treatment of the heavyflavor corrections was observed. These uncertainties signal the typical theory errors remaining at the present level of description. The JR09 analysis obtained very similar results in combined flavor singlet/nonsinglet fits [20] with a slightly larger value in the standard fit compared to the dynamical approach. The present analysis obtains the same values as those found in Refs. $[16,20,60,103,122]$ before, while a slightly larger value $\alpha_{s}\left(M_{Z}\right)=0.1147 \pm 0.0012$ was reported in ABM10 [17] based on incorporating the combined HERA run I data [18]. However, the improved treatment of the heavy-quark contributions finally led to the present value $\alpha_{s}\left(M_{Z}\right)=0.1134 \pm 0.0011$. The inclusion of Tevatron jet data, cf. Ref. [102], although only a NNLO* analysis, alters this value at most to $\alpha_{s}\left(M_{Z}\right)=$ $0.1149 \pm 0.0012$ and the effect of the complete, yet unknown NNLO corrections, remains to be seen. Low values of $\alpha_{s}\left(M_{Z}\right)$ have not only been reported in analyses that are predominantly based on DIS data but also from those of thrust in $e^{+} e^{-}$-annihilation in Ref. [125]; cf. also Ref. [124]. 
TABLE VIII. Comparison of the values of $\alpha_{s}\left(M_{Z}\right)$ obtained by BCDMS [83] and NMC [105] at NLO with the results of the flavor nonsinglet fits BBG [60] and BB [103] of the DIS flavor nonsinglet world data, at NLO, NNLO, and $\mathrm{N}^{3} \mathrm{LO}^{*}$ with the response of the individual data sets, combined for the experiments BCDMS [83,119,120], NMC [84], and SLAC [121].

\begin{tabular}{lcccc}
\hline \hline & & \multicolumn{2}{c}{$\alpha_{s}\left(M_{Z}\right)$} & \\
Experiment & $\mathrm{NLO}_{\exp }$ & $\mathrm{NLO}$ & $\mathrm{NNLO}$ & $\mathrm{N}^{3} \mathrm{LO}^{*}$ \\
\hline BCDMS & $0.1111 \pm 0.0018$ & $0.1138 \pm 0.0007$ & $0.1126 \pm 0.0007$ & $0.1128 \pm 0.0006$ \\
NMC & $0.117_{-0.016}^{+0.011}$ & $0.1166 \pm 0.0039$ & $0.1153 \pm 0.0039$ & $0.1153 \pm 0.0035$ \\
SLAC & & $0.1147 \pm 0.0029$ & $0.1158 \pm 0.0033$ & $0.1152 \pm 0.0027$ \\
BBG & & $0.1148 \pm 0.0019$ & $0.1134 \pm 0.0020$ & $0.1141 \pm 0.0021$ \\
BB & & $0.1147 \pm 0.0021$ & $0.1132 \pm 0.0022$ & $0.1137 \pm 0.0022$ \\
\hline \hline
\end{tabular}

TABLE IX. Comparison of the values of $\alpha_{s}\left(M_{Z}\right)$ obtained by BCDMS [83], NMC [105], and D0 [114] at NLO with the results of NN21 [106,137] for the fits to DIS and other hard scattering data at NLO and NNLO and the corresponding response of the different data sets analyzed.

\begin{tabular}{lccc}
\hline \hline & & $\alpha_{s}\left(M_{Z}\right)$ & \\
Experiment & $\mathrm{NLO}_{\text {exp }}$ & $\mathrm{NLO}$ & $\mathrm{NNLO}$ \\
\hline BCDMS [82,83] $^{2}$ & $0.1111 \pm 0.0018$ & $0.1204 \pm 0.0015$ & $0.1158 \pm 0.0015$ \\
$\mathrm{NMC}_{p}[84]$ & & $0.1192 \pm 0.0018$ & $0.1150 \pm 0.0020$ \\
NMC $_{p d}[138]$ & & $0.1146 \pm 0.0107$ \\
SLAC [121] & & $>0.124$ & $>0.124$ \\
HERA I [18] & & $0.1223 \pm 0.0018$ & $0.1199 \pm 0.0019$ \\
ZEUS H2 [139,140] & $0.1170 \pm 0.0027$ & $0.1231 \pm 0.0030$ \\
ZEUS F2C [141-144] & $0.1144 \pm 0.0060$ & \\
NuTeV [95,96] & $0.1252 \pm 0.0068$ & $0.1177 \pm 0.0039$ \\
E605 [90] & & $0.1168 \pm 0.0100$ & \\
E866 [91,92,145] & $0.1135 \pm 0.0029$ & \\
CDF Wasy [146] & $0.1181 \pm 0.0060$ & \\
CDF Zrap [147] & & $0.1150 \pm 0.0034$ & $0.1205 \pm 0.0081$ \\
D0 Zrap [148] & & $0.1227 \pm 0.0067$ & \\
CDF R2KT [108] & & & \\
D0 R2CON [110] & & $0.1228 \pm 0.0021$ & $0.1225 \pm 0.0021$ \\
NN21 & & $0.1191 \pm 0.0031$ & $0.1111 \pm 0.0029$ \\
\hline \hline
\end{tabular}

Larger central values of $\alpha_{s}\left(M_{Z}\right)$ at NNLO (and similar in size) are reported by MSTW [107] and NN21 [106]. These fits include a much broader set of hadronic scattering data in the analysis, and above we have pointed out various differences between these analyses; see Tables IX and $\mathrm{X}$. These differences manifest themselves in various cases in rather different pulls for the value of $\alpha_{s}\left(M_{Z}\right)$. Note also that the $1 \sigma$ confidence level is very differently defined in the MSTW analysis compared to ABM11, NN21, and JR09. The (preliminary) central value of $\alpha_{s}\left(M_{Z}\right)$ reported by CT10 [123] is similar to MSTW and NN21 at NNLO, although accompanied by a rather large uncertainty of $\Delta \alpha_{s}\left(M_{Z}\right)=0.0050$, which makes the CT10 result compatible with the lower values obtained in Refs. [16,20,60,103,122]. We have also discussed the reasons for the difference between the central values of $\alpha_{s}\left(M_{Z}\right)$ in the NLO and NNLO analyses $[16,20,60,103,106,107,122]$ and further comparisons may be performed. An earlier claim that this is caused by the
Tevatron jet data is not confirmed; cf. Ref. [17]. Larger central values for $\alpha_{s}\left(M_{Z}\right)$ than in Refs. [16,20,60,103,122] are obtained for the 3-jet rate in $e^{+} e^{-}$annihilation [126] at NNLO and for the Z-decay width at $\mathrm{N}^{3} \mathrm{LO}$ [127]; see Table XII. The current $\alpha_{s}\left(M_{Z}\right)$ values at NNLO extracted from $\tau$ decays vary between 0.1212 and 0.1169 $[127,129,130]$. One lattice measurement [132] yields the same value as the current world average [129,134]. Other recent lattice results are compatible with this value and more lattice studies are still underway aiming at improved systematics [174].

\section{Heavy-quark masses}

The precise value of the heavy-quark masses is an important parameter in the description of DIS charm quark production. In our fit we use the heavy-quark masses in the $\overline{\mathrm{MS}}$ scheme and their implementation for heavy-quark DIS discussed in Ref. [16], which allows us to relate the values 
TABLE X. Comparison of the values of $\alpha_{s}\left(M_{Z}\right)$ obtained by BCDMS [83], NMC [105], HERA-jet [149,150] (see also Refs. [151,152]), and D0 [114] at NLO with the results of the MSTW fits to DIS and other hard scattering data at NLO and NNLO and the corresponding response of the different data sets analyzed; cf. Figs. 7a and 7b in Ref. [107]. Entries not given correspond to $\alpha_{s}\left(M_{Z}\right)$ central values below 0.110 or above 0.130 ; in case no errors are assigned, these are larger than the bounds provided in the form of the plots in Refs. [107,153].

\begin{tabular}{|c|c|c|c|}
\hline Experiment & $\mathrm{NLO}_{\exp }$ & $\begin{array}{c}\alpha_{s}\left(M_{Z}\right) \\
\mathrm{NLO}\end{array}$ & NNLO \\
\hline BCDMS $\mu p, F_{2}[82]$ & $0.1111 \pm 0.0018$ & $\cdots$ & $0.1085 \pm 0.0095$ \\
\hline BCDMS $\mu d, F_{2}[83]$ & & $0.1135 \pm 0.0155$ & $0.1117 \pm 0.0093$ \\
\hline $\mathrm{NMC} \mu p, F_{2}[84]$ & $0.117_{-0.016}^{+0.011}$ & $0.1275 \pm 0.0105$ & $0.1217 \pm 0.0077$ \\
\hline $\mathrm{NMC} \mu d, F_{2}[84]$ & & $0.1265 \pm 0.0115$ & $0.1215 \pm 0.0070$ \\
\hline $\mathrm{NMC} \mu n / \mu p[138]$ & & 0.1280 & 0.1160 \\
\hline $\mathrm{E} 665 \mu p, F_{2}[154]$ & & 0.1203 & $\cdots$ \\
\hline $\mathrm{E} 665 \mu d, F_{2}[154]$ & & $\cdots$ & $\cdots$ \\
\hline SLAC ep, $F_{2}[62,89]$ & & $0.1180 \pm 0.0060$ & $0.1140 \pm 0.0060$ \\
\hline SLAC $e d, F_{2}[62,89]$ & & $0.1270 \pm 0.0090$ & $0.1220 \pm 0.0060$ \\
\hline NMC, BCDMS, SLAC, $F_{L}[82,84,121]$ & & $0.1285 \pm 0.0115$ & $0.1200 \pm 0.0060$ \\
\hline E886/NuSea $p p$, DY [145] & & $\ldots$ & $0.1132 \pm 0.0088$ \\
\hline E886/NuSea $p d / p p$, DY [91] & & $0.1173 \pm 0.107$ & $0.1140 \pm 0.0110$ \\
\hline $\mathrm{NuTeV} \nu N, F_{2}[155]$ & & $0.1207 \pm 0.0067$ & $0.1170 \pm 0.0060$ \\
\hline CHORUS $\nu N, F_{2}[156]$ & & $0.1230 \pm 0.0110$ & $0.1150 \pm 0.0090$ \\
\hline $\mathrm{NuTeV} \nu N, x F_{3}[155]$ & & $0.1270 \pm 0.0090$ & $0.1225 \pm 0.0075$ \\
\hline CHORUS $\nu N, x F_{3}[156]$ & & $0.1215 \pm 0.0105$ & $0.1185 \pm 0.0075$ \\
\hline CCFR $[95,96]$ & & 0.1190 & $\cdots$ \\
\hline $\mathrm{NuTeV} \nu N \rightarrow \mu \mu X[95,96]$ & & $0.1150 \pm 0.0170$ & $\cdots$ \\
\hline $\mathrm{H} 1$ ep $97-00, \sigma_{r}^{\mathrm{NC}}[86,157-159]$ & & $0.1250 \pm 0.0070$ & $0.1205 \pm 0.0055$ \\
\hline ZEUS ep 95-00, $\sigma_{r}^{\mathrm{NC}}[87,160-162]$ & & $0.1235 \pm 0.0065$ & $0.1210 \pm 0.0060$ \\
\hline $\mathrm{H} 1$ ep $99-00, \sigma_{r}^{\mathrm{CC}}[158]$ & & $0.1285 \pm 0.0225$ & $0.1270 \pm 0.0200$ \\
\hline ZEUS ep 99-00, $\sigma_{r}^{\mathrm{CC}}$ & & $0.1125 \pm 0.0195$ & $0.1165 \pm 0.0095$ \\
\hline H1/ZEUS ep, $F_{2}^{\text {charm }}[141,142,164-167]$ & & $\ldots$ & $0.1165 \pm 0.0095$ \\
\hline H1 ep 99-00 incl. jets $[149,168]$ & $0.1168_{-0.0035}^{+0.0049}$ & $0.1127 \pm 0.0093$ & \\
\hline ZEUS ep 96-00 incl. jets $[150,169,170]$ & $0.1208_{-0.0040}^{+0.0044}$ & $0.1175 \pm 0.0055$ & \\
\hline D0 II $p \bar{p}$ incl. jets $[110]$ & $0.1161_{-0.0048}^{+0.0041}$ & $0.1185 \pm 0.0055$ & $0.1133 \pm 0.0063$ \\
\hline CDF II $p \bar{p}$ incl. jets [108] & & $0.1205 \pm 0.0045$ & $0.1165 \pm 0.0025$ \\
\hline D0 II $W \rightarrow l \nu$ asym. [171] & & $\cdots$ & $\cdots$ \\
\hline CDF II $W \rightarrow l \nu$ asym. [172] & & $\ldots$ & $\ldots$ \\
\hline D0 II $Z$ rap. [148] & & $0.1125 \pm 0.0100$ & $0.1136 \pm 0.0084$ \\
\hline CDF II $Z$ rap. [173] & & $0.1160 \pm 0.0070$ & $0.1157 \pm 0.0067$ \\
\hline MSTW & & $0.1202_{-0.0015}^{+0.0012}$ & $0.1171 \pm 0.0014$ \\
\hline
\end{tabular}

TABLE XI. Comparison of the pulls in $\alpha_{s}\left(M_{Z}\right)$ per data set between the ABM11 as shown in Fig. 19, BBG [60], NN21 [106], and MSTW [107] analyses at NNLO. The values in parentheses of ABM11 correspond to Fig. 18 where the shape parameters are not refitted, which is also the case for BBG.

\begin{tabular}{lcccc}
\hline \hline Data set & ABM11 & BBG & NN21 & MSTW \\
\hline BCDMS & $0.1128 \pm 0.0020$ & $0.1126 \pm 0.0007$ & $0.1158 \pm 0.0015$ & $0.1101 \pm 0.0094$ \\
& $(0.1084 \pm 0.0013)$ & & & \\
NMC & $0.1055 \pm 0.0026$ & $0.1153 \pm 0.0039$ & $0.1150 \pm 0.0020$ & $0.1216 \pm 0.0074$ \\
& $(0.1152 \pm 0.0007)$ & & & \\
SLAC & $0.1184 \pm 0.0021$ & $0.1158 \pm 0.0034$ & $>0.124$ & $0.1140 \pm 0.0060 \mathrm{ep}$ \\
& $(0.1128 \pm 0.0003)$ & & $0.1199 \pm 0.0019$ & $0.1208 \pm 0.0058$ \\
HERA & $0.1139 \pm 0.0014$ & & $0.1231 \pm 0.0030$ & \\
& $(0.1126 \pm 0.0002)$ & & - & $0.1136 \pm 0.0100$ \\
DY & $(0.101 \pm 0.025)$ & - & $0.1173 \pm 0.0007$ & $0.1171 \pm 0.0014$ \\
\hline \hline
\end{tabular}


TABLE XII. Summary of recent NNLO QCD analyses of the DIS world data, supplemented by related measurements using other processes.

\begin{tabular}{|c|c|c|}
\hline & $\alpha_{s}\left(M_{Z}\right)$ & \\
\hline BBG & $0.1134_{-0.0021}^{+0.0019}$ & valence analysis, NNLO [60] \\
\hline BB & $0.1132 \pm 0.0022$ & valence analysis, NNLO [103] \\
\hline GRS & 0.112 & valence analysis, NNLO [122] \\
\hline ABKM & $0.1135 \pm 0.0014$ & HQ: FFNS $n_{f}=3[16]$ \\
\hline ABKM & $0.1129 \pm 0.0014$ & HQ: BSMN-approach [16] \\
\hline $\mathrm{JR}$ & $0.1124 \pm 0.0020$ & dynamical approach [20] \\
\hline JR & $0.1158 \pm 0.0035$ & standard fit [20] \\
\hline ABM11 & $0.1134 \pm 0.0011$ & \\
\hline MSTW & $0.1171 \pm 0.0014$ & [107] \\
\hline NN21 & $0.1173 \pm 0.0007$ & {$[106]$} \\
\hline CT10 & $0.118 \pm 0.005$ & {$[123]$} \\
\hline Gehrmann et al. & $0.1153 \pm 0.0017 \pm 0.0023$ & $e^{+} e^{-}$thrust $[124]$ \\
\hline Abbate et al. & $0.1135 \pm 0.0011 \pm 0.0006$ & $e^{+} e^{-}$thrust [125] \\
\hline 3 jet rate & $0.1175 \pm 0.0025$ & Dissertori et al. 2009 [126] \\
\hline Z-decay & $0.1189 \pm 0.0026$ & BCK $2008 / 12\left(\mathrm{~N}^{3} \mathrm{LO}\right)[127,128]$ \\
\hline$\tau$ decay & $0.1212 \pm 0.0019$ & BCK 2008 [127] \\
\hline$\tau$ decay & $0.1204 \pm 0.0016$ & Pich 2011 in Ref. [129] \\
\hline$\tau$ decay & $0.1169 \pm 0.0025$ & Boito et al. 2011 [130] \\
\hline Lattice & $0.1205 \pm 0.0010$ & PACS-CS 2009 (2 + 1 fl. $)$ [131] \\
\hline Lattice & $0.1184 \pm 0.0006$ & HPQCD 2010 [132] \\
\hline Lattice & $0.1200 \pm 0.0014$ & ETM $2012(2+1+1$ fl. $)$ [133] \\
\hline BBG & $0.1141_{-0.0022}^{+0.0020}$ & valence analysis, $\mathrm{N}^{3} \mathrm{LO}(*)[60]$ \\
\hline $\mathrm{BB}$ & $0.1137 \pm 0.0022$ & valence analysis, $\mathrm{N}^{3} \mathrm{LO}(*)[103]$ \\
\hline \multirow[t]{2}{*}{ World average } & $0.1184 \pm 0.0007$ & {$[134](2009)$} \\
\hline & $0.1183 \pm 0.0010$ & [129] (2011) \\
\hline
\end{tabular}

for $m_{c}$ and $m_{b}$ directly to PDG results [98], as done in Eq. (4.10).

The current DIS data displays great sensitivity to the charm mass $m_{c}$ as we have demonstrated previously [32,175]. Therefore, based on the pseudodata input from Eq. (4.10) we have released the uncertainty of the quark masses to obtain the following results:

$$
\begin{aligned}
& m_{c}\left(m_{c}\right)=1.27 \pm 0.06 \mathrm{GeV} \\
& m_{b}\left(m_{b}\right)=4.19 \pm 0.13 \mathrm{GeV}
\end{aligned}
$$

which shows that the error on $m_{c}$ from the inclusive DIS data used in the fit is comparable to the one quoted by the PDG [98] (although not comparable to the single most precise measurement listed therein). Interestingly, we observe in the covariance matrix in Tables XXVI, XXVII, and XXVIII correlations of $m_{c}$ with $\alpha_{s}$ and some parameters of the gluon and the strange PDFs. The precision of DIS data to the value of $m_{c}$ has previously also been exploited for the first direct determination of the running mass for charm quarks from hadronic processes with spacelike kinematics as a variant of ABKM09 yielding values consistent with but systematically somewhat lower than the PDG world average [32,175].
It is interesting to compare the results of the fit to the most recent HERA data from the H1 Collaboration [176] for the charm structure function $F_{2}^{c}$ in heavy-quark DIS extracted with the use of the HVQDIS code [177]. This is done in Fig. 22 for our 3-flavor running-mass NNLO predictions with $m_{c}$ of Eq. (4.15) using the approximate NNLO QCD predictions of Refs. [39-41]. The data are not used in our fit; however, the agreement is quite good, as well as for the predictions based on the NNLO 3-flavor PDFs of JR09 [20] and MSTW [22]. The predictions using the NLO 3-flavor NN21 PDFs of Ref. [100] undershoot the data. The differences may be related to the peculiarities of the so-called general mass VFNS modeling employed in those fits [22,24,100]. Also wrong analyses of the combined $\mathrm{H} 1$ and ZEUS data on $F_{2}^{c}$ exist [178].

Finally, we want to mention that the issue of heavyquark masses also has consequences for heavy-quark PDFs, because the uncertainty on heavy-quark PDFs is directly related to the accuracy of the numerical values for the quark masses $m_{c}$ or $m_{b}$. The latter appear parametrically in the OMEs used to generate charm and bottom PDFs in schemes with $n_{f}=4$ or $n_{f}=5$ flavors. With the results of Eq. (4.15) and the use of the $\overline{\mathrm{MS}}$ scheme, this uncertainty in the charm and bottom PDFs can be significantly reduced. 


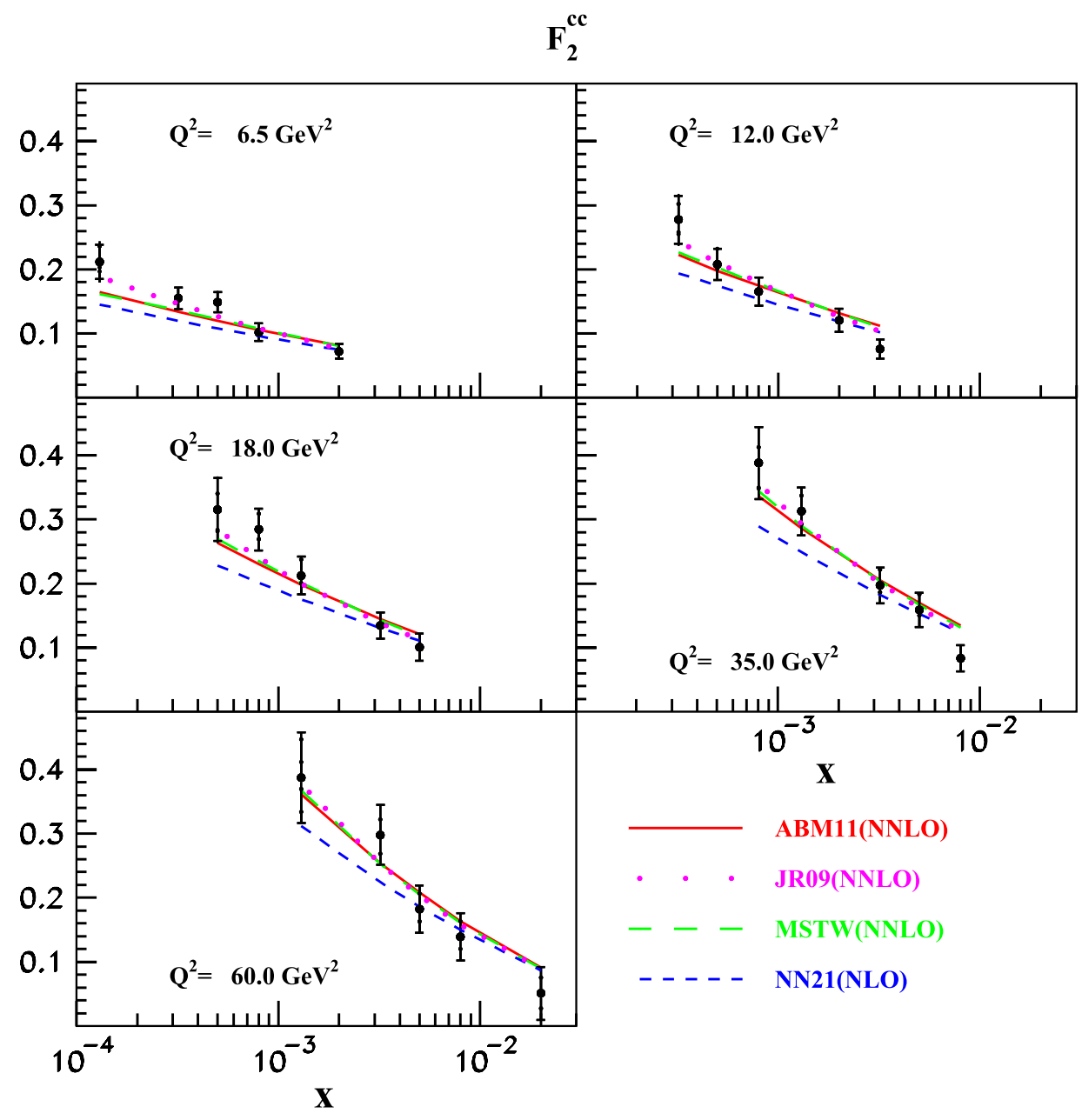

FIG. 22 (color online). Comparison of the data from Ref. [176] for the semi-inclusive structure function $F_{2}^{c c}$ at different values of the momentum transfer $Q^{2}$ vs $x$ with predictions of various PDF sets at NLO and NNLO in QCD, all taken in the FFNS with $n_{f}=3$ and with a running mass of $m_{c}=1.27 \mathrm{GeV}$ [98]. NNLO predictions for $F_{2}^{c c}$ use the ABM11 PDFs (solid curves), the JR09 PDFs [20] (dotted lines), and the MSTW PDFs of Ref. [22] (long dashed lines). NLO calculations are based on the NN21 PDFs of Ref. [100] (short dashed lines).

In Fig. 23 we display the PDFs generated in this way. We obtain a charm PDF with comparable uncertainties to the one of Ref. [16] (which then has used the pole mass definition for $m_{c}$ ), while the resulting uncertainty of the bottom PDF is greatly reduced; see also Ref. [175]. This improvement has a significant impact on LHC phenomenology, as it will, e.g., allow for precise predictions for the production of single-top quarks and other processes sensitive to bottom PDFs.

\section{Moments of the PDFs}

In Table XIII we summarize different values of the second moment of the valence quark densities obtained in NNLO analyses at the scale $Q^{2}=4 \mathrm{GeV}^{2}$. It is evident that these moments are rather stable quantities for all PDF sets considered as they are mostly influenced by the data normalization. They are closely related to the moments that are being measured in lattice simulations. Of central importance is the quantity

$$
\begin{aligned}
\left\langle x V\left(Q^{2}\right)\right\rangle= & \int_{0}^{1} d x x\left\{\left[u\left(x, Q^{2}\right)+\bar{u}_{s}\left(x, Q^{2}\right)\right]\right. \\
& \left.-\left[d\left(x, Q^{2}\right)+\bar{d}_{s}\left(x, Q^{2}\right)\right]\right\},
\end{aligned}
$$

where $q \equiv q_{v}+q_{s}$ with $q=u, d$.

In Fig. 24 the result for Eq. (4.16) obtained in the present analysis is compared with recent lattice computations using varying numbers of flavors as a function of the pion mass $m_{\pi}$ employed on the lattice. In detail, these are QCDSF $\left(n_{f}=2\right)$ [179], RBC/UKQCD $\left(n_{f}=2+1\right)$ [180], LHPC $\left(n_{f}=2+1\right)$ [181], ETMC $\left(n_{f}=2\right)$ [182], and ETMC $\left(n_{f}=2+1+1\right)$ [183,184]. It is apparent from Fig. 24 that there are substantial differences, even for low pion masses, between those lattice measurements 

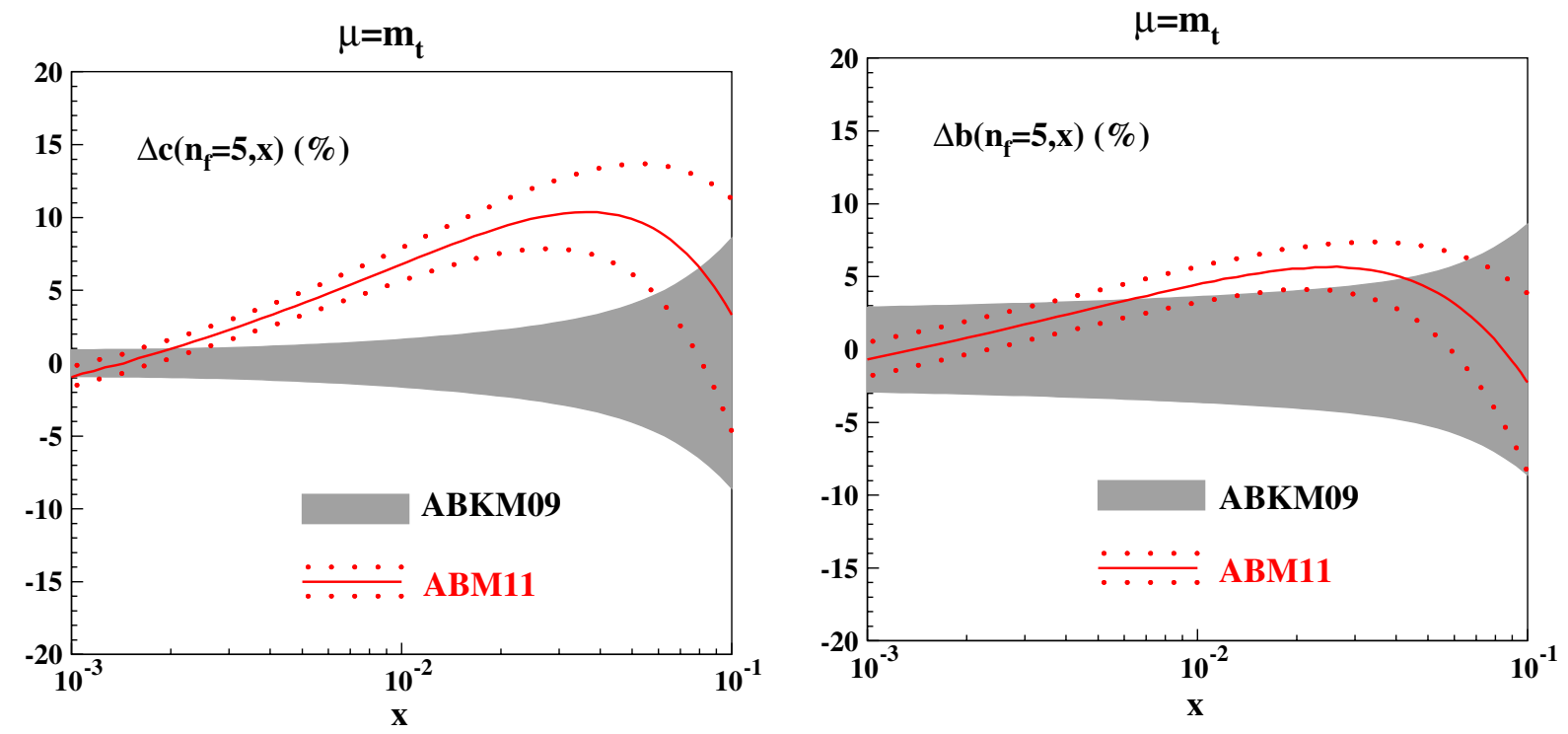

FIG. 23 (color online). Charm (left) and the bottom-quark (right) PDFs obtained in the global fit: Dotted (red) lines denote the $\pm 1 \sigma$ band of relative uncertainties (in percent) and solid (red) lines indicate the central prediction resulting from the fit with the running masses of Eq. (4.15). For comparison the shaded (gray) area represents the results of ABKM09 [16].

and the experimental determinations of Table XIII. For very recent progress see Ref. [185].

\section{BENCHMARKS FOR CROSS SECTIONS}

In this section we quantify the impact of the new PDF set on predictions for benchmark cross sections at the Tevatron and the LHC. To that end, we confine ourselves to (mostly) inclusive cross sections that are known to NNLO in QCD either completely or in very good approximation; see Ref. [26] for previous work along these lines. NNLO accuracy is actually the first instance, where meaningful statements about the residual theoretical uncertainty are possible, since at NLO the latter that is conventionally determined from a variation of the renormalization and factorization scale is generally still too large, given the precision of present collider data.

In detail, we consider the following set of inclusive observables: hadronic $W$ and $Z$ boson production [11,12], the cross section for Higgs boson production in the dominant channels, ggF [12,186-188], vector-boson fusion (VBF) with VBFNNLO [189,190], and in Higgs-strahlung [191]. The cross section for top-quark pair production is approximately NNLO (based on threshold resummation, see e.g., Ref. [192]) and is computed with HATHOR (version 1.2) [193]. We also consider the lepton $\left(l^{ \pm}\right)$charge asymmetry in hadronic $W^{ \pm}$boson production as a function of the rapidity [13-15,194]. Throughout the entire section we focus on the QCD corrections only. That is to say, we neglect all electroweak radiative effects at NLO, which often amount to corrections of $\mathcal{O}(\mathrm{few}) \%$ at the $\mathrm{LHC}$ and, therefore, need to be considered in precision predictions.

The PDF uncertainties quoted here are calculated by summing over the $n_{\mathrm{PDF}}$ sets provided by the various groups, where $n_{\mathrm{PDF}}$ is the number of parameters used in the fit. Typically, we quote the symmetric error according to

$$
\Delta \sigma_{\mathrm{PDF}}=\sqrt{\sum_{k=1, n_{\mathrm{PDF}}}\left(\sigma_{0}-\sigma_{k}\right)^{2}}
$$

TABLE XIII. Comparison of the second moment of the valence quark distributions at NNLO and $\mathrm{N}^{3} \mathrm{LO}$ obtained in different analyses at $Q^{2}=4 \mathrm{GeV}^{2}$.

\begin{tabular}{lllll}
\hline \hline & \multicolumn{1}{c}{$\left\langle x u_{v}(x)\right\rangle$} & \multicolumn{1}{c}{$\left\langle x d_{v}(x)\right\rangle$} & \multicolumn{1}{c}{$\left\langle x\left[u_{v}-d_{v}\right](x)\right\rangle$} & $\langle x V(x)\rangle$ \\
\hline ABM11 & $0.2971 \pm 0.0039$ & $0.1174 \pm 0.0050$ & $0.1797 \pm 0.0042$ & $0.1655 \pm 0.0039$ \\
ABKM09 [16] & $0.2981 \pm 0.0025$ & $0.1191 \pm 0.0023$ & $0.1790 \pm 0.0023$ & $0.1647 \pm 0.0022$ \\
HERAPDF1.5 [18,19] & $0.2938_{-0.0052}^{+0.0031}$ & $0.1264_{-0.0059}^{+0.0054}$ & $0.1674_{-0.0052}^{+0.0043}$ & $0.1706_{-0.0103}^{+0.0071}$ \\
JR09 [20,21] & $0.2897 \pm 0.0035$ & $0.1253 \pm 0.0052$ & $0.1645 \pm 0.0063$ & $0.1513 \pm 0.0118$ \\
MSTW [22] & $0.2816_{-0.0042}^{+0.0051}$ & $0.1171_{-0.0028}^{+0.0027}$ & $0.1645_{-0.0034}^{+0.0046}$ & $0.1533_{-0.0033}^{+0.0041}$ \\
NN21 [23] & $0.2913 \pm 0.0038$ & $0.1218 \pm 0.0042$ & $0.1695 \pm 0.0040$ & $0.1539 \pm 0.0030$ \\
BBG [60] & $0.2986 \pm 0.0029$ & $0.1239 \pm 0.0026$ & $0.1747 \pm 0.0039$ & \\
BBG [N LO] [60] & $0.3006 \pm 0.0031$ & $0.1252 \pm 0.0027$ & $0.1754 \pm 0.0041$ & \\
\hline \hline
\end{tabular}




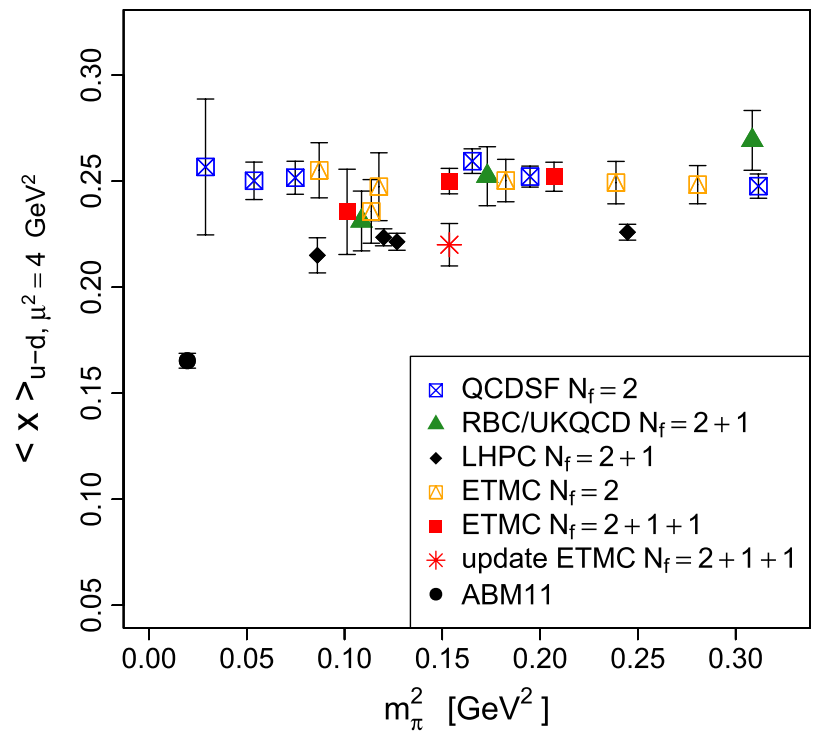

FIG. 24 (color online). Comparison of lattice computations for the second moment of the nonsinglet distribution as a function of the pion mass $m_{\pi}$ with the result of ABM11 given in Table XIII along with the uncertainties of the respective measurement.

where $\sigma_{k}$ is obtained by using the $k$ th PDF $f_{i}^{k}$, which parametrizes the $\pm 1 \sigma$ variation of the $k$ th fit parameter after diagonalization of the correlation matrix. In some cases, e.g., for MSTW [22], asymmetric PDF errors are provided, in which case the variation in the $k$ th fit parameter is given by a pair of PDFs $f_{i}^{k, \pm}$. The resulting asymmetric PDF error is then computed according to

$$
\Delta \sigma_{\mathrm{PDF}}^{+}=\sqrt{\sum_{k=1, n_{\mathrm{PDF}}} \max \left(0,+\sigma_{k,+}-\sigma_{0},+\sigma_{k,-}-\sigma_{0}\right)^{2}},
$$

$$
\Delta \sigma_{\mathrm{PDF}}^{-}=\sqrt{\sum_{k=1, n_{\mathrm{PDF}}} \min \left(0,-\sigma_{k,+}+\sigma_{0},-\sigma_{k,-}+\sigma_{0}\right)^{2}}
$$

For MSTW, we are using the set with $68 \%$ confidence level error estimates throughout.
In a Monte Carlo approach like the one advocated by NN21 [23], the PDF uncertainty can be determined as the quadratic deviation from the central fit as in Eq. (5.1), but with an additional factor $1 / \sqrt{n_{\mathrm{PDF}}}$. For reasons of efficiency and run times, we are using the NN21 PDF with 100 sets in our comparisons only; see also the discussion in Sec. VE.

\section{A. $W$ and $Z$ boson production}

We start by presenting results for $W$ and $Z$ boson production at the LHC at $\sqrt{s}=7 \mathrm{TeV}$. For the electroweak parameters, we follow Ref. [26] and choose the scheme based on the set $\left(G_{F}, M_{W}, M_{Z}\right)$. According to Ref. [98], we have $G_{F}=1.16637 \times 10^{-5} \mathrm{GeV}^{-2}, M_{W}=80.399 \pm$ $0.023 \mathrm{GeV}, M_{Z}=91.1876 \pm 0.0021 \mathrm{GeV}$, and the corresponding widths $\Gamma\left(W^{ \pm}\right)=2.085 \pm 0.042 \mathrm{GeV}$ and $\Gamma(Z)=2.4952 \pm 0.0023 \mathrm{GeV}$. The weak mixing angle is then a dependent quantity, with

$$
\hat{s}_{Z}^{2}=1-\frac{M_{W}^{2}}{\hat{\rho} M_{Z}^{2}}=0.2307 \pm 0.0005,
$$

and $\hat{\rho}=1.01047 \pm 0.00015$. Finally, the Cabibbo angle $\theta_{c}$ yields the value $\sin ^{2} \theta_{c}=0.051$.

At NNLO the theoretical uncertainty due to scale variation is small compared to the PDF error; see Tables XIV and XV. The change in the predictions between ABKM09 and ABM11 is small. NN21 and MSTW typically predict smaller cross sections with differences at the level of $1-2 \sigma$, while the numbers of JR09 are significantly smaller; see also the detailed discussion in Ref. [26]. Most importantly, there is the choice to consider, in particular, the $W$ boson cross section in alternative schemes with $n_{f}=4$ or $n_{f}=5$ flavors, since contributions of the initial bottom PDFs being proportional to the CKM matrix element $V_{t b}$ are kinematically suppressed.

Comparing the results in Tables XIV and XV for the PDF sets with $n_{f}=4$ and $n_{f}=5$, we observe that the numbers for the $n_{f}=5$ scheme are always larger, the differences being less than $1 \sigma$ in the PDF uncertainty, though. These differences, which become successively smaller at higher orders, i.e., as we go from NLO to NNLO accuracy, originate from changes in the light flavor

TABLE XIV. Total cross sections for gauge boson production at the $\mathrm{LHC}(\sqrt{s}=7 \mathrm{TeV})$ for different PDF sets and to NNLO accuracy The errors shown are the scale uncertainty based on the shifts $\mu=M_{W / Z} / 2$ and $\mu=2 M_{W / Z}$ and, respectively, the $1 \sigma$ PDF uncertainty. Numbers are in $\mathrm{pb}$.

\begin{tabular}{lccccc}
\hline \hline & ABM11 & ABKM09 [16] & JR09 [20,21] & MSTW [22] & NN21 [23] \\
\hline$W^{+}$ & $59.53_{-0.23-0.88}^{+0.38+0.88}$ & $59.30_{-0.24-0.93}^{+0.39+0.93}$ & $54.68_{-0.19-1.30}^{+0.32+1.30}$ & $57.20_{-0.14-0.95}^{+0.31+1.02}$ & $58.46_{-0.21-0.91}^{+0.35+0.91}$ \\
$W^{-}$ & $39.97_{-0.17-0.65}^{+0.28+0.65}$ & $39.70_{-0.18-0.63}^{+0.28+0.63}$ & $37.22_{-0.14-0.92}^{+0.24+0.92}$ & $39.89_{-0.12-0.67}^{+0.24+0.69}$ & $39.75_{-0.17-0.63}^{+0.27+0.63}$ \\
$W^{ \pm}$ & $99.51_{-0.41-1.43}^{+0.69+1.43}$ & $99.00_{-0.41-1.53}^{+0.67+1.53}$ & $91.91_{-0.34-2.14}^{+0.55+2.14}$ & $97.10_{-0.27-1.57}^{+0.53+1.66}$ & $98.21_{-0.38-1.40}^{+0.62+1.40}$ \\
$Z$ & $29.23_{-0.10-0.42}^{+0.18+0.42}$ & $29.08_{-0.10-0.46}^{+0.18+0.46}$ & $26.90_{-0.08-0.58}^{+0.15+0.58}$ & $28.58_{-0.07-0.46}^{+0.14+0.49}$ & $28.71_{-0.09-0.38}^{+0.17+0.38}$ \\
\hline \hline
\end{tabular}


TABLE XV. Same as Table XIV for the PDF sets with $n_{f}=4$.

\begin{tabular}{lccc}
\hline \hline & ABM11 & ABKM09 [16] & MSTW [22] \\
\hline$W^{+}$ & $59.08_{-0.14-0.87}^{+0.30+0.87}$ & $58.85_{-0.15-0.92}^{+0.31+0.92}$ & $56.77_{-0.08-0.94}^{+0.24+1.01}$ \\
$W^{-}$ & $39.70_{-0.12-0.64}^{+0.22+0.64}$ & $39.43_{-0.12-0.62}^{+0.22+0.62}$ & $39.61_{-0.08-0.66}^{+0.19+0.69}$ \\
$W^{ \pm}$ & $98.77_{-0.25-1.41}^{+0.53+1.41}$ & $98.28_{-0.27-1.51}^{+0.53+1.51}$ & $96.38_{-0.16-1.56}^{+0.43+1.65}$ \\
$Z$ & $28.54_{-0.05-0.42}^{+0.13+0.42}$ & $28.44_{-0.06-0.45}^{+0.12+0.45}$ & $27.91_{-0.03-0.46}^{+0.09+0.50}$ \\
\hline \hline
\end{tabular}

and the gluon PDFs when the bottom PDF is generated perturbatively; recall Sec. IV C. In summary, the differences between the results in Tables XIV and XV for a given PDF set constitute an intrinsic uncertainty of the perturbative prediction. Comparisons of heavy-flavor PDFs including mass effects have also been studied in Ref. [195].

Next, we address the charged-lepton asymmetry data $[196,197]$ as obtained by the ATLAS and CMS experiments and compare it to the NNLO predictions based on the ABM11 PDFs in Fig. 25. All differential distributions for $W$ and $Z$ boson production are computed with the fully exclusive NNLO program DYNNLO $[15,194]$, which allows one to take into account the kinematical cuts imposed in the experiments (cf. Fig. 25); see also Ref. [198] for an alternative code.

The overall agreement with both experiments is sufficiently good; however, at values of $\eta \sim 1.5$ for the lepton pseudorapidity the data show a different trend with respect to the predictions. Preliminary data on the charge-lepton asymmetry at large rapidities obtained by the $\mathrm{LHCb}$ Collaboration [199] are also in good agreement with the
ABKM09 predictions. To check the impact of the LHC charged-lepton asymmetry data on our fit, we have performed a variant of the ABM11 analysis that consists of adding the data of Refs. [196,197]. We have found, however, that the change in the PDF central values and their errors is only marginal in view of still big uncertainties in the data.

\section{B. Higgs boson production}

Let us now discuss the cross sections for the standard model Higgs boson production, where all dominant channels are known to NNLO in QCD.

We start with ggF in Tables XVI, XVII, and XVIII, where the cross section is driven by the gluon luminosity and the value of $\alpha_{s}$ from the effective vertex. The NNLO QCD corrections obtained in Refs. [12,186-188] still lead to a sizable increase in the cross section at nominal values of the scale, i.e., $\mu=m_{H}$. Further stabilization is achieved beyond NNLO on the basis of soft gluon resummation; see e.g., Ref. [200].

We observe in Tables XVI, XVII, and XVIII that the ABM11 predictions are rather stable with small changes only due to the gluon PDF discussed in Sec. IV. The values of MSTW are typically larger than those of ABM11 and of ABKM09, roughly by $\mathcal{O}(10 \%)$ depending on the Higgs mass and the LHC collision energy, which has direct consequences for the current Higgs searches at the LHC. For example, at $m_{H}=125 \mathrm{GeV}$ MSTW predicts an $8 \%$ larger cross section of which $6.5 \%$ are due to the difference in $\alpha_{s}$. In terms of PDF uncertainties, this discrepancy is
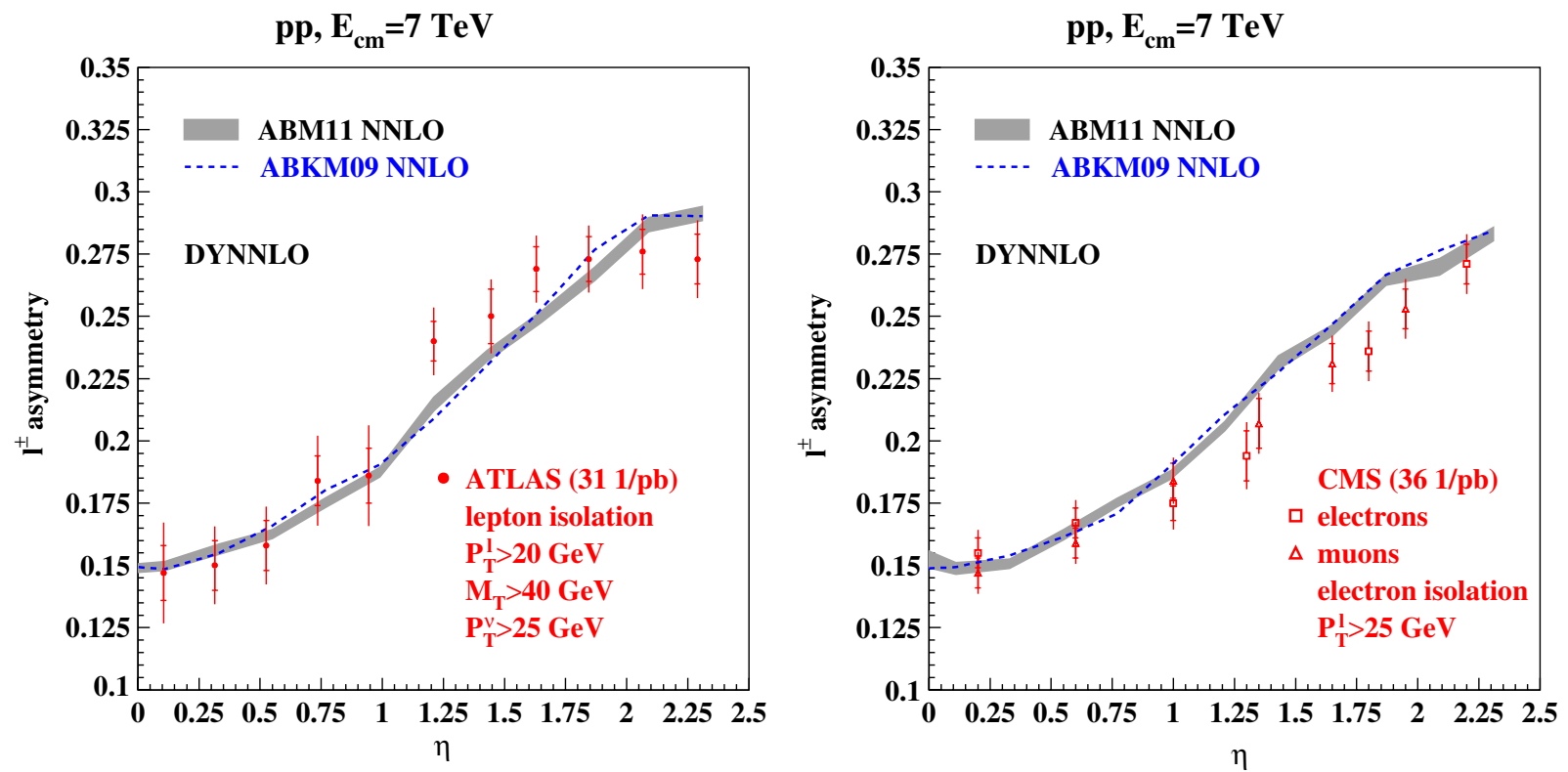

FIG. 25 (color online). Data on charged-lepton asymmetry vs the lepton pseudorapidity $\eta$ obtained by the ATLAS [196] (left) and CMS [197] (right) experiments compared to the NNLO predictions based on the DYNNLO code [15,194] and the ABM11 NNLO PDFs with the shaded area showing the integration uncertainties. ABKM09 NNLO predictions are given for comparison by dashed lines, without the integration uncertainties shown. 
TABLE XVI. Total cross sections for Higgs production in $\mathrm{ggF}$ at the $\mathrm{LHC}(\sqrt{s}=7 \mathrm{TeV})$ for different PDF sets and to NNLO accuracy. Errors shown are the scale uncertainty based on the shifts $\mu=m_{H} / 2$ and $\mu=2 m_{H}$ and, respectively, the $1 \sigma$ PDF uncertainty. Numbers are in pb.

\begin{tabular}{|c|c|c|c|c|c|}
\hline$m_{H}$ & ABM11 & ABKM09 [16] & JR09 [20,21] & MSTW [22] & NN21 [23] \\
\hline 100 & $21.31_{-2.10-0.46}^{+2.20+0.46}$ & $21.19_{-2.11-0.60}^{+2.21+0.60}$ & $20.47_{-1.87-0.70}^{+1.99+0.70}$ & $22.95_{-2.34-0.35}^{+2.50+0.25}$ & $24.16_{-2.49-0.31}^{+2.73+0.31}$ \\
\hline 110 & $17.43_{-1.73-0.38}^{+1.79+0.38}$ & $17.31_{-1.72-0.49}^{+1.79+0.49}$ & $16.91_{-1.54-0.56}^{+1.63+0.56}$ & $18.84_{-1.92-0.30}^{+2.03+0.20}$ & $19.83_{-2.02-0.27}^{+2.22+0.27}$ \\
\hline 115 & $15.85_{-1.57-0.34}^{+1.62+0.34}$ & $15.73_{-1.57-0.45}^{+1.62+0.45}$ & $15.45_{-1.40-0.50}^{+1.48+0.50}$ & $17.17_{-1.75-0.26}^{+1.85+0.20}$ & $18.07_{-1.84-0.25}^{+2.01+0.25}$ \\
\hline 120 & $14.46_{-1.43-0.32}^{+1.48+0.32}$ & $14.34_{-1.43-0.42}^{+1.47+0.42}$ & $14.18_{-1.28-0.45}^{+1.35+0.45}$ & $15.70_{-1.60-0.25}^{+1.68+0.17}$ & $16.51_{-1.67-0.23}^{+1.84+0.23}$ \\
\hline 125 & $13.23_{-1.31-0.30}^{+1.35+0.30}$ & $13.12_{-1.31-0.38}^{+1.34+0.38}$ & $13.02_{-1.17-0.41}^{+1.24+0.41}$ & $14.39_{-1.47-0.22}^{+1.54+0.17}$ & $15.14_{-1.53-0.21}^{+1.68+0.21}$ \\
\hline 130 & $12.14_{-1.21-0.28}^{+1.23+0.28}$ & $12.04_{-1.20-0.35}^{+1.23+0.35}$ & $12.01_{-1.07-0.37}^{+1.14+0.37}$ & $13.24_{-1.35}^{+1.40}$ & $13.92_{-1.40-0.20}^{+1.54+0.20}$ \\
\hline 140 & $10.30_{-1.03-0.24}^{+1.04+0.24}$ & $10.21_{-1.02-0.31}^{+1.03+0.31}$ & $10.28_{-0.92-0.32}^{+0.97+0.32}$ & $11.28_{-1.16-0.18}^{+1.19+0.14}$ & $11.86_{-1.19-0.18}^{+1.30+0.18}$ \\
\hline 150 & $8.83_{-0.88-0.21}^{+0.89+0.21}$ & $8.75_{-0.87-0.27}^{+0.88+0.27}$ & $8.90_{-0.79-0.27}^{+0.83+0.27}$ & $9.71_{-0.99-0.16}^{+1.02+0.13}$ & $10.21_{-1.02-0.16}^{+1.11+0.16}$ \\
\hline 160 & $7.63_{-0.77-0.19}^{+0.76+0.19}$ & $7.57_{-0.76-0.24}^{+0.76+0.24}$ & $7.76_{-0.6}^{+0.7}$ & $8.44_{-0.8}^{+0.8}$ & $8.86_{-0.88-0.14}^{+0.96+0.14}$ \\
\hline 180 & $5.84_{-0.59-0.15}^{+0.58+0.15}$ & $5.79_{-0.58}^{+0.57}$ & $6.04_{-0}^{+0}$ & $6.51_{-0}^{+0}$ & $6.83_{-0.67-0.12}^{+0.73+0.12}$ \\
\hline 200 & $4.58_{-0.46-0.13}^{+0.45+0.13}$ & $4.55_{-0.46-0.16}^{+0.45+0.16}$ & $4.83_{-0.42}^{+0.44}$ & $5.17_{-0 .}^{+0}$ & $5.42_{-0.54-0.10}^{+0.57+0.10}$ \\
\hline 220 & $3.69_{-0.37-0.11}^{+0.36+0.11}$ & $3.67_{-0.37-0.14}^{+0.36+0.14}$ & $3.97_{-0.35-0.14}^{+0.36+0.14}$ & $4.20_{-0.43-0.08}^{+0.43+0.07}$ & $4.41_{-0.44-0.08}^{+0.45+0.08}$ \\
\hline 260 & $2.55_{-0.26-0.08}^{+0.25+0.08}$ & $2.55_{-0.26-0.10}^{+0.25+0.10}$ & $2.85_{-0.25-0.12}^{+0.25+0.12}$ & $2.97_{-0.31-0.07}^{+0.30+0.06}$ & $3.11_{-0.31-0.07}^{+0.31+0.07}$ \\
\hline 300 & $1.93_{-0.20-0.07}^{+0.19+0.07}$ & $\begin{array}{r}1.95_{-0.20-0.09}^{+0.19+0.09} \\
\end{array}$ & $2.23_{-0.20-0.11}^{+0.19+0.11}$ & $2.30_{-0.24-0.06}^{+0.23+0.05}$ & $2.41_{-0.24-0.06}^{+0.23+0.06}$ \\
\hline
\end{tabular}

TABLE XVII. Same as Table XVI for the LHC at $\sqrt{s}=8 \mathrm{TeV}$.

\begin{tabular}{|c|c|c|c|c|c|}
\hline$m_{H}$ & ABM11 & ABKM09 [16] & JR09 [20,21] & MSTW [22] & NN21 [23] \\
\hline 100 & $26.91_{-2.57-0.56}^{+2.72+0.56}$ & $26.82_{-2.59-0.75}^{+2.73+0.75}$ & $25.64_{-2.28-0.91}^{+2.44+0.91}$ & $28.86_{-2.85-0.44}^{+3.08+0.31}$ & $30.35_{-3.03-0.37}^{+3.36+0.37}$ \\
\hline 110 & $22.17_{-2.13-0.46}^{+2.21+0.46}$ & $22.05_{-2.13-0.61}^{+2.22+0.61}$ & $21.31_{-1.88-0.72}^{+2.01+0.72}$ & $23.84_{-2.36-0.37}^{+2.51+0.25}$ & $25.07_{-2.48-0.32}^{+2.74+0.32}$ \\
\hline 115 & $20.22_{-1.94-0.42}^{+2.02+0.42}$ & $20.10_{-1.94-0.56}^{+2.02+0.56}$ & $19.52_{-1.71-0.65}^{+1.83+0.65}$ & $21.78_{-2.15-0.33}^{+2.29+0.24}$ & $22.91_{-2.26-0.29}^{+2.49+0.29}$ \\
\hline 120 & $18.51_{-1.78-0.39}^{+1.84+0.39}$ & $18.39_{-1.78-0.51}^{+1.84+0.51}$ & $17.96_{-1.58-0.59}^{+1.68+0.59}$ & $19.97_{-1.98-0.31}^{+2.09+0.21}$ & $21.00_{-2.06-0.28}^{+2.28+0.28}$ \\
\hline 125 & $16.99_{-1.63-0.37}^{+1.69+0.37}$ & $16.87_{-1.63-0.47}^{+1.68+0.47}$ & $16.53_{-1.44-0.53}^{+1.54+0.53}$ & $18.36_{-1.82-0.28}^{+1.92+0.21}$ & $19.30_{-1.89-0.26}^{+2.09+0.26}$ \\
\hline 130 & $15.64_{-1.51-0.34}^{+1.55+0.34}$ & $15.52_{-1.50-0}^{+1.54+0}$ & $15.29_{-1.33-0.48}^{+1.42+0.48}$ & $16.94_{-1.68-0.27}^{+1.76+0.18}$ & $17.80_{-1.74-0.24}^{+1.92+0.24}$ \\
\hline 140 & $13.36_{-1.29-0.30}^{+1.31+0.30}$ & $13.25_{-1.29-0.38}^{+1.31+0.38}$ & $13.16_{-1.14+}^{+1.21+}$ & $14.52_{-1}^{+1}$ & $15.25_{-1.4}^{+1.6}$ \\
\hline 150 & $11.51_{-1.12-0.26}^{+1.13+0.26}$ & $11.42_{-1.11-0.34}^{+1.12+0.34}$ & $11.45_{-0.99-}^{+1.05+}$ & $12.56_{-1.2}^{+1.2}$ & $13.19_{-1.2}^{+1 .}$ \\
\hline 160 & $10.01_{-0.97-0.23}^{+0.98+0.23}$ & $9.92_{-0.97-0.29}^{+0.97+0.29}$ & $10.03_{-0.86-0.30}^{+0.91+0.30}$ & $10.96_{-1.09-0.18}^{+1.12+0.14}$ & $11.51_{-1.10-0.17}^{+1.22+0.17}$ \\
\hline 180 & $\begin{array}{l}7.74_{-0.76-0.19}^{+0.75+0.19}\end{array}$ & $7.67_{-0.75-0.24}^{+0.74+0.24}$ & $7.88_{-0.67-0.24}^{+0.71+0.24}$ & $8.55_{-0.85-0.14}^{+0.86+0.11}$ & $8.96_{-0.85-0.14}^{+0.94+0.14}$ \\
\hline 200 & $6.15_{-0.60-0.16}^{+0.59+0.16}$ & $6.10_{-0.60-0.20}^{+0.59+0.20}$ & $6.36_{-0.54-0.20}^{+0.56+0.20}$ & $6.84_{-0.68-0.12}^{+0.68+0.10}$ & $7.18_{-0.69-0.12}^{+0.73+0.12}$ \\
\hline 220 & $5.00_{-0.49-0.14}^{+0.48+0.14}$ & $4.96_{-0.48-0.17}^{+0.47+0.17}$ & $5.26_{-0.45-0.17}^{+0.46+0.17}$ & $5.61_{-0.56-0.10}^{+0.56+0.09}$ & $5.89_{-0.57-0.10}^{+0.59+0.10}$ \\
\hline 260 & $3.53_{-0.35-0.11}^{+0.34+0.11}$ & $3.52_{-0.35-0.13}^{+0.33+0.13}$ & $3.84_{-0.33-0.14}^{+0.32+0.14}$ & $4.04_{-0.41-0.08}^{+0.40+0.07}$ & $4.23_{-0.41-0.08}^{+0.41+0.08}$ \\
\hline 300 & $\begin{array}{r}2.72_{-0.27-0.09}^{+0.26+0.09} \\
\end{array}$ & $2.73_{-0.27-0.11}^{+0.26+0.11}$ & $3.06_{-0.27-0.13}^{+0.25+0.13}$ & $3.18_{-0.32-0.07}^{+0.31+0.07}$ & $3.33_{-0.33-0.07}^{+0.31+0.07}$ \\
\hline
\end{tabular}

significant at the level of $3-4 \sigma$. The reasons for the different gluon luminosities in the relevant $x$ range and the value of $\alpha_{s}\left(M_{Z}\right)$ have been illustrated in Sec. IV. The great sensitivity of the ggF rate to constraints from higher orders in QCD in the treatment of fixed-target DIS data has already been discussed extensively in Ref. [27].

Next in size comes the VBF channel. All numbers in Table XIX are computed with the VBFNNLO program
$[189,190]$ in the structure function approach, which describes VBF as a double DIS process, where two (virtual) vector-bosons $V_{i}$ (independently) emitted from the hadronic initial states fuse into a Higgs boson. Although the structure function approach to VBF is not truly exact at NNLO, it includes the bulk of the radiative corrections so that the remaining contributions are both parametrically small and kinematically suppressed; see Ref. [190]. The 
TABLE XVIII. Same as Table XVI for the LHC at $\sqrt{s}=14 \mathrm{TeV}$.

\begin{tabular}{|c|c|c|c|c|c|}
\hline$m_{H}$ & ABM11 & ABKM09 [16] & JR09 [20,21] & MSTW [22] & NN21 [23] \\
\hline 100 & $66.79_{-5.63-1.31}^{+6.13+1.31}$ & $67.29_{-5.78-1.80}^{+6.28+1.80}$ & $62.23_{-4.92-2.62}^{+5.46+2.62}$ & $70.76_{-6.23-1.12}^{+6.91+0.80}$ & $74.18_{-6.54-0.78}^{+7.50+0.78}$ \\
\hline 110 & $56.35_{-4.77-1.06}^{+5.11+1.06}$ & $56.62_{-4.88-1.47}^{+5.21+1.47}$ & $52.77_{-4.14-2.11}^{+4.58+2.11}$ & $59.75_{-5.27-0.95}^{+5.76+0.62}$ & $62.63_{-5.47-0.66}^{+6.26+0.66}$ \\
\hline 115 & $52.01_{-4.40-0.99}^{+4.70+0.99}$ & $52.20_{-4.49-1.35}^{+4.79+1.35}$ & $48.82_{-3.80-1.92}^{+4.21+1.92}$ & $55.17_{-4.85-0.84}^{+5.31+0.60}$ & $57.86_{-5.04-0.62}^{+5.74+0.62}$ \\
\hline 120 & $48.14_{-4.07-0.93}^{+4.35+0.93}$ & $48.26_{-4.14-1.25}^{+4.42+1.25}$ & $45.33_{-3.53-1.75}^{+3.89+1.75}$ & $51.12_{-4.50-0.80}^{+4.89+0.50}$ & $53.58_{-4.64-0.58}^{+5.31+0.58}$ \\
\hline 125 & $44.68_{-3.78-0.85}^{+4.02+0.85}$ & $44.75_{-3.85-1.16}^{+4.07+1.16}$ & $42.13_{-3.26-1.59}^{+3.60+1.59}$ & $47.47_{-4.18-0.71}^{+4.52+0.50}$ & $49.77_{-4.30-0.54}^{+4.91+0.54}$ \\
\hline 130 & $41.59_{-3.53-0.80}^{+3.72+0.80}$ & $41.61_{-3.58-1.07}^{+3.77+1.07}$ & $39.32_{-3.02-1.45}^{+3.35+1.45}$ & $44.22_{-3.91-0.70}^{+4.18+0.42}$ & $46.35_{-3.99-0.51}^{+4.55+0.51}$ \\
\hline 140 & $36.28_{-3.09-0.70}^{+3.23+0.70}$ & $36.24_{-3.12-0.94}^{+3.26+0.94}$ & $34.46_{-2.65-1.22}^{+2.90+1.22}$ & $38.63_{-3.41-0.59}^{+3.63+0.37}$ & $40.49_{-3.47-0.45}^{+3.94+0.45}$ \\
\hline 150 & $31.92_{-2.72-0.61}^{+2.82+0.61}$ & $31.85_{-2.74-0.82}^{+2.85+0.82}$ & $30.49_{-2.32-1.04}^{+2.56+1.04}$ & $34.05_{-3.01-0.51}^{+3.18+0.33}$ & $35.69_{-3.04-0.41}^{+3.45+0.41}$ \\
\hline 160 & $28.32_{-2.42-0.54}^{+2.49+0.54}$ & $28.22_{-2.44-0.72}^{+2.50+0.72}$ & $27.16_{-2.06-0.90}^{+2.25+0.90}$ & $30.25_{-2.67-0.44}^{+2.80+0.30}$ & $31.69_{-2.67-0.37}^{+3.04+0.37}$ \\
\hline 180 & $22.75_{-1.96-0.44}^{+1.98+0.44}$ & $22.62_{-1.95-0.59}^{+1.99+0.59}$ & $22.03_{-1.65-0.69}^{+1.80+0.69}$ & $24.39_{-2.16-0.36}^{+2.23+0.24}$ & $25.56_{-2.15-0.31}^{+2.41+0.31}$ \\
\hline 200 & $18.74_{-1.61-0.37}^{+1.62+0.37}$ & $18.61_{-1.61-0.49}^{+1.62+0.49}$ & $18.33_{-1.38-0.54}^{+1.47+0.54}$ & $20.17_{-1.79-0.29}^{+1.83+0.21}$ & $21.16_{-1.79-0.27}^{+1.94+0.27}$ \\
\hline 220 & $15.78_{-1.36-0.32}^{+1.36+0.32}$ & $15.66_{-1.35-0.42}^{+1.35+0.42}$ & $15.61_{-1.17-0.45}^{+1.23+0.45}$ & $17.05_{-1.51-0.25}^{+1.53+0.18}$ & $17.90_{-1.52-0.24}^{+1.61+0.24}$ \\
\hline 260 & $11.91_{-1.04-0.26}^{+1.01+0.26}$ & $11.81_{-1.03-0.33}^{+1.00+0.33}$ & $12.03_{-0.91-0.34}^{+0.91+0.34}$ & $12.98_{-1.15-0.20}^{+1.15+0.16}$ & $13.64_{-1.16-0.19}^{+1.18+0.19}$ \\
\hline 300 & $9.80_{-0.86-0.23}^{+0.83+0.23}$ & $9.73_{-0.85-0.28}^{+0.82+0.28}$ & $10.11_{-0.77-0.29}^{+0.75+0.29}$ & $10.79_{-0.96-0.17}^{+0.95+0.14}$ & $11.34_{-0.97-0.17}^{+0.95+0.17}$ \\
\hline
\end{tabular}

TABLE XIX. Total VBF cross sections at the $\mathrm{LHC}(\sqrt{s}=7 \mathrm{TeV})$ for different PDF sets and to NNLO accuracy as computed with VBFNNLO $[189,190]$. Errors shown are the scale uncertainties evaluated by varying $\mu_{r}$ and $\mu_{f}$ in the interval $\mu_{r}, \mu_{f} \in[Q / 4,4 Q]$ and, respectively, the PDF uncertainties. Numbers are in pb.

\begin{tabular}{|c|c|c|c|c|c|}
\hline$m_{H}$ & ABM11 & ABKM09 [16] & JR09 [20,21] & MSTW [22] & NN21 [23] \\
\hline 100 & $1.673_{-0.022-0.020}^{+0.024+0.020}$ & $1.643_{-0.026-0.012}^{+0.022+0.012}$ & $1.599_{-0.017-0.022}^{+0.023+0.022}$ & $1.616_{-0.034-0.029}^{+0.025+0.029}$ & $1.603_{-0.028-0.021}^{+0.028+0.021}$ \\
\hline 110 & $1.513_{-0.019-0.018}^{+0.025+0.018}$ & $1.483_{-0.022-0.011}^{+0.026+0.011}$ & $1.453_{-0.020-0.021}^{+0.014+0.021}$ & $1.460_{-0.027-0.026}^{+0.025+0.026}$ & $1.448_{-0.023-0.019}^{+0.027+0.019}$ \\
\hline 115 & $1.440_{-0.021-0.017}^{+0.021+0.017}$ & $1.411_{-0.014-0.011}^{+0.022+0.011}$ & $1.388_{-0.022-0.020}^{+0.016+0.020}$ & $1.391_{-0.028-0.025}^{+0.026+0.025}$ & $1.378_{-0.024-0.018}^{+0.029+0.018}$ \\
\hline 120 & $1.373_{-0.018-0.016}^{+0.022+0.016}$ & $1.345_{-0.016-0.010}^{+0.022+0.010}$ & $1.321_{-0.018-0.019}^{+0.018+0.019}$ & $1.324_{-0.023-0.024}^{+0.025+0.024}$ & $1.318_{-0.028-0.017}^{+0.021+0.017}$ \\
\hline 125 & $1.307_{-0.018-0.016}^{+0.023+0.016}$ & $1.285_{-0.022-0.010}^{+0.020+0.010}$ & $1.260_{-0.020-0.019}^{+0.017+0.019}$ & $1.264_{-0.023-0.023}^{+0.023+0.023}$ & $1.252_{-0.019-0.016}^{+0.026+0.016}$ \\
\hline 130 & $1.244_{-0.014-0.015}^{+0.025+0.015}$ & $1.223_{-0.013-0.009}^{+0.022+0.009}$ & $1.203_{-0.017-0.018}^{+0.017+0.018}$ & $1.203_{-0.021-0.022}^{+0.026+0.022}$ & $1.195_{-0.018-0.016}^{+0.027+0.016}$ \\
\hline 140 & $1.137_{-0.015-0.014}^{+0.016+0.014}$ & $1.116_{-0.013-0.008}^{+0.021+0.008}$ & $1.098_{-0.016-0.017}^{+0.018+0.017}$ & $1.099_{-0.019-0.020}^{+0.024+0.020}$ & $1.093_{-0.020-0.015}^{+0.021+0.015}$ \\
\hline 150 & $1.038_{-0.013-0.013}^{+0.019+0.013}$ & $1.018_{-0.011-0.008}^{+0.021+0.008}$ & $1.006_{-0.014-0.016}^{+0.015+0.016}$ & $1.003_{-0.016-0.019}^{+0.021+0.019}$ & $0.999_{-0.019-0.013}^{+0.020+0.013}$ \\
\hline 160 & $0.950_{-0.011-0.012}^{+0.018+0.012}$ & $0.934_{-0.012-0.007}^{+0.017+0.007}$ & $0.923_{-0.014-0.015}^{+0.016+0.015}$ & $0.918_{-0.013-0.017}^{+0.018+0.017}$ & $0.915_{-0.014-0.012}^{+0.017+0.012}$ \\
\hline 180 & $0.800_{-0.009-0.010}^{+0.016+0.010}$ & $0.785_{-0.007-0.006}^{+0.016+0.006}$ & $0.781_{-0.010-0.014}^{+0.013+0.014}$ & $0.773_{-0.011-0.015}^{+0.019+0.015}$ & $0.771_{-0.011-0.011}^{+0.018+0.011}$ \\
\hline 200 & $0.679_{-0.008-0.009}^{+0.013+0.009}$ & $0.669_{-0.007-0.005}^{+0.012+0.005}$ & $0.665_{-0.008-0.012}^{+0.011+0.012}$ & $0.658_{-0.010-0.013}^{+0.014+0.013}$ & $0.656_{-0.009-0.009}^{+0.016+0.009}$ \\
\hline 220 & $0.580_{-0.006-0.008}^{+0.011+0.008}$ & $0.571_{-0.005-0.005}^{+0.012+0.005}$ & $0.570_{-0.006-0.011}^{+0.010+0.011}$ & $0.562_{-0.007-0.011}^{+0.013+0.011}$ & $0.561_{-0.007-0.008}^{+0.012+0.008}$ \\
\hline 260 & $0.429_{-0.003-0.006}^{+0.010+0.006}$ & $0.424_{-0.003-0.004}^{+0.009+0.004}$ & $0.425_{-0.004-0.009}^{+0.008+0.009}$ & $0.417_{-0.005-0.008}^{+0.010+0.008}$ & $0.418_{-0.005-0.006}^{+0.010+0.006}$ \\
\hline 300 & $0.324_{-0.002-0.004}^{+0.008+0.004}$ & $0.321_{-0.003-0.003}^{+0.007+0.003}$ & $0.323_{-0.003-0.007}^{+0.006+0.007}$ & $0.316_{-0.003-0.007}^{+0.008+0.007}$ & $0.316_{-0.003-0.005}^{+0.007+0.005}$ \\
\hline
\end{tabular}

residual theory uncertainty based on the scale variation is rather small and the cross sections for all PDF sets considered in Table XIX agree well, typically within $1-2 \sigma$ of the PDF uncertainties.

Last in line we consider the Higgs-strahlung process, that is the associated $W H$ and $Z H$ production using the NNLO QCD corrections of Ref. [191]. See also Ref. [201] for fully exclusive QCD calculations at NNLO. The dominant part of the hard partonic cross section is the same as for $W$ and $Z$ boson production discussed above in Sec. VA, so that essentially the same PDFs are probed, although at slightly larger values of $x$. The numbers in Tables XX and XXI do not contain the gluon induced contribution for $\sigma(Z H)$ [202]. Top-quark mediated effects, which we neglect here, yield small perturbative corrections that are largely independent of the production model, that is to say of the parton luminosity; see also Ref. [203] for a recent discussion. For the Higgs-strahlung the scale 
TABLE XX. Total cross sections $\sigma(W H)$ for associated Higgs production in the $W H$ mode at the LHC $(\sqrt{s}=7 \mathrm{TeV})$ for different PDF sets and to NNLO accuracy. Errors shown are the scale uncertainty based on the shifts $\mu=\left(m_{H}+M_{W}\right) / 2$ and $\mu=2\left(m_{H}+M_{W}\right)$ and, respectively, the $1 \sigma$ PDF uncertainty. Numbers are in fb.

\begin{tabular}{lccccc}
\hline \hline$m_{H}$ & ABM11 & ABKM09 [16] & JR09 [20,21] & MSTW [22] & NN21 [23] \\
\hline 100 & $1298_{-37-17}^{+14+17}$ & $1270_{-37-14}^{+14+14}$ & $1229_{-35-17}^{+17+17}$ & $1256_{-36-18}^{+14+22}$ & $1273_{-40-20}^{+19+20}$ \\
110 & $960_{-27-13}^{+10+13}$ & $938_{-27-10}^{+10+10}$ & $911_{-26-12}^{+12+12}$ & $930_{-27-13}^{+10+16}$ & $942_{-30-15}^{+14+15}$ \\
115 & $832_{-24-11}^{+9+11}$ & $812_{-23-9}^{+9+9}$ & $790_{-23-10}^{+11+10}$ & $805_{-23-12}^{+8+14}$ & $816_{-26-13}^{+12+13}$ \\
120 & $723_{-20-10}^{+7+10}$ & $706_{-20-8}^{+7+8}$ & $687_{-20-9}^{+9+9}$ & $700_{-20-10}^{+7+12}$ & $709_{-22-11}^{+10+11}$ \\
125 & $631_{-18-9}^{+6+9}$ & $616_{-18-7}^{+6+7}$ & $600_{-17-8}^{+8+8}$ & $611_{-18-9}^{+6+11}$ & $619_{-19-10}^{+9+10}$ \\
130 & $553_{-16-8}^{+5+8}$ & $539_{-15-6}^{+5+6}$ & $527_{-16-5}^{+6+5}$ & $536_{-16-8}^{+5+9}$ & $543_{-18-9}^{+7+9}$ \\
140 & $428_{-12-6}^{+5+6}$ & $417_{-11-5}^{+5+5}$ & $409_{-12-4}^{+5+4}$ & $415_{-12-6}^{+4+7}$ & $421_{-14-7}^{+6+7}$ \\
150 & $336_{-9-5}^{+3+5}$ & $327_{-9-4}^{+3+4}$ & $321_{-9-3}^{+5+3}$ & $326_{-9-5}^{+3+6}$ & $330_{-10-6}^{+5+6}$ \\
160 & $267_{-8-4}^{+2+4}$ & $259_{-7-3}^{+3+3}$ & $256_{-8-2}^{+3+2}$ & $259_{-8-4}^{+2+5}$ & $262_{-8-5}^{+4+5}$ \\
\hline \hline
\end{tabular}

uncertainty is typically small and the predictions of the various PDF sets in Tables XX and XXI agree well within the quoted PDF uncertainties.

\section{Top-quark pair production}

Here we present results for top-quark pair production at the LHC at $\sqrt{s}=7 \mathrm{TeV}$. The cross section is driven predominantly by the gluon luminosity and the value of $\alpha_{s}$, much like in the case of Higgs production in ggF. By far the largest parametric dependence of the cross section resides, however, in the value of the top-quark mass. This is currently quoted with an experimental uncertainty of less than $1 \%$ as $m_{t}=172.9 \pm 1.1 \mathrm{GeV}$ based on the kinematic reconstruction from the decay products and comparison to Monte Carlo simulations [98]. This value is commonly considered to be the pole mass of the top-quark, although one should keep in mind, that the reconstruction of the

TABLE XXI. Same as Table XX for $\sigma(Z H)$ and the scale uncertainties based on the shifts $\mu=\left(m_{H}+M_{Z}\right) / 2$ and $\mu=2\left(m_{H}+M_{Z}\right)$.

\begin{tabular}{cccccc}
\hline \hline$m_{H}$ & ABM11 & $\begin{array}{c}\text { ABKM09 } \\
{[16]}\end{array}$ & $\begin{array}{c}\text { JR09 } \\
{[20,21]}\end{array}$ & $\begin{array}{c}\text { MSTW } \\
{[22]}\end{array}$ & $\begin{array}{c}\text { NN21 } \\
{[23]}\end{array}$ \\
\hline 100 & $669_{-20-9}^{+7+9}$ & $656_{-20-7}^{+7+7}$ & $635_{-19-5}^{+9+5}$ & $651_{-19-9}^{+7+10}$ & $655_{-22-10}^{+10+10}$ \\
110 & $499_{-15-7}^{+5+7}$ & $488_{-14-5}^{+6+5}$ & $474_{-14-4}^{+7+4}$ & $486_{-15-7}^{+5+9}$ & $488_{-16-8}^{+7+8}$ \\
115 & $433_{-13-6}^{+5+6}$ & $424_{-12-5}^{+5+5}$ & $413_{-13-4}^{+5+4}$ & $422_{-13-6}^{+4++}$ & $424_{-14-7}^{+6+7}$ \\
120 & $378_{-11-5}^{+4+5}$ & $370_{-11-4}^{+4+4}$ & $360_{-11-3}^{+5+3}$ & $368_{-11-5}^{+4+7}$ & $370_{-12-6}^{+5+6}$ \\
125 & $331_{-10-5}^{+3+5}$ & $323_{-9-4}^{+4+4}$ & $316_{-10-3}^{+4+3}$ & $322_{-9-5}^{+4+6}$ & $324_{-11-5}^{+5+5}$ \\
130 & $290_{-8-4}^{+3+4}$ & $284_{-8-3}^{+3+3}$ & $277_{-8-2}^{+4+2}$ & $283_{-8-4}^{+3+5}$ & $285_{-10-5}^{+4+5}$ \\
140 & $226_{-7-3}^{+2+3}$ & $221_{-7-2}^{+2+2}$ & $216_{-6-2}^{+3+2}$ & $221_{-7-3}^{+2+4}$ & $222_{-8-4}^{+3+4}$ \\
150 & $178_{-5-3}^{+2+3}$ & $174_{-5-2}^{+2+2}$ & $171_{-5-2}^{+2+2}$ & $174_{-5-3}^{+2+3}$ & $175_{-6-3}^{+2+3}$ \\
160 & $142_{-4-2}^{+1+2}$ & $138_{-4-2}^{+2+2}$ & $136_{-4-1}^{+2+1}$ & $139_{-5-2}^{+1+3}$ & $139_{-4-2}^{+2+2}$ \\
\hline \hline
\end{tabular}

top-quark momenta from the observed (color-neutral) hadron momenta carries a further intrinsic uncertainty of $\mathcal{O}\left(\Lambda_{\mathrm{QCD}}\right)$. Top-quark mass measurements in a well-defined scheme for $m_{t}$ from the inclusive cross section at the Tevatron give lower masses, with a larger uncertainty though [192,204].

In Table XXII we summarize the cross section values for a range of top-quark masses $165 \leq m_{t} \leq 180$ in the pole mass scheme. The theoretical uncertainty at (approximate) NNLO is quantified by a variation of scale in the range $m_{t} / 2 \leq \mu \leq 2 m_{t}$. We see that the predictions of ABKM09 and ABM11 are largely the same, the latter being slightly smaller due to a smaller gluon PDF in the relevant $x$ range; cf. Fig. 13. In comparison to other PDFs, the predictions based on ABM11 in Table XXII are significantly smaller, and it seems that precision measurements of the cross section at the LHC can potentially constrain the gluon PDF. This discriminating power, however, relies critically on the accurate knowledge of all other nonperturbative parameters, in particular $m_{t}$. See Tables XXIII and XXIV for the LHC at 8 and $14 \mathrm{TeV}$, respectively.

Cross section predictions using the running top-quark mass in the $\overline{\mathrm{MS}}$ scheme instead of the pole mass definition for $m_{t}$ in Table XXII generally display improved stability of the perturbative expansion and good properties of apparent convergence, which is reflected in smaller uncertainties due to the scale variation; see e.g., Ref. [192]. The PDF uncertainties given in Table XXII and the differences between the various PDF sets are, of course, largely unaffected.

\section{Hadronic jet production}

The hadronic jet production is sensitive to all nucleon PDFs, in particular, to the large- $x$ gluon distribution, which is not sufficiently sensitive to other processes employed in PDF fits. Therefore, the inclusive jet production data obtained by the Tevatron collider experiments $[108,110]$ are used in the PDF fits of Refs. [22,24,100] in order to provide 
TABLE XXII. Total cross section for top-quark pair production at the $\mathrm{LHC}(\sqrt{s}=7 \mathrm{TeV})$ for different PDF sets and to (approximate) NNLO accuracy as computed with HATHOR (version 1.2) [193] as a function of the pole mass $m_{t}$. Errors shown are the scale uncertainty based on the shifts $\mu=m_{t} / 2$ and $\mu=2 m_{t}$ and, respectively, the $1 \sigma$ PDF uncertainty. All rates are in pb.

\begin{tabular}{|c|c|c|c|c|c|}
\hline$m_{t}$ & ABM11 & ABKM09 [16] & JR09 [20,21] & MSTW [22] & NN21 [23] \\
\hline 165 & $167.9_{-9.3-7.5}^{+3.6+7.5}$ & $171.4_{-9.3-9.6}^{+3.5+9.6}$ & $204.3_{-9.1-14.7}^{+3.1+14.7}$ & $209.9_{-11.8-5.6}^{+4.0+5.5}$ & $216.1_{-10.8-5.8}^{+4.6+5.8}$ \\
\hline 166 & $162.6_{-9.0-7.3}^{+3.5+7.3}$ & $166.1_{-9.0-9.3}^{+3.4+9.3}$ & $197.2_{-7.9-14.4}^{+3.9+14.4}$ & $203.5_{-11.4-5.4}^{+3.9+5.3}$ & $209.4_{-10.5-5.7}^{+4.4+5.7}$ \\
\hline 167 & $157.5_{-8.8-7.0}^{+3.4+7.0}$ & $160.9_{-8.7-9.0}^{+3.3+9.0}$ & $191.2_{-7.6-14.0}^{+3.8+14.0}$ & $197.3_{-11.1-5.3}^{+3.8+5.2}$ & $202.9_{-10.1-5.5}^{+4.3+5.5}$ \\
\hline 168 & $152.5_{-8.5-6.8}^{+3.3+6.8}$ & $155.9_{-8.5-8.8}^{+3.2+8.8}$ & $185.5_{-7.4-13.7}^{+3.7+13.7}$ & $191.3_{-10.8-5.1}^{+3.7+5.1}$ & $196.7_{-9.8-5.4}^{+4.2+5.4}$ \\
\hline 169 & $147.8_{-8.2-6.7}^{+3.2+6.7}$ & $151.1_{-8.2-8.5}^{+3.1+8.5}$ & $179.8_{-7.1-13.3}^{+3.7+13.3}$ & $185.5_{-10.4-5.0}^{+3.7+4.9}$ & $190.6_{-9.4-5.2}^{+4.2+5.2}$ \\
\hline 170 & $143.2_{-8.0-6.5}^{+3.2+6.5}$ & $146.4_{-8.0-8.3}^{+3.0+8.3}$ & $174.5_{-6.9-13.0}^{+3.6+13.0}$ & $179.9_{-10.1-4.9}^{+3.5+4.8}$ & $184.9_{-9.1-5.1}^{+4.1+5.1}$ \\
\hline 171 & $138.8_{-7.7-6.3}^{+3.1+6.3}$ & $141.9_{-7.7-8.1}^{+3.0+8.1}$ & $169.2_{-6.6-12.7}^{+3.6+12.7}$ & $174.5_{-9.7-4.7}^{+3.5+4.6}$ & $179.2_{-8.7-4.9}^{+4.0+4.9}$ \\
\hline 172 & $134.5_{-7.5-6.1}^{+3.0+6.1}$ & $137.6_{-7.5-7.9}^{+2.9+7.9}$ & $164.2_{-6.5-12.4}^{+3.5+12.4}$ & $169.3_{-9.5-4.6}^{+3.3+4.5}$ & $173.9_{-8.5-4.8}^{+3.9+4.8}$ \\
\hline 173 & $130.4_{-7.2-5.9}^{+2.9+5.9}$ & $133.4_{-7.3-7.6}^{+2.8+7.6}$ & $159.3_{-6.3-12.0}^{+3.4+12.0}$ & $164.3_{-9.2-4.5}^{+3.3+4.4}$ & $168.7_{-8.2-4.7}^{+3.8+4.7}$ \\
\hline 174 & $126.4_{-7.0-5.8}^{+2.8+5.8}$ & $129.4_{-7.1-7.4}^{+2.7+7.4}$ & $154.6_{-6.0-11.8}^{+3.4+11.8}$ & $\begin{array}{r}159.4_{-8.9-4.4}^{+3.2+4.3}\end{array}$ & $163.7_{-7.9-4.6}^{+3.8+4.6}$ \\
\hline 175 & $122.6_{-6.8-5.6}^{+2.8+5.6}$ & $125.5_{-6.8-7.2}^{+2.7+7.2}$ & $150.0_{-5.8-11.5}^{+3.3+11.5}$ & $154.7_{-8.7-4.2}^{+3.1+4.2}$ & $158.9_{-7.8-4.4}^{+3.6+4.4}$ \\
\hline 176 & $118.9_{-6.6-5.5}^{+2.7+5.5}$ & $121.8_{-6.6-7.0}^{+2.6+7.0}$ & $145.6_{-5.6-11.3}^{+3.3+11.3}$ & $150.2_{-8.4-4.1}^{+3.1+4.1}$ & $154.2_{-7.5-4.3}^{+3.5+4.3}$ \\
\hline 177 & $115.3_{-6.4-5.3}^{+2.6+5.3}$ & $\begin{array}{r}118.1_{-6.5-6.9}^{+2.5+6.9} \\
\end{array}$ & $141.3_{-5.5-11.0}^{+3.1+11.0}$ & $\begin{array}{l}145.8_{-8.2-4.0}^{+2.9+4.0} \\
\end{array}$ & $\begin{array}{l}149.8_{-7.4-4.2}^{+3.3+4.2} \\
\end{array}$ \\
\hline 178 & $111.9_{-6.2-5.2}^{+2.5+5.2}$ & $114.6_{-6.3-6.7}^{+2.5+6.7}$ & $137.2_{-5.2-10.7}^{+3.1+10.7}$ & $141.6_{-7.9-3.9}^{+2.9+3.9}$ & $145.4_{-7.1-4.1}^{+3.3+4.1}$ \\
\hline 179 & $108.5_{-6.0-5.0}^{+2.5+5.0}$ & $111.3_{-6.1-6.5}^{+2.4+6.5}$ & $133.4_{-5.2-10.4}^{+2.8+10.4}$ & $\begin{array}{r}137.6_{-7.8-3.8}^{+2.7+3.8}\end{array}$ & $141.2_{-7.0-4.0}^{+3.1+4.0}$ \\
\hline 180 & $105.3_{-5.9-4.9}^{+2.4+4.9}$ & $108.0_{-5.9-6.3}^{+2.3+6.3}$ & $129.6_{-5.0-10.2}^{+2.8+10.2}$ & $133.6_{-7.6-3.7}^{+2.7+3.7}$ & $137.1_{-6.8-3.9}^{+3.0+3.9}$ \\
\hline
\end{tabular}

better constraints on the large- $x$ gluon. The calculation of the full NNLO QCD corrections to this process is still in progress (see Refs. [205,206] and references therein). This precludes a consistent use of the Tevatron jet data in our NNLO PDF fit. Nevertheless, in order to check any potential impact of the jet Tevatron data on our PDFs, we have performed trial variants of the NNLO ABKM09 fit with the Tevatron jet data added [102]. The NLO QCD corrections $[207,208]$ and the partial (soft gluon enhanced) NNLO corrections due to threshold resummation [112] have been computed with the FastNLO tool $[209,210]$.

In order to allow more flexibility of the large- $x$ gluon distribution we have added the term $\gamma_{2, g} x^{2}$ to the polynomial of Eq. (4.9) with an additional fitted parameter $\gamma_{2, g}$ making sure that further expansions of this polynomial do not improve the quality of those fits. In general, the Tevatron jet data overshoot the ABKM09 predictions; nevertheless, they can be smoothly accommodated in the fit. The typical value of $\chi^{2} / N D P \approx 1$ is achieved with account of the error correlations for the jet data sets of Refs. [108-111] once they are included into the NNLO ABKM09 fit. Meanwhile the various data sets demonstrate a somewhat different trend with respect to the ABKM09 predictions. For example, the offset of the D0 inclusive jet data [110] does not depend on the jet energy $E_{T}$ and therefore may be attributed to the impact of the currently missing full NNLO corrections; cf. Figs. 26 and 27. In contrast, for the CDF data of Ref. [108] obtained with the
$k_{T}$ jet algorithm the pulls rise with $E_{T}$ and can be reduced only by means of a modification of the PDF shapes.

The values of $\alpha_{s}$ extracted from the trial ABKM09 fits with the Tevatron jet data included are compared with the nominal ABKM09 value in Table VII. At most, they are bigger by $1 \sigma$, while for the $\mathrm{CDF}$ cone jet algorithm data [109] the central value of $\alpha_{s}$ is even the same. A recent evaluation of $\alpha_{s}$ using the ATLAS inclusive jet cross section data yields $\alpha_{s}\left(M_{Z}\right)=0.1151$ at NLO [211]. The predictions for the light Higgs production cross section, which are defined by the gluon distribution at $x \leqq 0.1$, are also not very sensitive to the constraints coming from the Tevatron data; cf. Table XXV. The impact of the Tevatron jet data on the large- $x$ gluon distribution is more significant. However, in this context we note that the Tevatron dijet and 3-jet production data are in good agreement with the ABKM09 predictions [212], in contrast to the case of inclusive jet production at Tevatron. The analysis of Tevatron data 3-jet production has also shown that the predictions of MSTW [22] agree even better with the data of Ref. [213] than ABKM09. However, for the case of CT10 [24] this is opposite.

The trend of the first LHC data on the jet production with respect to the various PDF predictions is different from the Tevatron measurements. The ABKM09 predictions are in better agreement with the CMS and ATLAS inclusive data of Refs. $[115,214]$ than the predictions based on the PDFs of Refs. [22,24,100], which were tuned to the Tevatron 
Do(1jet)

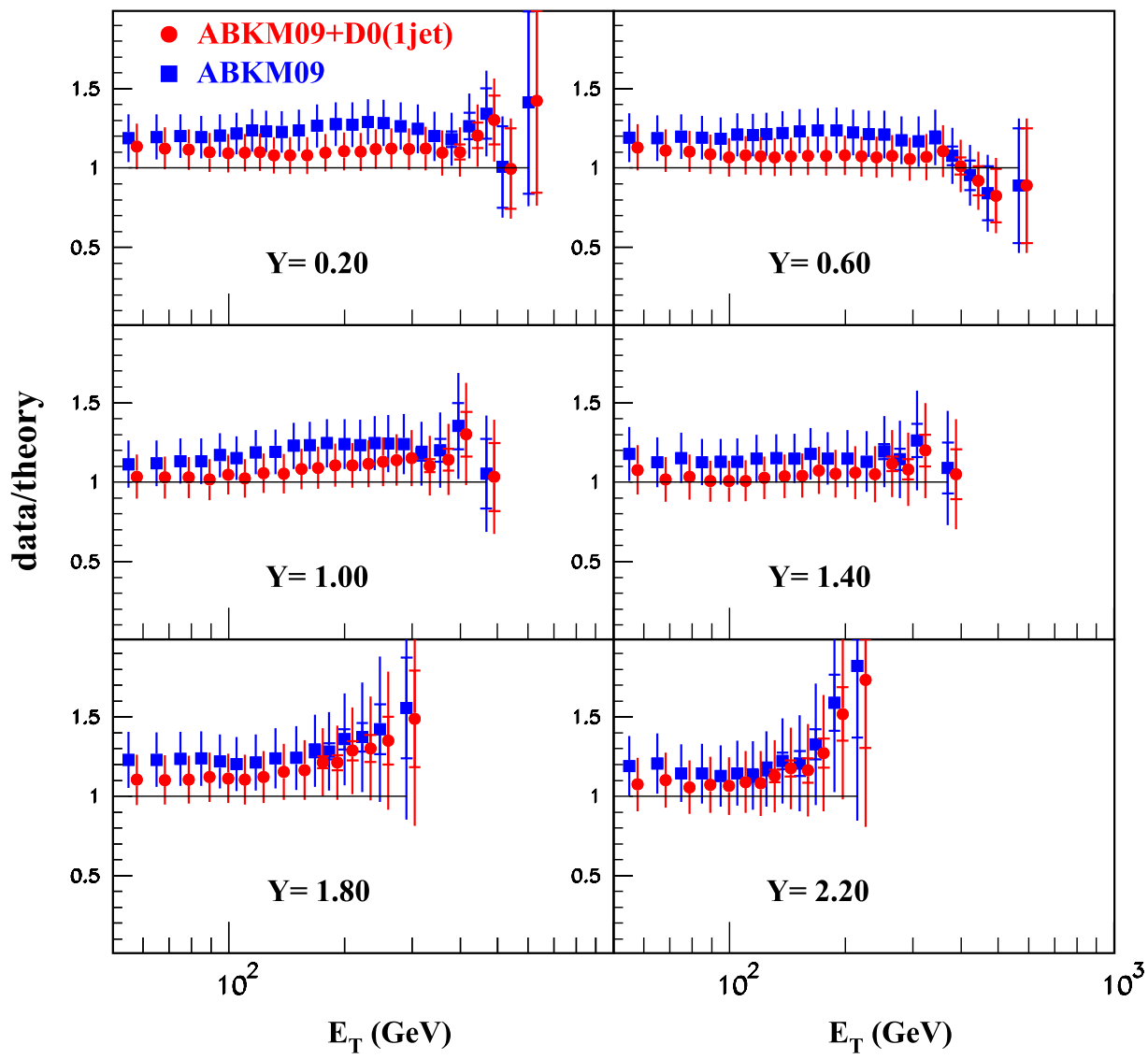

FIG. 26 (color online). Cross section data for 1-jet inclusive production from the D0 Collaboration [110] as a function of the jet's transverse energy $E_{T}$ for the renormalization and factorization scales equal to $E_{T}$ compared to the result of Ref. [16] (circles) and a refit including this data (squares) including the NNLO threshold resummation corrections to the jet production [112].

inclusive jet data. In Figs. 28 and 29 we show a comparison of the result of the present fit and of MSTW [22] with LHC jet data from ATLAS [214] and from CMS [215] calculated with the updated version of the FastNLO code [210]. The trend of these data in comparison to the predictions is the same for both experiments. We find good agreement at large transverse energy $E_{T}$ and small rapidity $Y$. The predictions overshoot the data for $E_{T}<100 \mathrm{GeV}$, i.e., in a range where the theory description is evidently incomplete. The predictions also overshoot the data (within the errors) at large $Y$. In summary this is the opposite trend as compared to the Tevatron data. If included in the PDF analysis and taking only data with $E_{T}>100-150 \mathrm{GeV}$ say, the LHC jet data may potentially lead to a decrease of the large- $x$ gluons. That said, it should be kept in mind that jet data from the LHC is still subject to large systematic errors, though. In summary, these ambiguities in the data as well as the limitations in the current theoretical treatment prevent the use of hadronic jet data in our fit.

Finally let us also comment on Ref. [28] in this context, which has studied the compatibility of the Tevatron jet data with the predictions based on the PDFs of ABKM09 [16] (see also Ref. [102]). The particular focus of Ref. [28] has been on shifts in the normalization uncertainty of the experimental data. Unfortunately, the study of Ref. [28] overestimates the statistical significance of such shifts in the normalization errors, since the PDF errors in the ABKM09 PDF sets have not been taken into account. The latter, however, should have a significant impact on the comparison because the large- $x$ gluon PDF of ABKM09, which is most relevant for such comparisons to Tevatron jet data, carries quite a large uncertainty in itself. This is corroborated by the fact that the value of $\chi^{2}$ obtained in the variants of the ABKM09 fit with the Tevatron data included [102] are quite good, illustrating in an indirect manner the irrelevance of Ref. [28].

\section{E. LHAPDF library}

For the cross section computations presented here we have used the LHAPDF library [216,217] to interface to our PDFs and to those of other groups. For wider public use and to facilitate cross section computations by the interested reader, we provide the results of the current analysis 


\section{CDF(1jet)}

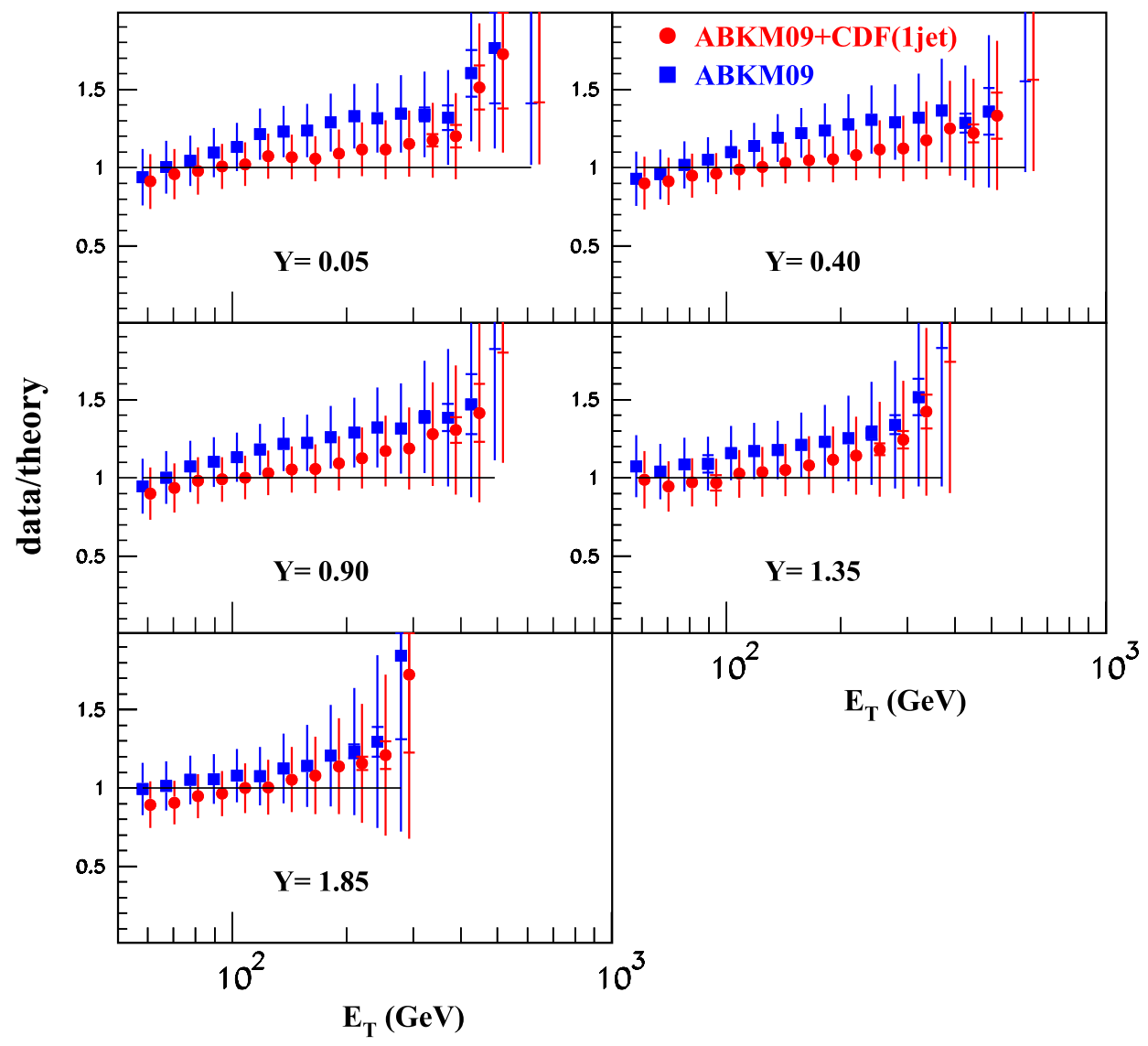

FIG. 27 (color online). Same as Fig. 26 for the cross section data for 1-jet inclusive production from the CDF Collaboration using a $k_{T}$ jet algorithm [108].

in the form of data grids accessible with the most recent version lhapdf-5.8.7 of the LHAPDF library, which can be obtained from http://projects.hepforge.org/lhapdf.

In detail, we provide three NLO grids for $n_{f}=3,4,5$ flavors,

$$
\begin{aligned}
& \text { abm11_3n_nlo.LHgrid }(0+28), \\
& \text { abm11_4n_nlo.LHgrid }(0+28), \\
& \text { abm11_5n_nlo.LHgrid }(0+28),
\end{aligned}
$$

with the central fit and 28 additional sets for the combined symmetric uncertainty on the PDFs and on $\alpha_{s}$ from Eqs. (4.12) and (4.13), the heavy-quark masses from Eq. (4.15), and the deuteron correction; cf. Fig. 1. Likewise at NNLO, we have

$$
\begin{aligned}
& \text { abm11_3n_nnlo.LHgrid }(0+28), \\
& \text { abm11_4n_nnlo.LHgrid }(0+28), \\
& \text { abm11_5n_nnlo.LHgrid }(0+28) .
\end{aligned}
$$

For future measurements of the strong coupling constant, e.g., from data on hadronic jet production as well as for detailed phenomenological studies of the parametric dependence of observables on $\alpha_{s}$, we also provide grids with fixed values of $\alpha_{s}$; see the discussion in Sec. IV B. At NLO these are 20 sets in the range $\alpha_{s}=0.11 \ldots 0.13$ and at NNLO 16 sets for $\alpha_{s}=0.105 \ldots 0.12$; cf. also Fig. 17. The sets for the $\alpha_{s}$ scan are denoted

$$
\begin{aligned}
& \text { abm11_5n_as_nlo.LHgrid }(0+20) \text {, } \\
& \text { abm11_5n_as_nnlo.LHgrid }(0+16) \text {. }
\end{aligned}
$$

During the computation of the cross sections with the LHAPDF library, we noticed significant differences in the run time of our programs, when determining the cross section value and its uncertainty for a particular PDF set. Usually, this requires sampling of the error PDFs that describe the error of the corresponding parameter in a given set. The run time, of course, depends on the number of parameters in a given set, but also on the parametrization of the PDF grid and details of the lookup algorithm. Efficiency for phenomenological studies requires sufficiently precise and at same time fast cross section computations. 
NNLO(appox.) $\mu_{\mathbf{R}}=\mu_{\mathbf{F}}=\mathbf{E}_{\mathbf{T}}$

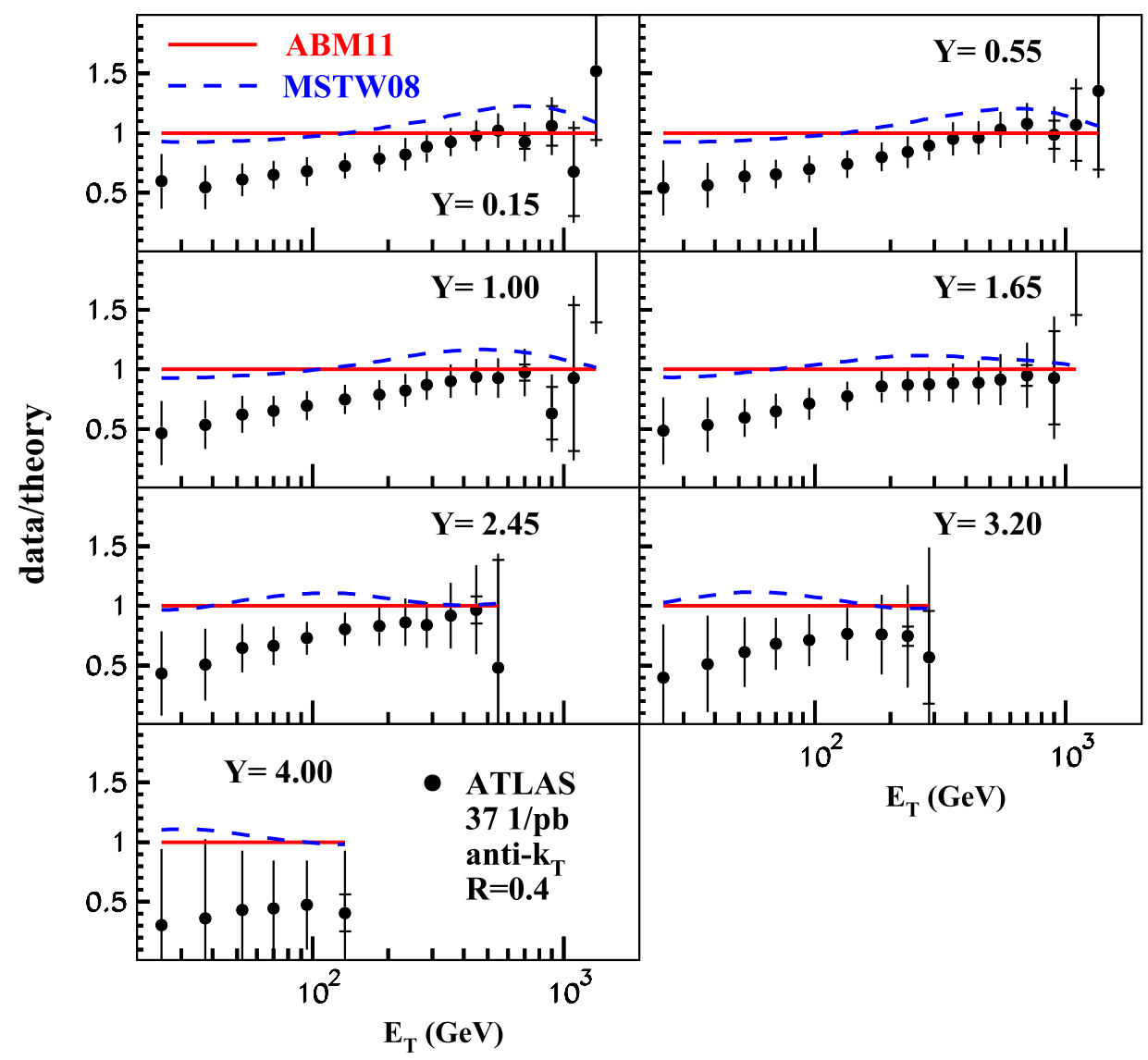

FIG. 28 (color online). Cross section data for 1-jet inclusive production from the ATLAS Collaboration [110] as a function of the jet's transverse energy $E_{T}$ for $\mu_{R}=\mu_{F}=E_{T}$ compared to the result of the present analysis (solid lines) and to MSTW [22] (dashed lines). The theory predictions include the NNLO threshold resummation corrections to the jet production [112].

Interestingly, for all observables discussed above, we have found the same pattern for the required run times. The grids of ABKM09 [16] and ABM11 are both equally fast, i.e., $t_{\mathrm{ABKM} 09} \simeq t_{\mathrm{ABM} 11}$. Next come the JR09 [20,21] grids that are slightly slower by roughly a factor $t_{\mathrm{JR} 09} \simeq$ $1.3 \times t_{\mathrm{ABM} 11}$ followed by the grids of MSTW [22] with $t_{\mathrm{MSTW}} \simeq 2 \times t_{\mathrm{ABM} 11}$. The grids of NN21 [23] are last in this row with run times up by roughly a factor of $5, t_{\mathrm{NN} 21} \simeq$ $5 \times t_{\mathrm{ABM} 11}$. This is correlated with the enormous size of the latter grids of $\mathcal{O}(100)$ MByte for NN21 with $100 \mathrm{PDF}$ sets, as compared, e.g., to the size of $\mathcal{O}(5)$ MByte for ABM11 or to $\mathcal{O}(15)$ MByte for MSTW. Note that the NN21 grids with 1000 PDF sets have a size of about $\mathcal{O}(1)$ GByte and typical run times up by another order of magnitude, making PDF error computations even more inefficient.

\section{CONCLUSIONS}

We have presented the PDF set ABM11 that determines the parton content of the nucleon and measures $\alpha_{s}\left(M_{Z}\right)$ at NNLO accuracy in QCD. In order to achieve a description of our analysis that is as complete and as transparent as possible, we have provided an extensive discussion of all ingredients. This begins with the theoretical foundations that are most advanced and (almost) fully consistent at NNLO in QCD using well-defined renormalization schemes for all parameters involved. We have given detailed information on our treatment of the additional higher twist terms and nuclear corrections as well as on all data sets involved and on our fit ansatz. The results presented have been exposed to numerous consistency checks to test stability and the statistical quality.

We have found good agreement with our previous results in ABKM09, and the PDF sets of ABM11 are readily available with the LHAPDF library for precision phenomenology at the LHC and other hadron colliders. We have studied the differences with respect to other PDF groups using the code OPENQCDRAD for standardized precision comparison. In this way some of the differences with respect to other PDF sets could be explained. The observed differences in the gluon PDFs are clearly outstanding in this context. This has important implications for the Higgs boson searches at the LHC and requires urgently further 
NNLO(approx.) $\mu_{\mathbf{R}}=\mu_{\mathbf{F}}=\mathbf{E}_{\mathbf{T}}$

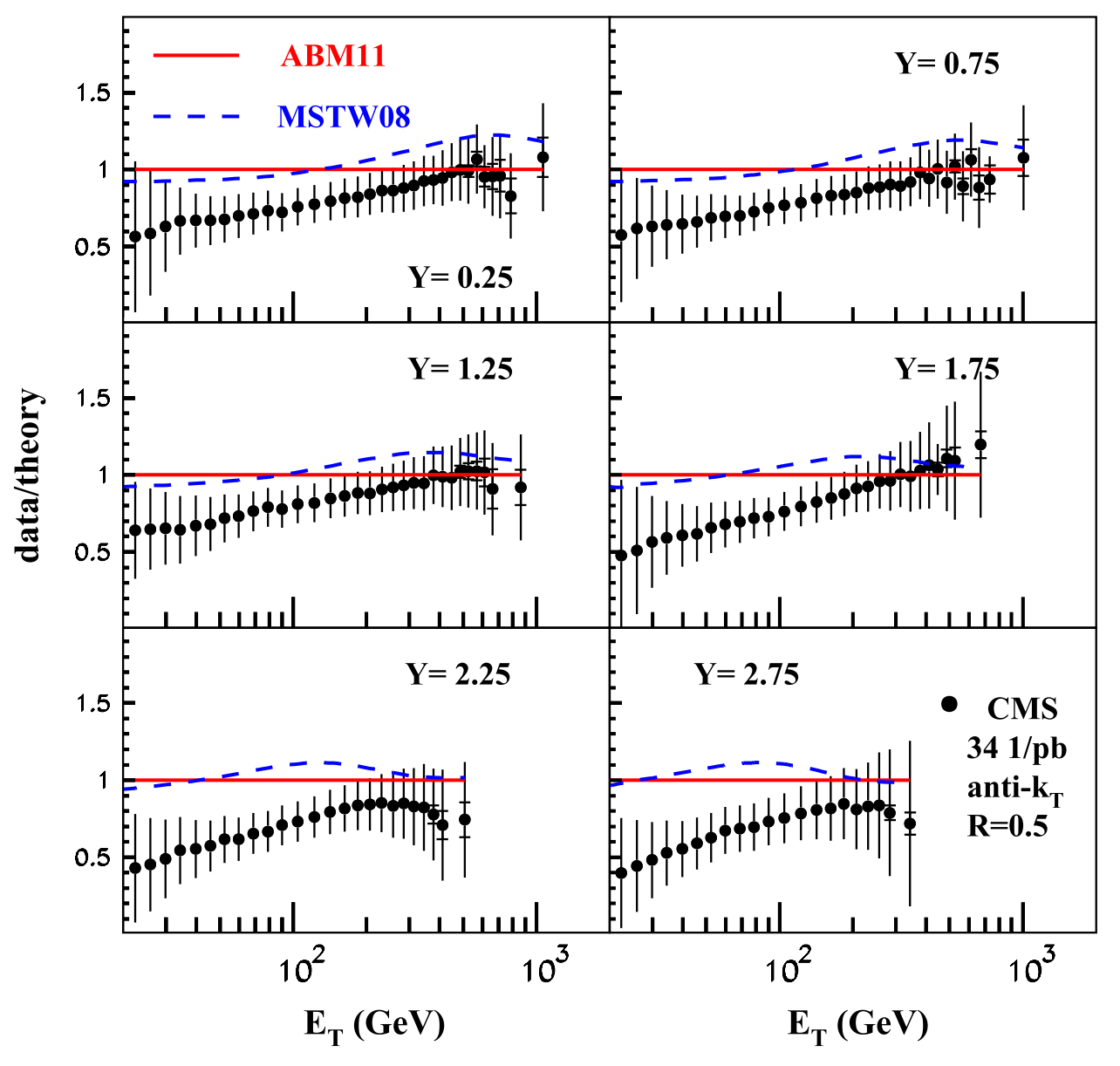

FIG. 29 (color online). Same as Fig. 26 for data of the CMS Collaboration [215].

studies. In our present fit, thanks to OPENQCDRAD, we have provided a framework in which such questions can be addressed in the future.

Despite the fact that various extractions of $\alpha_{s}\left(M_{Z}\right)$ have reached an impressive level of precision of $1 \%$, there exist still larger systematic differences that depend on both the particular observable under consideration as well as on the specific analysis carried out for a given observable (if compared to other analyses of the same observable). We have tried to analyze the status of the measurement of $\alpha_{s}\left(M_{Z}\right)$ from world DIS data and the effect of including other hard scattering data. At present we feel that not all data used in global analyses are of sufficient quality, considering the precision currently envisaged for $\alpha_{s}\left(M_{Z}\right)$.

The differences of the present analysis with respect to other groups have to be clarified in the future by performing dedicated mutual comparisons addressing the data sets used in the analyses, details of the theoretical description, as well as the data analysis. Here an important issue concerns the systematics of different data sets. In some cases the analyses show tensions and it is even known from the experimental analyses themselves that significant differences exist that are of systematic nature. It is a rather delicate matter how to deal with those data in rather refined analyses at the precision of the NNLO level in QCD. These effects are partly responsible for the different results presently obtained in PDF fits of various groups. In the future, this situation can be significantly improved by (i) the availability of NNLO QCD corrections to hard jet cross sections; (ii) hard multijet data from the LHC with wellcontrolled systematics and within a wider energy range than available at Tevatron; (iii) precise $W^{ \pm}, Z$, and Drell-Yan data from the LHC. Gradually, also other hard scattering cross sections will be understood to NNLO and allow one to constrain the PDFs much further.

It is therefore clear that the simplest way to reduce PDF uncertainties is not to discard nonglobal sets. Rather, it consists of very detailed studies of data sets, using the most complete theoretical descriptions, following D. Hilbert's request: "Wir müssen wissen - wir werden wissen!" [218]. Precision QCD analyses will always refer to compatible sets of precise data with a detailed account of the systematic errors and all known theoretical corrections, which allows one to measure all nonperturbative PDF parameters 
TABLE XXIII. Same as Table XXII for the LHC at $\sqrt{s}=8 \mathrm{TeV}$.

\begin{tabular}{|c|c|c|c|c|c|}
\hline$m_{t}$ & ABM11 & ABKM09 [16] & JR09 [20,21] & MSTW [22] & NN21 [23] \\
\hline 165 & $243.8_{-12.9-10.0}^{+4.6+10.0}$ & $247.4_{-12.9-12.7}^{+4.5+12.7}$ & $289.3_{-12.2-18.3}^{+3.9+18.3}$ & $298.1_{-16.0-7.4}^{+5.0+7.1}$ & $\begin{array}{l}307.5_{-14.7-7.6}^{+5.8+7.6} \\
\end{array}$ \\
\hline 166 & $236.2_{-12.6-9.7}^{+4.5+9.7}$ & $239.9_{-12.5-12.3}^{+4.3+12.3}$ & $279.5_{-10.6-17.9}^{+5.0+17.9}$ & $289.2_{-15.5-7.2}^{+4.9+7.0}$ & $298.3_{-14.2-7.4}^{+5.6+7.4}$ \\
\hline 167 & $229.0_{-12.2-9.4}^{+4.4+9.4}$ & $232.6_{-12.1-12.0}^{+4.2+12.0}$ & $271.2_{-10.2-17.4}^{+4.9+17.4}$ & $280.5_{-15.1-7.0}^{+4.7+6.8}$ & $289.2_{-13.7-7.2}^{+5.5+7.2}$ \\
\hline 168 & $222.0_{-11.8-9.2}^{+4.3+9.2}$ & $225.5_{-11.8-11.6}^{+4.1+11.6}$ & $263.2_{-10.0-17.1}^{+4.8+17.1}$ & $272.2_{-14.7-6.8}^{+4.6+6.6}$ & $280.6_{-13.3-7.0}^{+5.4+7.0}$ \\
\hline 169 & $215.2_{-11.5-8.9}^{+4.1+8.9}$ & $218.7_{-11.4-11.3}^{+4.0+11.3}$ & $255.4_{-9.6-16.6}^{+4.8+16.6}$ & $264.1_{-14.2-6.6}^{+4.6+6.4}$ & $272.2_{-12.8-6.8}^{+5.4+6.8}$ \\
\hline 170 & $208.7_{-11.1-8.7}^{+4.0+8.7}$ & $212.1_{-11.1-11.0}^{+3.9+11.0}$ & $248.0_{-9.3-16.2}^{+4.7+16.2}$ & $256.4_{-13.8-6.4}^{+4.5+6.2}$ & $264.1_{-12.4-6.6}^{+5.2+6.6}$ \\
\hline 171 & $202.4_{-10.8-8.4}^{+3.9+8.4}$ & $205.8_{-10.7-10.7}^{+3.8+10.7}$ & $240.7_{-8.9-15.8}^{+4.6+15.8}$ & $248.8_{-13.3-6.2}^{+4.4+6.1}$ & $256.3_{-11.9-6.5}^{+5.2+6.5}$ \\
\hline 172 & $196.4_{-10.5-8.2}^{+3.8+8.2}$ & $199.7_{-10.4-10.4}^{+3.7+10.4}$ & $233.7_{-8.7-15.5}^{+4.5+15.5}$ & $241.6_{-13.0-6.1}^{+4.2+5.9}$ & $248.8_{-11.6-6.3}^{+5.0+6.3}$ \\
\hline 173 & $190.5_{-10.2-8.0}^{+3.7+8.0}$ & $193.8_{-10.1-10.2}^{+3.6+10.2}$ & $227.0_{-8.4-15.1}^{+4.4+15.1}$ & $234.6_{-12.6-5.9}^{+4.1+5.8}$ & $241.5_{-11.2-6.1}^{+4.9+6.1}$ \\
\hline 174 & $184.8_{-9.9-7.8}^{+3.6+7.8}$ & $188.1_{-9.8-9.9}^{+3.5+9.9}$ & $220.4_{-8.1-14.7}^{+4.3+14.7}$ & $227.8_{-12.2-5.8}^{+4.0+5.6}$ & $234.5_{-10.8-6.0}^{+4.8+6.0}$ \\
\hline 175 & $179.3_{-9.6-7.6}^{+3.5+7.6}$ & $182.5_{-9.5-9.7}^{+3.4+9.7}$ & $214.0_{-7.9-14.4}^{+4.3+14.4}$ & $221.3_{-11.8-5.6}^{+4.0+5.5}$ & $227.9_{-10.6-5.8}^{+4.6+5.8}$ \\
\hline 176 & $174.1_{-9.3-7.4}^{+3.4+7.4}$ & $177.2_{-9.3-9.4}^{+3.3+9.4}$ & $207.9_{-7.6-14.2}^{+4.2+14.2}$ & $214.9_{-11.5-5.5}^{+3.9+5.4}$ & $221.3_{-10.3-5.7}^{+4.5+5.7}$ \\
\hline 177 & $168.9_{-9.0-7.2}^{+3.4+7.2}$ & $172.1_{-9.0-9.2}^{+3.2+9.2}$ & $202.0_{-7.4-13.8}^{+4.0+13.8}$ & $208.8_{-11.2-5.4}^{+3.7+5.2}$ & $215.0_{-10.1-5.6}^{+4.2+5.6}$ \\
\hline 178 & $164.0_{-8.8-7.0}^{+3.3+7.0}$ & $167.1_{-8.7-8.9}^{+3.2+8.9}$ & $196.2_{-7.1-13.5}^{+4.0+13.5}$ & $202.9_{-10.9-5.2}^{+3.7+5.1}$ & $208.9_{-9.8-5.4}^{+4.2+5.4}$ \\
\hline 179 & $159.3_{-8.5-6.8}^{+3.2+6.8}$ & $162.3_{-8.5-8.7}^{+3.1+8.7}$ & $190.9_{-7.0-13.1}^{+3.6+13.1}$ & $197.2_{-10.7-5.1}^{+3.5+5.0}$ & $203.1_{-9.7-5.3}^{+3.9+5.3}$ \\
\hline 180 & $154.7_{-8.3-6.6}^{+3.1+6.6}$ & $157.6_{-8.3-8.5}^{+3.0+8.5}$ & $185.6_{-6.8-12.8}^{+3.6+12.8}$ & $191.7_{-10.4-4.9}^{+3.5+4.8}$ & $197.3_{-9.3-5.1}^{+3.9+5.1}$ \\
\hline
\end{tabular}

TABLE XXIV. Same as Table XXII for the LHC at $\sqrt{s}=14 \mathrm{TeV}$.

\begin{tabular}{|c|c|c|c|c|c|}
\hline$m_{t}$ & ABM11 & ABKM09 [16] & JR09 [20,21] & MSTW [22] & NN21 [23] \\
\hline 165 & $995.2_{-43.6-28.5}^{+11.0+28.5}$ & $993.3_{-42.9-35.4}^{+10.7+35.4}$ & $1076.9_{-37.2-39.7}^{+8.3+39.7}$ & $1131.4_{-50.5-20.3}^{+11.6+18.4}$ & $1174.6_{-46.0-20.8}^{+14.0+20.8}$ \\
\hline 166 & $967.3_{-42.5-27.7}^{+10.7+27.7}$ & $965.6_{-41.9-34.5}^{+10.3+34.5}$ & $1043.1_{-31.6-38.9}^{+12.5+38.9}$ & $1100.2_{-49.1-19.9}^{+11.1+18.0}$ & $1141.9_{-44.3-20.3}^{+13.4+20.3}$ \\
\hline 167 & $940.2_{-41.3-27.0}^{+10.5+27.0}$ & $938.6_{-40.6-33.6}^{+10.2+33.6}$ & $1014.8_{-30.6-37.8}^{+12.3+37.8}$ & $1070.0_{-47.7-19.3}^{+10.8+17.6}$ & $1110.3_{-42.8-19.8}^{+13.7+19.8}$ \\
\hline 168 & $914.1_{-40.2-26.2}^{+10.2+26.2}$ & $912.7_{-39.6-32.8}^{+9.8+32.8}$ & $987.6_{-29.8-37.1}^{+12.2+37.1}$ & $1041.0_{-46.6-18.9}^{+10.6+17.1}$ & $1080.0_{-41.6-19.3}^{+13.1+19.3}$ \\
\hline 169 & $888.7_{-39.1-25.6}^{+9.9+25.6}$ & $887.4_{-38.5-32.1}^{+9.7+32.1}$ & $960.8_{-28.8-36.2}^{+12.4+36.2}$ & $1012.4_{-45.1-18.4}^{+10.8+16.7}$ & $1050.5_{-40.5-19.0}^{+13.0+19.0}$ \\
\hline 170 & $864.2_{-38.0-25.1}^{+9.7+25.1}$ & $863.1_{-37.5-31.3}^{+9.4+31.3}$ & $935.3_{-28.0-35.5}^{+12.1+35.5}$ & $985.3_{-44.0-17.9}^{+10.5+16.3}$ & $1022.0_{-39.4-18.5}^{+12.9+18.5}$ \\
\hline 171 & $840.5_{-37.0-24.4}^{+9.5+24.4}$ & $839.6_{-36.5-30.4}^{+9.1+30.4}$ & $910.0_{-26.8-34.6}^{+11.9+34.6}$ & $958.4_{-42.5-17.5}^{+10.4+15.9}$ & $994.1_{-37.8-18.0}^{+12.8+18.0}$ \\
\hline 172 & $817.6_{-36.0-23.8}^{+9.2+23.8}$ & $816.7_{-35.4-29.7}^{+8.9+29.7}$ & $886.1_{-26.5-33.9}^{+11.5+33.9}$ & $933.1_{-41.8-17.1}^{+9.9+15.6}$ & $967.7_{-37.0-17.6}^{+12.3+17.6}$ \\
\hline 173 & $795.3_{-35.0-23.3}^{+9.0+23.3}$ & $794.6_{-34.5-29.1}^{+8.8+29.1}$ & $862.7_{-25.6-33.1}^{+11.4+33.1}$ & $908.3_{-40.5-16.7}^{+9.8+15.2}$ & $941.8_{-35.8-17.1}^{+11.8+17.1}$ \\
\hline 174 & $773.8_{-34.1-22.7}^{+8.8+22.7}$ & $773.2_{-33.6-28.4}^{+8.6+28.4}$ & $840.0_{-24.7-32.4}^{+11.2+32.4}$ & $884.2_{-39.3-16.3}^{+9.6+14.9}$ & $916.7_{-34}^{+11}$ \\
\hline 175 & $752.9_{-33.2-22.2}^{+8.6+22.2}$ & $752.5_{-32.7-27.7}^{+8.4+27.7}$ & $817.9_{-23.9-31.9}^{+11.2+31.9}$ & $860.9_{-38.2-16.0}^{+9.5+14.6}$ & $893.0_{-34.1-16.3}^{+11.2+16.3}$ \\
\hline 176 & $732.7_{-32.3-21.7}^{+8.4+21.7}$ & $732.5_{-31.8-26.9}^{+8.1+26.9}$ & $796.4_{-23.0-31.5}^{+11.1+31.5}$ & $838.2_{-37.0-15.6}^{+9.5+14.3}$ & $869.9_{-33.8-16.0}^{+10.4+16.0}$ \\
\hline 177 & $713.2_{-31.5-21.2}^{+8.3+21.2}$ & $713.0_{-31.0-26.4}^{+8.0+26.4}$ & $775.8_{-22.6-30.7}^{+10.5+30.7}$ & $816.5_{-36.5-15.2}^{+8.8+14.0}$ & $847.0_{-32.8-15.6}^{+10.2+15.6}$ \\
\hline 178 & $694.3_{-30.7-20.7}^{+8.1+20.7}$ & $694.2_{-30.2-25.7}^{+7.8+25.7}$ & $755.6_{-21.6-30.0}^{+10.4+30.0}$ & $795.1_{-35.4-14.9}^{+8.8+13.7}$ & $824.9_{-31.8-15.2}^{+10.0+15.2}$ \\
\hline 179 & $675.9_{-29.9-20.2}^{+7.8+20.2}$ & $676.0_{-29.5-25.1}^{+7.6+25.1}$ & $736.9_{-21.5-29.3}^{+9.4+29.3}$ & $774.9_{-35.0-14.5}^{+8.2+13.3}$ & $804.0_{-31.4-14.9}^{+9.2+14.9}$ \\
\hline 180 & $\begin{array}{l}658.2_{-29.1-19.6}^{+7.6+19.6} \\
\end{array}$ & $658.3_{-28.7-24.5}^{+7.4+24.5}$ & $718.2_{-20.8-28.7}^{+9.4+28.7}$ & $754.9_{-33.9-14.2}^{+8.3+13.0}$ & $\begin{array}{l}783.1_{-30.6-14.6}^{+9.3+14.6} \\
\end{array}$ \\
\hline
\end{tabular}

and the strong coupling constant $\alpha_{s}\left(M_{Z}\right)$ as precisely as possible.

The QCD corrections are of vital importance to understand the production mechanism of new states as detailed as possible. In particular, the anticipated signal of a Higgs boson at a mass $m_{H} \sim 126 \mathrm{GeV}$ [219] has to be measured to highest precision. The aim of further PDF analyses has to consist in extracting both the gluon PDF and $\alpha_{s}\left(M_{Z}\right)$ at even higher statistical and systematic precision because the dominant Higgs production in $\mathrm{ggF}$ behaves 
TABLE XXV. Predicted cross sections for Higgs boson production in ggF with $m_{H}=120 \mathrm{GeV}$ at Tevatron $(\sqrt{s}=1.96 \mathrm{TeV})$ and at LHC $(\sqrt{s}=7 \mathrm{TeV})$ from NNLO variants of the ABKM09 fit [16] corresponding to Table VII. The uncertainty in brackets refers to the $1 \sigma$ standard deviation for the combined uncertainty on the PDFs and the value of $\alpha_{s}\left(M_{Z}\right)$. Values in bold correspond to the published result [26].

\begin{tabular}{lccccc}
\hline \hline$\sigma(H)[p b]$ & ABKM09 & D0 1-jet inc. & D0 dijet & CDF 1-jet inc. (cone) & CDF 1-jet inc. $\left(k_{T}\right)$ \\
\hline Tevatron(1.96) & $\mathbf{0 . 7 7 0}(\mathbf{5 0})$ & $0.859(29)$ & $0.833(27)$ & $0.815(25)$ & $0.842(25)$ \\
LHC(7) & $\mathbf{1 4 . 3 4}(\mathbf{4 1})$ & $14.68(29)$ & $14.69(27)$ & $14.11(28)$ & $14.44(27)$ \\
\hline \hline
\end{tabular}

TABLE XXVI. Covariance matrix for the PDF parameters in Table III, $\alpha_{s}, m_{c}$, and $m_{b}$.

\begin{tabular}{|c|c|c|c|c|c|c|c|c|c|c|c|c|c|c|}
\hline & $a_{u}$ & $b_{u}$ & $\gamma_{1, u}$ & $\gamma_{2, u}$ & $a_{d}$ & $b_{d}$ & $A_{\Delta}$ & $b_{\Delta}$ & $A_{u s}$ & $a_{u s}$ & $b_{u s}$ & $a_{g}$ & $b_{g}$ & $\gamma_{1, g}$ \\
\hline$a_{u}$ & 1.0000 & 0.9692 & 0.9787 & -0.7929 & 0.7194 & 0.5279 & -0.1460 & -0.1007 & 0.7481 & 0.6835 & -0.4236 & -0.2963 & 0.3391 & 0.3761 \\
\hline$b_{u}$ & & 1.0000 & 0.9396 & -0.7244 & 0.6792 & 0.4939 & -0.1146 & -0.1099 & 0.7404 & 0.6840 & -0.4146 & -0.3138 & 0.3464 & 0.3738 \\
\hline$\gamma_{1, u}$ & & & 1.0000 & -0.8940 & 0.6506 & 0.4646 & -0.1865 & -0.0539 & 0.6728 & 0.6093 & -0.4799 & -0.2755 & 0.3441 & 0.3717 \\
\hline$\gamma_{2, u}$ & & & & 1.0000 & -0.4102 & -0.2267 & 0.2357 & -0.0182 & -0.4075 & -0.3495 & 0.4543 & 0.1713 & -0.3156 & -0.3149 \\
\hline$a_{d}$ & & & & & 1.0000 & 0.8827 & -0.2155 & -0.1964 & 0.6875 & 0.6435 & -0.3030 & -0.3354 & 0.2635 & 0.3500 \\
\hline$b_{d}$ & & & & & & 1.0000 & -0.2462 & -0.0979 & 0.5359 & 0.5099 & -0.2957 & -0.3443 & 0.3157 & 0.3763 \\
\hline$A_{\Delta}$ & & & & & & & 1.0000 & -0.2068 & -0.0689 & -0.0698 & 0.2381 & -0.0168 & 0.0384 & 0.0453 \\
\hline$b_{\Delta}$ & & & & & & & & 1.0000 & 0.1015 & 0.1279 & -0.4146 & -0.0852 & -0.1185 & -0.0892 \\
\hline$A_{u s}$ & & & & & & & & & 1.0000 & 0.9884 & -0.4678 & -0.4679 & 0.1961 & 0.2504 \\
\hline$a_{u s}$ & & & & & & & & & & 1.0000 & -0.4520 & -0.5195 & 0.1982 & 0.2596 \\
\hline$b_{u s}$ & & & & & & & & & & & 1.0000 & 0.1436 & 0.0444 & -0.0180 \\
\hline$a_{g}$ & & & & & & & & & & & & 1.0000 & -0.6289 & -0.7662 \\
\hline$b_{g}$ & & & & & & & & & & & & & 1.0000 & 0.9392 \\
\hline$\gamma_{1, g}$ & & & & & & & & & & & & & & 1.0000 \\
\hline
\end{tabular}

as $\propto \alpha_{s}^{2} G\left(x, Q^{2}\right) \otimes G\left(x, Q^{2}\right)$ and it is well known that even the NNLO QCD corrections in ggF contribute a numerically significant portion to the total cross section. Also various other discoveries at hadron colliders may crucially depend on the thorough and precise understanding of the PDFs and QCD at high-energy scales.

\section{ACKNOWLEDGMENTS}

We thank J. Baglio, H. Böttcher, S. Dinter, A. Djouadi, V. Drach, P. Jimenez-Delgado, E. Reya, and R. Thorne for discussions. We are thankful to V. Drach for preparing the plot in Fig. 24, to R. Thorne for providing us with the corresponding NLO plots not contained in [159], and to $\mathrm{M}$. Zaro for computing the cross sections in Table XIX. We also gratefully acknowledge the support of M. Whalley to integrate the results of the ABM11 fit into the LHAPDF library [216,217].

J. B. acknowledges support from Technische Universität Dortmund. This work has been supported by Helmholtz Gemeinschaft under Contract No. VH-HA-101 (Alliance Physics at the Terascale), by the Deutsche Forschungsgemeinschaft in Sonderforschungsbereich/Transregio 9, and by the European Commission through Contract No. PITN-GA2010-264564 (LHCPhenoNet).

Note added in proof.-In the present analysis the comparison to NN21 has been carried out with the help of the grid NNPDF21_FFN3_100 in the LHAPDF in the LHAPDF repository dating from August 2, 2011. After publication of this article the grids of NN21 have been replaced and Figs. 11 and 22 have been updated with the grid NNPDF21_FFN3_100 now dating from February 17, 2012. With this new grid our conclusions remain unchanged. The NNLO CT10 PDFs have been recently included into the LHAPDF library [224].

\section{APPENDIX A: STATISTICS}

\section{Statistical procedures}

In our analysis we infer the vector of fitted parameters $\vec{\theta}$ from the experimental data minimizing the $\chi^{2}$ functional

$$
\chi^{2}(\vec{\theta})=\sum_{i, j=1}^{N}\left(f_{i}(\vec{\theta})-y_{i}\right) E_{i j}\left(f_{j}(\vec{\theta})-y_{j}\right),
$$

where $f_{i}(\vec{\theta})$ is the fitted model, $y_{i}$ are the measurements, and $E_{i j}$ is the measurement error matrix with the indexes $i$, $j$ running through all $N$ data points included into the fit. The error matrix $E_{i j}$ is the inverse of the covariance matrix $C_{i j}$. If the data are uncorrelated, the covariance matrix is diagonal and

$$
C_{i j}=\delta_{i j} \sigma_{i} \sigma_{j}
$$

where $\sigma_{i}$ are uncorrelated errors in the measurements $y_{i}$. If, in addition, the data are subject to the point-to-point 
TABLE XXVII. Covariance matrix for the PDF parameters in Table III, $\alpha_{s}, m_{c}$, and $m_{b}$.

\begin{tabular}{|c|c|c|c|c|c|c|c|c|c|c|c|c|c|}
\hline & $\alpha_{s}\left(\mu_{0}\right)$ & $\gamma_{1, \Delta}$ & $\gamma_{1, u s}$ & $\gamma_{1, d}$ & $\gamma_{2, d}$ & $A_{s}$ & $b_{s}$ & $a_{s}$ & $\gamma_{3, u}$ & $m_{c}\left(m_{c}\right)$ & $\gamma_{3, u s}$ & $m_{b}\left(m_{b}\right)$ & $a_{\Delta}$ \\
\hline$a_{u}$ & -0.0435 & 0.0000 & -0.8480 & 0.6008 & 0.1535 & -0.0034 & -0.0437 & -0.0355 & 0.8111 & 0.0796 & -0.4797 & 0.0044 & -0.1718 \\
\hline$b_{u}$ & -0.1251 & 0.0316 & -0.8375 & 0.5537 & 0.1806 & 0.0008 & -0.0345 & -0.0276 & 0.7001 & 0.0625 & -0.4889 & -0.0005 & -0.1452 \\
\hline$\gamma_{1, u}$ & -0.0849 & -0.0637 & -0.8133 & 0.5422 & 0.1667 & -0.0324 & -0.0671 & -0.0638 & 0.8948 & 0.0726 & -0.4033 & 0.0075 & -0.2028 \\
\hline$\gamma_{2, u}$ & 0.0920 & 0.1659 & 0.5760 & -0.3308 & -0.2276 & 0.0799 & 0.0966 & 0.1098 & -0.9749 & -0.0631 & 0.1728 & -0.0142 & 0.2353 \\
\hline$a_{d}$ & -0.0321 & -0.0137 & -0.7618 & 0.9630 & -0.1842 & 0.0007 & -0.0414 & -0.0167 & 0.4878 & 0.0227 & -0.4 & -0.0 & -0.2088 \\
\hline$b_{d}$ & -0.1666 & -0.1167 & -0.6060 & & -0.5969 & -0.0064 & -0.0249 & -0.0203 & & -0 & -0. & $-0 .($ & -0.2121 \\
\hline$A_{\Delta}$ & 0.0206 & 0.8718 & 0.1649 & -0.2544 & 0.1916 & -0.0232 & -0.0212 & -0.0294 & -0.2398 & 0.0202 & 0.0667 & 0.0034 & 0.9721 \\
\hline$b_{\Delta}$ & 0.0086 & -0.6291 & -0.1067 & -0.1834 & -0.1103 & 0.0594 & 0.0577 & 0.0711 & 0.0052 & -0.0063 & -0.1768 & -0.0083 & -0.0662 \\
\hline$A_{u s}$ & 0.0043 & -0.0481 & -0.8662 & 0.5862 & 0.0768 & -0.0341 & -0.0659 & -0.0493 & 0.4485 & 0.1559 & -0.8164 & -0.0008 & -0.0417 \\
\hline$a_{u s}$ & -0.0459 & -0.0650 & -0.8255 & 0.5493 & 0.0606 & -0.0119 & -0.0441 & -0.0255 & 0.3870 & 0.0940 & -0.8628 & -0.0055 & -0.0375 \\
\hline$b_{u s}$ & -0.0382 & 0.3783 & 0.7032 & -0.3288 & 0.1278 & -0.0734 & -0.0445 & -0.0807 & -0.4262 & -0.0100 & 0.3911 & 0.0040 & 0.1782 \\
\hline$a_{g}$ & 0.3785 & 0.0061 & 0.3050 & -0.3280 & 0.1338 & 0.0936 & 0.0718 & 0.1165 & -0.1744 & -0.0137 & 0.4886 & 0.0323 & -0.0360 \\
\hline$b_{g}$ & -0.6085 & 0.1017 & -0.0873 & 0.2827 & -0.2104 & -0.0543 & -0.0114 & -0.1223 & 0.2973 & 0.1560 & -0.1337 & 0.0141 & 0.0066 \\
\hline$\gamma_{1, g}$ & -0.4642 & 0.1021 & -0.1778 & 0.3605 & -0.1962 & -0.0708 & -0.0396 & -0.1230 & 0.3132 & 0.0425 & -0.1977 & 0.0071 & 0.0201 \\
\hline
\end{tabular}

TABLE XXVIII. Covariance matrix for the PDF parameters in Table III, $\alpha_{s}, m_{c}$, and $m_{b}$.

\begin{tabular}{|c|c|c|c|c|c|c|c|c|c|c|c|c|c|}
\hline & $\alpha_{s}\left(\mu_{0}\right)$ & $\gamma_{1, \Delta}$ & $\gamma_{1, u s}$ & $\gamma_{1, d}$ & $\gamma_{2, d}$ & $A_{s}$ & $b_{s}$ & $a_{s}$ & $\gamma_{3, u}$ & $m_{c}\left(m_{c}\right)$ & $\gamma_{3, u s}$ & $m_{b}\left(m_{b}\right)$ & $a_{\Delta}$ \\
\hline$\alpha_{s}\left(\mu_{0}\right)$ & 1.0000 & 0.0176 & -0.0394 & -0.0798 & 0.2357 & -0.0018 & -0.0982 & -0.0075 & -0.0291 & 0.1904 & 0.0676 & 0.0562 & 0.0136 \\
\hline$\gamma_{1, \Delta}$ & & 1.0000 & 0.1183 & -0.0802 & 0.2640 & -0.0427 & -0.0489 & -0.0550 & -0.1595 & 0.0193 & 0.0985 & 0.0069 & 0.7657 \\
\hline$\gamma_{1, u s}$ & & & 1.0000 & -0.6753 & -0.0493 & -0.0525 & 0.0158 & -0.0445 & -0.6039 & -0.0656 & 0.6590 & 0.0017 & 0.1487 \\
\hline$\gamma_{1, d}$ & & & & 1.0000 & -0.4041 & -0.0213 & -0.0513 & -0.0366 & 0.4145 & 0.0148 & -0.3931 & -0.0086 & -0.2284 \\
\hline$\gamma_{2, d}$ & & & & & 1.0000 & 0.0308 & -0.0016 & 0.0326 & 0.1801 & 0.0276 & -0.0510 & 0.0111 & 0.1212 \\
\hline$A_{s}$ & & & & & & 1.0000 & 0.8570 & 0.9749 & -0.0664 & -0.0206 & -0.4355 & 0.0017 & -0.0139 \\
\hline$b_{s}$ & & & & & & & 1.0000 & 0.8730 & -0.0894 & -0.0706 & -0.3708 & 0.0005 & -0.0127 \\
\hline$a_{s}$ & & & & & & & & 1.0000 & -0.0967 & -0.1234 & -0.4403 & -0.0050 & -0.0172 \\
\hline$\gamma_{3, u}$ & & & & & & & & & 1.0000 & 0.0674 & -0.2082 & 0.0153 & -0.2378 \\
\hline$m_{c}\left(m_{c}\right)$ & & & & & & & & & & 1.0000 & -0.0010 & 0.0505 & 0.0141 \\
\hline$\gamma_{3, u s}$ & & & & & & & & & & & 1.0000 & 0.0083 & 0.0276 \\
\hline$m_{b}\left(m_{b}\right)$ & & & & & & & & & & & & 1.0000 & 0.0006 \\
\hline$a_{\Delta}$ & & & & & & & & & & & & & 1.0000 \\
\hline
\end{tabular}

correlated systematic fluctuations, the off-diagonal terms appear in the covariance matrix as well. For counting experiments the systematic errors are commonly multiplicative. Therefore, the general form of the covariance matrix employed in our analysis reads

$$
C_{i j}=\eta_{i} \eta_{j} f_{i}(\vec{\theta}) f_{j}(\vec{\theta})+\delta_{i j} \sigma_{i} \sigma_{j}
$$

where $\eta_{i}$ is the relative correlated systematic error in the measurement $y_{i}$. The uncorrelated errors may stem both from the statistical and systematic uncertainties. In the later case they are combined with the statistical ones in quadrature to obtain $\sigma_{j}$. The estimator based on the functional of Eq. (A1) is statistically efficient and asymptotically unbiased in the limit of $N \rightarrow \infty$. In case of the analysis of correlated data, the fitted parameters may be biased [220]; however, for the definition of Eq. (A3) the estimator is nevertheless asymptotically unbiased [221]. The errors in the fitted parameters given in Table III and the correlations coefficients of Tables XXVI, XXVII, and XXVIII are propagated from the uncertainties in the data with account of the correlations in the latter and correspond to the standard statistical criterion $\Delta \chi^{2}=1$. The errors in the data normalization factors for the selected experiments, cf. Tables I and II, which were fitted simultaneously with other parameters, are calculated in the same way.

The detailed information on the experimental uncertainties in all data sets considered in the fit and listed in Sec. III are available from http://arxiv.org as an attachment to the arXiv version of our paper. This includes in particular the systematic errors in the SLAC data [222] discussed in Sec. III A and, likewise, the systematic errors in the NuTeV data and the corrections on the unmeasured phase space for the NuTeV and the CCFR data [223] discussed in Sec. III C.

\section{Parameter correlation matrix}

Here, we finally present the covariance matrix for the correlations of the fit parameters of ABM11 discussed in 
Sec. IV; cf. Table III and Eq. (4.10) for $m_{c}$ and $m_{b}$. The strong coupling $\alpha_{s}\left(M_{Z}\right)$ is given in Eq. (4.13), while in Tables XXVI, XXVII, and XXVIII we quote the correlations for $\alpha_{s}\left(\mu_{0}\right)$ with $\mu_{0}^{2}=1.5 \mathrm{GeV}^{2}$.
The correlations matrices for all other variants of the PDF fit discussed in the paper, e.g., those fits in Sec. VD including Tevatron jet data, are omitted for brevity. However, they are available from the authors upon request.
[1] S. Alekhin, Phys. Lett. B 519, 57 (2001).

[2] S. Moch, J. Vermaseren, and A. Vogt, Nucl. Phys. B688, 101 (2004).

[3] A. Vogt, S. Moch, and J. Vermaseren, Nucl. Phys. B691, 129 (2004).

[4] W. van Neerven and E. Zijlstra, Phys. Lett. B 272, 127 (1991).

[5] E. Zijlstra and W. van Neerven, Phys. Lett. B 273, 476 (1991).

[6] E. Zijlstra and W. van Neerven, Nucl. Phys. B383, 525 (1992).

[7] E. Zijlstra and W. van Neerven, Phys. Lett. B 297, 377 (1992).

[8] S. Moch and J. Vermaseren, Nucl. Phys. B573, 853 (2000).

[9] S. Moch, J. Vermaseren, and A. Vogt, Phys. Lett. B 606, 123 (2005).

[10] J. Vermaseren, A. Vogt, and S. Moch, Nucl. Phys. B724, 3 (2005).

[11] R. Hamberg, W. van Neerven, and T. Matsuura, Nucl. Phys. B359, 343 (1991).

[12] R. V. Harlander and W. B. Kilgore, Phys. Rev. Lett. 88, 201801 (2002).

[13] C. Anastasiou, L. J. Dixon, K. Melnikov, and F. Petriello, Phys. Rev. Lett. 91, 182002 (2003).

[14] C. Anastasiou, L. J. Dixon, K. Melnikov, and F. Petriello, Phys. Rev. D 69, 094008 (2004).

[15] S. Catani, L. Cieri, G. Ferrera, D. de Florian, and M. Grazzini, Phys. Rev. Lett. 103, 082001 (2009).

[16] S. Alekhin, J. Blümlein, S. Klein, and S. Moch, Phys. Rev. D 81, 014032 (2010).

[17] S. Alekhin, J. Blümlein, and S. Moch, Proc. Sci., DIS2010 (2010) 021

[18] F. Aaron et al. (H1 and ZEUS Collaboration), J. High Energy Phys. 01 (2010) 109.

[19] HERAPDF, https://www.desy.de/h1zeus/combined_results/index.php?do=proton_structure.

[20] P. Jimenez-Delgado and E. Reya, Phys. Rev. D 79, 074023 (2009).

[21] P. Jimenez-Delgado and E. Reya, Phys. Rev. D 80, 114011 (2009).

[22] A. Martin, W. Stirling, R. Thorne, and G. Watt, Eur. Phys. J. C 63, 189 (2009).

[23] R. D. Ball, V. Bertone, F. Cerutti, L. D. Debbio, S. Forte, A. Guffanti, J. I. Latorre, J. Rojo, and M. Ubiali, Nucl. Phys. B855, 153 (2012).

[24] H.-L. Lai, M. Guzzi, J. Huston, Z. Li, P. M. Nadolsky, J. Pumplin, and C.-P. Yuan, Phys. Rev. D 82, 074024 (2010).

[25] J. Baglio and A. Djouadi, J. High Energy Phys. 10 (2010) 064.

[26] S. Alekhin, J. Blümlein, P. Jimenez-Delgado, S. Moch, and E. Reya, Phys. Lett. B 697, 127 (2011).
[27] S. Alekhin, J. Blümlein, and S. Moch, Eur. Phys. J. C 71, 1723 (2011).

[28] R. Thorne and G. Watt, J. High Energy Phys. 08 (2011) 100.

[29] G. Watt, J. High Energy Phys. 09 (2011) 069.

[30] R. D. Ball et al., Phys. Lett. B 704, 36 (2011).

[31] F. Aaron et al. (H1 Collaboration), Eur. Phys. J. C 71, 1579 (2011).

[32] S. Alekhin and S. Moch, Phys. Lett. B 699, 345 (2011).

[33] A. Vogt, Comput. Phys. Commun. 170, 65 (2005).

[34] G. P. Salam and J. Rojo, Comput. Phys. Commun. 180, 120 (2009).

[35] S. Moch, J. Vermaseren, and A. Vogt, Nucl. Phys. B813, 220 (2009)

[36] E. Laenen, S. Riemersma, J. Smith, and W. van Neerven, Nucl. Phys. B392, 162 (1993).

[37] T. Gottschalk, Phys. Rev. D 23, 56 (1981).

[38] M. Glück, S. Kretzer, and E. Reya, Phys. Lett. B 380, 171 (1996).

[39] E. Laenen and S. Moch, Phys. Rev. D 59, 034027 (1999).

[40] S. Alekhin and S. Moch, Phys. Lett. B 672, 166 (2009).

[41] N. Presti, H. Kawamura, S. Moch, and A. Vogt, Proc. Sci., DIS2010 (2010) 163.

[42] G. Corcella and A. D. Mitov, Nucl. Phys. B676, 346 (2004).

[43] M. Buza, Y. Matiounine, J. Smith, R. Migneron, and W. van Neerven, Nucl. Phys. B472, 611 (1996).

[44] M. Buza, Y. Matiounine, J. Smith, and W. van Neerven, Eur. Phys. J. C 1, 301 (1998).

[45] I. Bierenbaum, J. Blümlein, and S. Klein, Nucl. Phys. B780, 40 (2007).

[46] I. Bierenbaum, J. Blumlein, and S. Klein, Phys. Lett. B 672, 401 (2009).

[47] I. Bierenbaum, J. Blümlein, and S. Klein, Nucl. Phys. B820, 417 (2009).

[48] J. Ablinger, J. Blümlein, S. Klein, C. Schneider, and F. Wissbrock, Nucl. Phys. B844, 26 (2011).

[49] S. Alekhin and J. Blümlein, Phys. Lett. B 594, 299 (2004).

[50] J. Blümlein, A. Hasselhuhn, P. Kovacikova, and S. Moch, Phys. Lett. B 700, 294 (2011).

[51] S. Larin, T. van Ritbergen, and J. Vermaseren, Nucl. Phys. B438, 278 (1995).

[52] K. Chetyrkin, B. A. Kniehl, and M. Steinhauser, Phys. Rev. Lett. 79, 2184 (1997).

[53] OPENQCDRAD, http://www-zeuthen.desy.de/ alekhin/ OPENQCDRAD.

[54] A. Martin, R. Roberts, W. Stirling, and R. Thorne, Eur. Phys. J. C 39, 155 (2005).

[55] S. Moch, J. Vermaseren, and A. Vogt, Nucl. Phys. B621, 413 (2002) 
[56] A. Vogt, S. Moch, and J. Vermaseren, Acta Phys. Pol. B 37, 683 (2006).

[57] H. Georgi and H. Politzer, Phys. Rev. D 14, 1829 (1976).

[58] O. Nachtmann, Nucl. Phys. B63, 237 (1973).

[59] M. Virchaux and A. Milsztajn, Phys. Lett. B 274, 221 (1992).

[60] J. Blümlein, H. Böttcher, and A. Guffanti, Nucl. Phys. B774, 182 (2007).

[61] J. Blümlein and H. Böttcher, Phys. Lett. B 662, 336 (2008).

[62] L. Whitlow, S. Rock, A. Bodek, E. Riordan, and S. Dasu, Phys. Lett. B 250, 193 (1990).

[63] E. V. Shuryak and A. Vainshtein, Nucl. Phys. B199, 451 (1982).

[64] S. Alekhin, Phys. Rev. D 63, 094022 (2001).

[65] V. Braun, A. Manashov, and J. Rohrwild, Nucl. Phys. B826, 235 (2010).

[66] S. Alekhin, S. A. Kulagin, and R. Petti, AIP Conf. Proc. 967, 215 (2007).

[67] S. Alekhin, S. A. Kulagin, and S. Liuti, Phys. Rev. D 69 , 114009 (2004).

[68] S. Alekhin, S. A. Kulagin, and R. Petti, Phys. Lett. B 675, 433 (2009).

[69] S. Alekhin, S. A. Kulagin, and R. Petti, arXiv:0810.4893.

[70] S. A. Kulagin and R. Petti, Nucl. Phys. A765, 126 (2006).

[71] J. Arrington, J. Rubin, and W. Melnitchouk, Phys. Rev. Lett. 108, 252001 (2012).

[72] S. Kulagin and R. Petti, Phys. Rev. C 82, 054614 (2010).

[73] M. Lacombe, B. Loiseau, J. M. Richard, R. Vinh Mau, J. Côté, P. Pirès, and R. de Tourreil, Phys. Rev. C 21, 861 (1980).

[74] R. Machleidt, K. Holinde, and C. Elster, Phys. Rep. 149, 1 (1987).

[75] S. A. Kulagin and R. Petti, Phys. Rev. D 76, 094023 (2007).

[76] D. Alde et al., Phys. Rev. Lett. 64, 2479 (1990).

[77] A. Bodek et al., Phys. Rev. D 20, 1471 (1979).

[78] W. Atwood, E. D. Bloom, R. L. A. Cottrell, H. DeStaebler, M. Mestayer, C. Y. Prescott, L. S. Rochester, S. Stein, R. E. Taylor, and D. Trines, Phys. Lett. 64B, 479 (1976).

[79] M. D. Mestayer, W.B. Atwood, E. D. Bloom, R. L. A. Cottrell, H. DeStaebler, C. Y. Prescott, L.S. Rochester, S. Stein, R.E. Taylor, D. Trines, Phys. Rev. D 27, 285 (1983).

[80] J. Gomez et al., Phys. Rev. D 49, 4348 (1994).

[81] S. Dasu et al., Phys. Rev. D 49, 5641 (1994).

[82] A. Benvenuti et al. (BCDMS Collaboration), Phys. Lett. B 223, 485 (1989).

[83] A. Benvenuti et al. (BCDMS Collaboration), Phys. Lett. B 237, 592 (1990).

[84] M. Arneodo et al. (NMC Collaboration), Nucl. Phys. B483, 3 (1997).

[85] A. Arbuzov, D. Y. Bardin, J. Blümlein, L. Kalinovskaya, and T. Riemann, Comput. Phys. Commun. 94, 128 (1996).

[86] C. Adloff et al. (H1 Collaboration), Eur. Phys. J. C 21, 33 (2001).

[87] S. Chekanov et al. (ZEUS Collaboration), Eur. Phys. J. C 21, 443 (2001).

[88] M. Arneodo et al. (NMC Collaboration), Phys. Lett. B 364, 107 (1995)

[89] L. Whitlow, Ph.D. thesis, Stanford University, 1990.

[90] G. Moreno et al., Phys. Rev. D 43, 2815 (1991).
[91] R. Towell et al. (FNAL E866/NuSea Collaboration), Phys. Rev. D 64, 052002 (2001).

[92] J. Webb et al. (NuSea Collaboration), arXiv:hep-ex/ 0302019.

[93] S. Alekhin, K. Melnikov, and F. Petriello, Phys. Rev. D 74, 054033 (2006).

[94] A. Bazarko et al. (CCFR Collaboration), Z. Phys. C 65 , 189 (1995).

[95] M. Goncharov et al. (NuTeV Collaboration), Phys. Rev. D 64, 112006 (2001).

[96] D. A. Mason, Ph.D. thesis, University of Oregon, 2006.

[97] S. Alekhin, J. Blümlein, H. Böttcher, and S. Moch, arXiv:1104.0469.

[98] K. Nakamura et al. (Particle Data Group), J. Phys. G 37, 075021 (2010).

[99] S. Malace et al. (Jefferson Lab E00-115 Collaboration), Phys. Rev. C 80, 035207 (2009).

[100] R. D. Ball, V. Bertone, F. Cerutti, L. Del Debbio, S. Forte, A. Guffanti, J. I. Latorre, J. Rojo, and M. Ubiali, Nucl. Phys. B849, 296 (2011).

[101] J. Sanchez Guillen, J. Miramontes, M. Miramontes, G. Parente, and O. Sampayo, Nucl. Phys. B353, 337 (1991).

[102] S. Alekhin, J. Blümlein, and S. Moch, arXiv:1105.5349.

[103] J. Blümlein and H. Böttcher, arXiv:1207.3170.

[104] J. Blümlein, S. Riemersma, W. van Neerven, and A. Vogt, Nucl. Phys. B, Proc. Suppl. 51, 97 (1996).

[105] M. Arneodo et al. (New Muon Collaboration), Phys. Lett. B 309, 222 (1993).

[106] R. D. Ball,V. Bertone,L. Del Debbio,S. Forte,A. Guffanti, J. I. Latorre,S. Lionetti,J. Rojo,M. Ubiali , Phys. Lett. B 707, 66 (2012).

[107] A. D. Martin, W. J. Stirling, R. S. Thorne, and G. Watt, Eur. Phys. J. C 64, 653 (2009).

[108] A. Abulencia et al. (CDF Collaboration), Phys. Rev. D 75, 092006 (2007).

[109] T. Aaltonen et al. (CDF Collaboration), Phys. Rev. D 78, 052006 (2008).

[110] V. Abazov et al. (D0 Collaboration), Phys. Rev. Lett. 101, 062001 (2008).

[111] V. Abazov et al. (D0 Collaboration), Phys. Lett. B 693, 531 (2010).

[112] N. Kidonakis and J. Owens, Phys. Rev. D 63, 054019 (2001).

[113] T. Affolder et al. (CDF Collaboration), Phys. Rev. Lett. 88, 042001 (2002).

[114] V. M. Abazov et al. (D0 Collaboration), Phys. Rev. D 80, 111107 (2009).

[115] K. Rabbertz, CERN Report No. CMS-NOTE-2011-004, 2011 (unpublished).

[116] D. Bandurin (D0 and CDF Collaboration), arXiv:1112.0051.

[117] R. Frederix, S. Frixione, K. Melnikov, and G. Zanderighi, J. High Energy Phys. 11 (2010) 050.

[118] B. Shaikhatdenov, A. Kotikov, V. Krivokhizhin, and G. Parente, Phys. Rev. D 81, 034008 (2010).

[119] A. Benvenuti et al. (BCDMS Collaboration), Phys. Lett. B 223, 490 (1989).

[120] A. Benvenuti et al. (BCDMS Collaboration), Phys. Lett. B 237, 599 (1990).

[121] L. Whitlow, E. Riordan, S. Dasu, S. Rock, and A. Bodek, Phys. Lett. B 282, 475 (1992). 
[122] M. Glück, E. Reya, and C. Schuck, Nucl. Phys. B754, 178 (2006).

[123] P. Nadolsky, News from CTEQ-TEA PDF analysis, 2011, http://indico.desy.de/conferenceDisplay.py?confId=4211.

[124] T. Gehrmann, M. Jaquier, and G. Luisoni, Eur. Phys. J. C 67, 57 (2010).

[125] R. Abbate, M. Fickinger, A. H. Hoang, V. Mateu, and I. W. Stewart, Phys. Rev. D 83, 074021 (2011).

[126] G. Dissertori, A. Gehrmann-DeRidder, T. Gehrmann, E. W. N. Glover, G. Heinrich, and H. Stenzel, Phys. Rev. Lett. 104, 072002 (2010).

[127] P. A. Baikov, K. G. Chetyrkin, and J. H. Kühn, Phys. Rev. Lett. 101, 012002 (2008).

[128] P. Baikov, K. Chetyrkin, J. Kühn, and J. Rittinger, Phys. Rev. Lett. 108, 222003 (2012).

[129] S. Bethke et al., arXiv:1110.0016.

[130] D. Boito, O. Catà, M. Golterman, M. Jamin, K. Maltman, J. Osborne, and S. Peris, Phys. Rev. D 84, 113006 (2011).

[131] S. Aoki et al. (PACS-CS Collaboration), J. High Energy Phys. 10 (2009) 053.

[132] C. McNeile, C. T.H. Davies, E. Follana, K. Hornbostel, and G. P. Lepage, Phys. Rev. D 82, 034512 (2010).

[133] B. Blossier, Ph. Boucaud, M. Brinet, F. De Soto, X. Du, V. Morenas, O. Pène, K. Petrov, J. Rodríguez-Quintero, Phys. Rev. Lett. 108, 262002 (2012).

[134] S. Bethke, Eur. Phys. J. C 64, 689 (2009).

[135] P. A. Baikov and K. G. Chetyrkin, Nucl. Phys. B, Proc. Suppl. 160, 76 (2006).

[136] V. Velizhanin, Nucl. Phys. B860, 288 (2012).

[137] S. Lionetti, R. D. Ball, V. Bertone, F. Cerutti, L. Del Debbio, S. Forte, A. Guffanti, J. I. Latorre, J. Rojo, and M. Ubiali, Phys. Lett. B 701, 346 (2011).

[138] M. Arneodo et al. (New Muon Collaboration), Nucl. Phys. B487, 3 (1997).

[139] S. Chekanov et al. (ZEUS Collaboration), Eur. Phys. J. C 61, 223 (2009).

[140] S. Chekanov et al. (ZEUS Collaboration), Eur. Phys. J. C 62, 625 (2009).

[141] J. Breitweg et al. (ZEUS Collaboration), Eur. Phys. J. C 12, 35 (2000).

[142] S. Chekanov et al. (ZEUS Collaboration), Phys. Rev. D 69, 012004 (2004).

[143] S. Chekanov et al. (ZEUS Collaboration), Eur. Phys. J. C 63, 171 (2009).

[144] S. Chekanov et al. (ZEUS Collaboration), Eur. Phys. J. C 65, 65 (2010).

[145] J. C. Webb, arXiv:hep-ex/0301031.

[146] T. Aaltonen et al. (CDF Collaboration), Phys. Rev. Lett. 102, 181801 (2009).

[147] T. A. Aaltonen et al. (CDF Collaboration), Phys. Lett. B 692, 232 (2010).

[148] V. Abazov et al. (D0 Collaboration), Phys. Rev. D 76, 012003 (2007).

[149] F. D. Aaron et al. (H1 Collaboration), Eur. Phys. J. C 65, 363 (2010).

[150] S. Chekanov et al. (ZEUS Collaboration), Report No. ZEUS-prel-10-002.

[151] G. Grindhammer, AIP Conf. Proc. 1441, 225 (2012).

[152] R. Kogler, Nucl. Phys. B, Proc. Suppl. 222, 81 (2012).

[153] R. Thorne (private communication).
[154] M. R. Adams et al. (E665 Collaboration), Phys. Rev. D 54, 3006 (1996).

[155] M. Tzanov et al. (NuTeV Collaboration), Phys. Rev. D 74, 012008 (2006).

[156] G. Onengut et al. (CHORUS Collaboration), Phys. Lett. B 632, 65 (2006).

[157] E. M. Lobodzinska, arXiv:hep-ph/0311180.

[158] C. Adloff et al. (H1 Collaboration), Eur. Phys. J. C 30, 1 (2003).

[159] C. Adloff et al. (H1 Collaboration), Eur. Phys. J. C 19, 269 (2001).

[160] J. Breitweg et al. (ZEUS Collaboration), Eur. Phys. J. C 7, 609 (1999).

[161] S. Chekanov et al. (ZEUS Collaboration), Eur. Phys. J. C 28, 175 (2003).

[162] S. Chekanov et al. (ZEUS Collaboration), Phys. Rev. D 70, 052001 (2004).

[163] S. Chekanov et al. (ZEUS Collaboration), Eur. Phys. J. C 32, 1 (2003).

[164] C. Adloff et al. (H1 Collaboration), Z. Phys. C 72, 593 (1996).

[165] C. Adloff et al. (H1 Collaboration), Phys. Lett. B 528, 199 (2002).

[166] A. Aktas et al. (H1 Collaboration), Eur. Phys. J. C 45, 23 (2006).

[167] A. Aktas et al. (H1 Collaboration), Eur. Phys. J. Special Topics 40, 349 (2005).

[168] A. Aktas et al. (H1 Collaboration), Phys. Lett. B 653, 134 (2007).

[169] S. Chekanov et al. (ZEUS Collaboration), Phys. Lett. B 547, 164 (2002).

[170] S. Chekanov et al. (ZEUS Collaboration), Nucl. Phys. B765, 1 (2007).

[171] V. M. Abazov et al. (D0 Collaboration), Phys. Rev. D 77, 011106 (2008).

[172] D. Acosta et al. (CDF Collaboration), Phys. Rev. D 71, 051104 (2005).

[173] J. Han et al. (CDF Collaboration), public note, 2008, www-cdf.fnal.gov/physics/ewk/2008/dszdy/.

[174] R. Sommer (private communication).

[175] S. Alekhin and S. Moch, arXiv:1107.0469.

[176] F. Aaron et al. (H1 Collaboration), Eur. Phys. J. C 71, 1769 (2011).

[177] B. Harris and J. Smith, Nucl. Phys. B452, 109 (1995).

[178] H1 and ZEUS Collaboration, Report No. H1prelim-10143 and No. ZEUS-prel-10-019, edited by A. CooperSarkar, S. Glazov, K. Lipka, R. Placakyte, and V. Radescu.

[179] D. Pleiter et al. (QCDSF/UKQCD Collaboration), Proc. Sci., LATTICE2010 (2010) 153.

[180] Y. Aoki, T. Blum, H.-W. Lin, S. Ohta, S. Sasaki, R. Tweedie, J. Zanotti, and T. Yamazaki, Phys. Rev. D 82, 014501 (2010).

[181] J. Bratt et al. (LHPC Collaboration), Phys. Rev. D 82, 094502 (2010).

[182] C. Alexandrou, J. Carbonell, M. Constantinou, P. A. Harraud, P. Guichon, K. Jansen, C. Kallidonis, T. Korzec, and M. Papinutto, Phys. Rev. D 83, 114513 (2011).

[183] S. Dinter et al., Proc. Sci., LATTICE2010 (2010) 135.

[184] S. Dinter, C. Alexandrou, M. Constantinou, V. Drach, K. Jansen, and D. B. Renner, Phys. Lett. B 704, 89 (2011).

[185] G. S. Bali et al., arXiv:1207.1110. 
[186] C. Anastasiou and K. Melnikov, Nucl. Phys. B646, 220 (2002).

[187] V. Ravindran, J. Smith, and W. van Neerven, Nucl. Phys. B665, 325 (2003).

[188] V. Ravindran, J. Smith, and W. van Neerven, Nucl. Phys. B704, 332 (2005).

[189] P. Bolzoni, F. Maltoni, S. Moch, and M. Zaro, Phys. Rev. Lett. 105, 011801 (2010).

[190] P. Bolzoni, F. Maltoni, S. Moch, and M. Zaro, Phys. Rev. D 85, 035002 (2012).

[191] O. Brein, A. Djouadi, and R. Harlander, Phys. Lett. B 579, 149 (2004).

[192] U. Langenfeld, S. Moch, and P. Uwer, Phys. Rev. D 80, 054009 (2009).

[193] M. Aliev et al., Comput. Phys. Commun. 182, 1034 (2011).

[194] S. Catani, G. Ferrera, and M. Grazzini, J. High Energy Phys. 05 (2010) 006.

[195] M. Glück, P. Jimenez-Delgado, E. Reya, and C. Schuck, Phys. Lett. B 664, 133 (2008).

[196] G. Aad et al. (ATLAS Collaboration), Phys. Lett. B 701, 31 (2011).

[197] S. Chatrchyan et al. (CMS Collaboration), J. High Energy Phys. 04 (2011) 050.

[198] R. Gavin, Y. Li, F. Petriello, and S. Quackenbush, Comput. Phys. Commun. 182, 2388 (2011).

[199] Y. Amhis (for the LHCb Collaboration), arXiv:1202.0654.

[200] S. Moch and A. Vogt, Phys. Lett. B 631, 48 (2005).

[201] G. Ferrera, M. Grazzini, and F. Tramontano, Phys. Rev. Lett. 107, 152003 (2011).

[202] B. A. Kniehl, Phys. Rev. D 42, 2253 (1990).

[203] O. Brein, R. Harlander, M. Wiesemann, and T. Zirke, Eur. Phys. J. C 72, 1868 (2012).

[204] V. M. Abazov et al. (D0 Collaboration), Phys. Lett. B 703, 422 (2011).
[205] A. Gehrmann-De Ridder, E. Glover, and J. Pires, J. High Energy Phys. 02 (2012) 141.

[206] P. Bolzoni, G. Somogyi, and Z. Trocsanyi, J. High Energy Phys. 01 (2011) 059.

[207] Z. Nagy, Phys. Rev. Lett. 88, 122003 (2002).

[208] Z. Nagy, Phys. Rev. D 68, 094002 (2003).

[209] T. Kluge, K. Rabbertz, and M. Wobisch, arXiv:hep-ph/ 0609285.

[210] M. Wobisch, D. Britzger, T. Kluge, K. Rabbertz, and F. Stober, arXiv:1109.1310.

[211] B. Malaescu and P. Starovoitov, Eur. Phys. J. C72, 2041 (2012).

[212] M. Wobisch, Nucl. Phys. B, Proc. Suppl. 222, 204 (2012).

[213] V. M. Abazov et al. (D0 Collaboration), Phys. Lett. B 704, 434 (2011).

[214] G. Aad et al. (ATLAS Collaboration), Phys. Rev. D 86, 014022 (2012).

[215] S. Chatrchyan et al. (CMS Collaboration), Phys. Rev. Lett. 107, 132001 (2011).

[216] M. Whalley, D. Bourilkov, and R. Group, arXiv:hep-ph/ 0508110 .

[217] LHAPDF, http://hepforge.cedar.ac.uk/lhapdf/.

[218] D. Hilbert, Address to the Society of German Scientists and Physicians, at Königsberg, 1930.

[219] ATLAS and CMS Collaborations, Talks at CERN on the update of the Higgs-boson search, 2012, http://indico .cern.ch/conferenceDisplay.py?confId=197461.

[220] G. D’Agostini, Nucl. Instrum. Methods Phys. Res., Sect. A 346, 306 (1994).

[221] S. Alekhin, arXiv:hep-ex/0005042.

[222] L. Whitlow (private communication).

[223] D. Mason (private communication).

[224] P. Nadolsky, J. Gao, M. Guzzi, J. Huston, H.-L. Lai, Z. Li, J. Pumplin, D. Stump, and C.-P. Yuan, arXiv:1206.3321. 